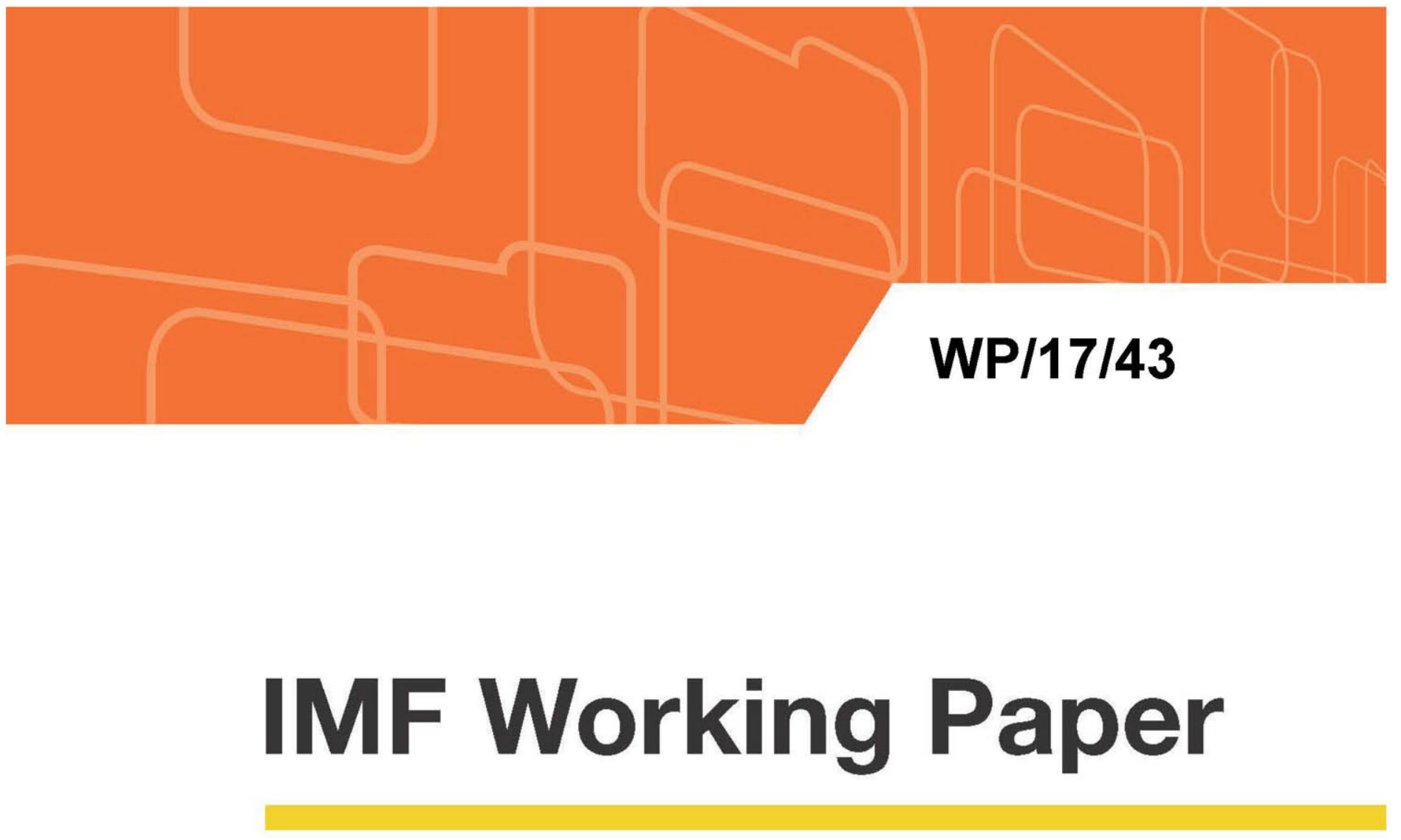

\title{
Launching Export Accelerations in Latin America and the World
}

by Valerie Cerra and Martha Tesfaye Woldemichael

IMF Working Papers describe research in progress by the author(s) and are published to elicit comments and to encourage debate. The views expressed in IMF Working Papers are those of the author(s) and do not necessarily represent the views of the IMF, its Executive Board, or IMF management. 


\section{WP/17/43}

\section{IMF Working Paper}

\section{Launching Export Accelerations in Latin America and the} World

by Valerie Cerra and Martha Tesfaye Woldemichael

IMF Working Papers describe research in progress by the author(s) and are published to elicit comments and to encourage debate. The views expressed in IMF Working Papers are those of the author(s) and do not necessarily represent the views of the IMF, its Executive Board, or IMF management.

$$
\text { I N T ER N A T I O N A L M O NETAR Y FU N D }
$$




\title{
IMF Working Paper
}

Western Hemisphere Department

\author{
Launching Export Accelerations in Latin America and the World ${ }^{1}$ \\ Prepared by Valerie Cerra and Martha Tesfaye Woldemichael
}

March 2017

IMF Working Papers describe research in progress by the author(s) and are published to elicit comments and to encourage debate. The views expressed in IMF Working Papers are those of the author(s) and do not necessarily represent the views of the IMF, its Executive Board, or IMF management.

\begin{abstract}
This paper investigates the determinants of sustained accelerations in goods and services exports. Strong predictors of export takeoffs include domestic and structural indicators such as lower macroeconomic uncertainty, improved quality of institutions, a depreciated exchange rate, and agricultural reforms. Lower tariffs, participation in global value chains and diversification also contribute to initiating export accelerations. The paper also finds heterogeneity, with somewhat different triggers for Latin America and the Caribbean, as well as for goods and services. Finally, despite the lack of a robust effect on output, export surges tend to be associated with lower post-acceleration unemployment and income inequality.

JEL Classification Numbers: F13, F14, F43, I3, O1, O54

Keywords: export accelerations, trade, growth, unemployment, inequality, probit, synthetic control method, Latin America and the Caribbean; globalization

Authors’ E-Mail Addresses: VCerra@imf.org; Martha.T.Woldemichael@gmail.com
\end{abstract}

\footnotetext{
${ }^{1}$ We would like to thank Alejandro Werner, Kim Beaton, Metodij Hadzi-Vaskov, Tidiane Kinda, Andras Komaromi, Pierre Mandon, Natalija Novta, Martha Denisse Pierola, and WHD seminar participants for helpful comments. This paper was prepared as a background study for the Western Hemisphere Department's Cluster Report on Trade Integration in Latin America and the Caribbean.
} 


\section{Contents}

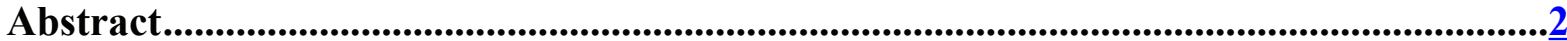

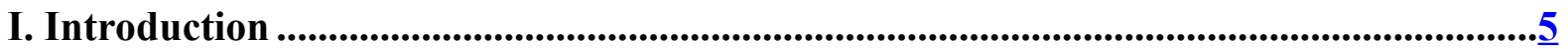

II. Identification of Export Acceleration Dates ............................................................

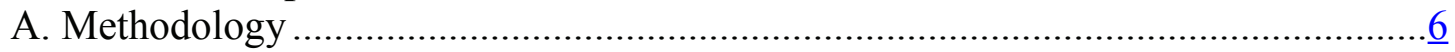

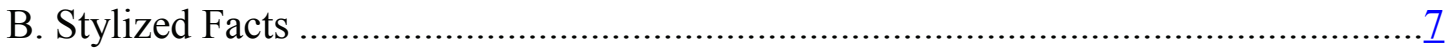

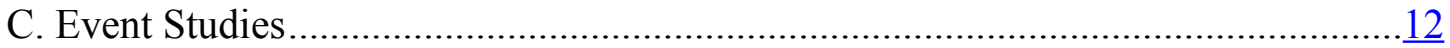

III. Correlates of Export Accelerations ...............................................................................15

A. EconometricModel ............................................................................ 15

Domestic Macroeconomic and Governance Indicators .............................16

Real Exchange Rate and Diversification ............................................ 17

Trade Policy and Product Market Reforms .......................................... 18

Financial Liberalization ................................................................. 18

Globalization and GVC Participation .................................................. 19

B. Baseline Results ....................................................................................

C. Robustness and Sensitivity Checks ........................................................ $\frac{30}{30}$

Choice of Parameters ................................................................................

Alternative Estimation Methods ...........................................................

Disaggregating Exports..................................................................

Splitting the Period of Study ..............................................................

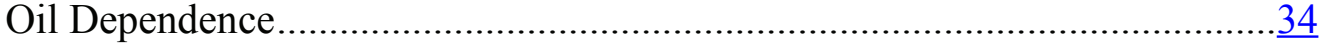

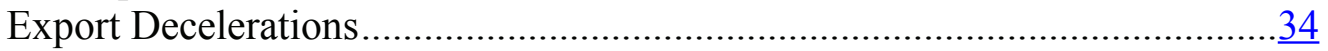

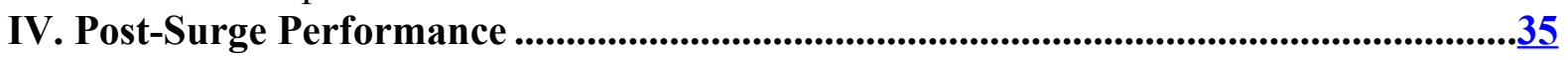

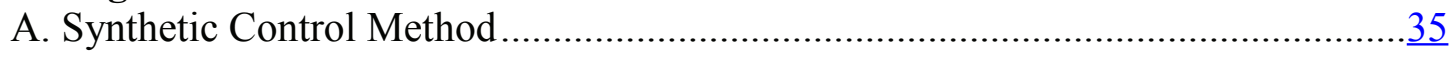

B. Data and Case Study Selection................................................................. $\frac{38}{39}$

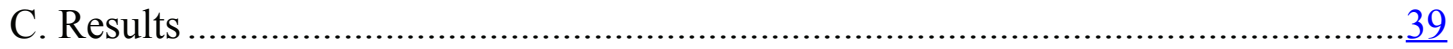

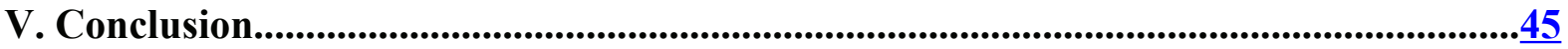

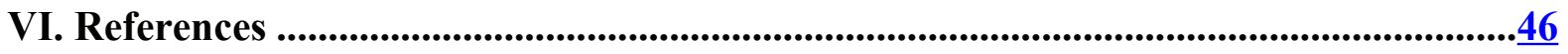

\section{Tables}

1. Correlates of Export Accelerations: Domestic Macroeconomic and Governance

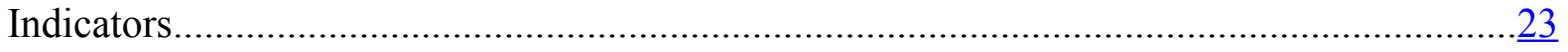

2. Correlates of Export Accelerations: Real Exchange Rate and Diversification....................24

3. Correlates of Export Accelerations: Trade Policy and Product Market Reforms................ 25

4. Correlates of Export Accelerations: Financial Liberalization ….................................... 27

5. Correlates of Export Accelerations: Globalization and GVC Participation .......................29

6. Country Weights in the Synthetic Control............................................................. 43

\section{Figures}

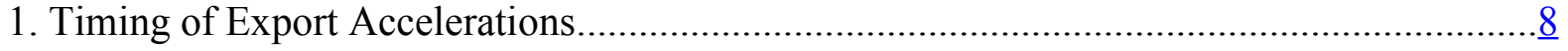

2. Distribution of Export Accelerations by Decade ........................................................ 8

3. Average Number of Export Accelerations per Country, by Region ................................10

4. Average Number of Export Accelerations per Country, by Income Level ........................10

5. Average Number of Export Accelerations per Country, by Main Source of Export Earnings .

6. Distribution of Export Accelerations in Latin America and the Caribbean.......................11 
7. Average Number of Export Accelerations per Country in LAC ….................................12

8. Around the Initiation Date of Goods Export Accelerations..........................................14

9. Around the Initiation Date of Services Export Accelerations .......................................15

10. Post-Acceleration Performance in Brazil and Peru ...................................................41

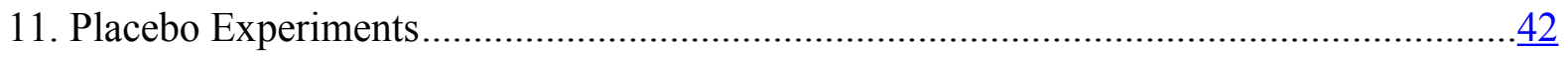

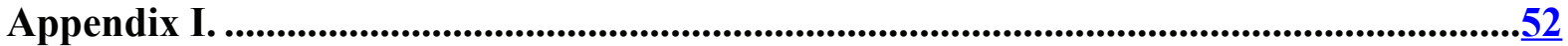




\section{INTRODUCTION}

1. The world economy has recently been marked by a global trade slowdown and sluggish output growth (IMF, 2016). Reinvigorating and sustaining strong export growth could be an engine of growth and productivity. But what factors lead to an export take off? This paper investigates the determinants of export accelerations by examining episodes of clear shifts in export growth. The rationale for this focus is similar to Hausmann et al. (2005) who examine the predictors of growth accelerations in GDP per capita, adopting Pritchett (2000)'s argument that output performance is not always stable, with countries alternately experiencing episodes of growth, stagnation, and decline of different durations. Beyond the abundant literature that exploits shifts in GDP per capita performance, ${ }^{2}$ other papers have focused on turning points in the savings rate (Rodrik, 2000; Ebeke, 2014), productivity growth (Cadot et al., 2015), changes in fiscal expenditure (Carrère and de Melo, 2012), and export growth (Freund and Pierola, 2012; Eichengreen and Gupta, 2013).

2. We contribute to the literature along several fronts. First, we explore a rich array of potential predictors of export accelerations instead of focusing on a single determinant. Second, we expand the analysis beyond manufacturing goods exports to include non-fuel primary commodities, which typically account for a large share of developing countries' export baskets. Third, we further extend our analysis to services exports, given the rising importance of trade in services (Sáez et al., 2015). Fourth, we allow for heterogeneities in the determinants of export accelerations by carrying out the empirical analysis for Latin America and the Caribbean (henceforth LAC) separately, and by distinguishing between goods and services.

\section{The paper finds that export accelerations are relatively frequent across the world, with a large bulk occurring in emerging market and developing economies.} Several preconditions including lower macroeconomic uncertainty, improved quality of institutions, a depreciated real exchange rate, agricultural reforms and global value chain (GVC) participation make the occurrence of export accelerations more likely. However, the paper also provides evidence of heterogeneity across regions. For instance, diversification matters for export transitions; but while the positive effect materializes through the intensive margin of trade for the world, diversification at the extensive margin seems to be key to achieving high and sustained export growth in LAC. Export takeoffs in services also tend to be associated with somewhat different triggers than those in goods. For example, services export accelerations in LAC are preceded by growth in FDI inflows and domestic financial liberalization through banking sector reforms whereas goods export surges respond to capital account openness. In the majority of cases, the effects of the correlates on the initiation of accelerations in LAC turn out to be at least twice the size of the estimates for the world

\footnotetext{
2 See for example Ben-David and Papell, 1998; Jones and Olken, 2005; Jerzmanowski, 2006; Jones and Olken, 2008; Guillaumont and Wagner, 2012; and Berg et al., 2012.
} 
sample, suggesting that the region would benefit more from the implementation of export growth promoting policies.

\section{The paper also assesses whether countries that experience export accelerations perform better in terms of higher GDP per capita and lower unemployment and income inequality. This contributes to the literature on the relationship between trade and growth, as well as trade and welfare (Bernard et al., 1995; Frankel and Romer, 1999; Winters, 2004). For this purpose, we resort to the synthetic control method developed by Abadie and Gardeazabal (2003) and extended by Abadie et al., (2010), and implement two illustrative case studies. We find that post-surge GDP per capita is higher in Peru, while the evidence is inconclusive for Brazil. In contrast, both countries experienced a lower unemployment rate and income inequality compared to their synthetic counterparts, highlighting the benefits of high and sustained export growth in terms of improving the income distribution and labor market conditions.}

5. The paper is organized as follows. Section 2 explains the methodology used to identify export accelerations and discusses some stylized facts, including findings from event analysis. Section 3 presents the empirical analysis of the determinants of export transitions. Section 4 assesses the post-surge performance of selected LAC countries using the synthetic control method. Section 5 concludes.

\section{IDENTIFICATION OF EXPORT ACCELERATION DATES}

\section{A. Methodology}

\section{Following Freund and Pierola (2012), we define an export acceleration as a} significant increase in export growth that is sustained for at least 7 years. ${ }^{3}$ Borrowing from Cadot et al. (2015), let $v_{i t}$ be the level of exports of country $i$ at time $t$, and $g_{i t}=$ $\ln \left(v_{i t}\right)-\ln \left(v_{i t-1}\right)$ the real growth rate of exports. ${ }^{4}$ The term takeoff refers to a seven-year period, with the surge date being its first year, and the baseline is the seven-year period immediately preceding it. Subsequently, we define $g_{i t}^{1}$ and $g_{i t}^{0}$ as the real average export growth during the takeoff and baseline periods respectively. Ultimately, the identification of the timing of export acceleration episodes relies on the simultaneous application of four criteria:
1. $g_{i t}^{1}>\bar{g}$
2. $g_{i t}^{1}>1.3 * g_{i t}^{0}$ and $g_{i t}^{1}>g_{i t}^{0}+0.03$

\footnotetext{
${ }^{3}$ We chose a seven-year horizon as in Hausmann et al. (2005) and Freund and Pierola (2012), but we also use five and nine year-periods for robustness purposes.

${ }^{4}$ Following Freund and Pierola (2012) exports are expressed in constant 2010 USD after deflating export values in current USD by the US CPI index.
} 
3. $\min \left(v_{i t}, v_{i t+1}, \ldots, v_{i t+6}\right)>\max \left(v_{i t-7}, v_{i t-6}, \ldots, v_{i t-1}\right)$

4. $g_{i t}^{1} \backslash\left\{\max \left(g_{i t}, g_{i t+1}, \ldots, g_{i t+6}\right)\right\}>g_{i t}^{0}$

- $\quad$ Criterion 1 ensures that real average export growth during takeoff is strong and above the world median value $\bar{g} .{ }^{5}$ Criterion 2 ensures that increases in export growth are substantial by imposing that the real average export growth during takeoff increases by one third from the baseline growth rate and exceeds it by at least 3 percentage points. ${ }^{6}$ To rule-out volatilitydriven surges, criterion 3 requires that the minimum level of exports observed during takeoff be higher than the maximum level of exports observed over the baseline period. Finally, criterion 4 avoids retaining accelerations triggered by a single year of very strong growth by imposing that the real average growth rate during takeoff, excluding the year of strongest growth, be greater than real average growth during baseline.

- $\quad$ To identify export accelerations, only countries with export spells of at least 14 years are considered, i.e. periods with missing observations are excluded. ${ }^{7}$ In the event of contiguous eligible years, we allow countries to have several instances of export accelerations as long as the dates are at least eight years apart. We investigate the timing of export accelerations for both goods and services exports. Mirror data on merchandise exports are taken from COMTRADE over 1976-2015. We focus on aggregate exports excluding fuels (SITC rev.2 section 3) and minerals (divisions 27, 28 and 68) to avoid identifying surges that are driven by global commodity price booms. Services export series are culled from the joint ITCUNCTAD-WTO dataset and span 1980-2013. Given data availability and the definition of the criteria, the earliest possible initiation date of a goods (services) export acceleration is 1983 (1987) and the latest 2009 (2007).

\section{B. Stylized Facts}

\section{The application of the filters on a sample of $\mathbf{1 8 7}$ countries yields $\mathbf{1 7 5}$ and $\mathbf{1 6 2}$} accelerations in goods and services exports, respectively. Figure 1 shows the timing of export accelerations, distinguishing between advanced economies, and emerging market and developing countries. The latter group comprised 86 percent of accelerations in goods exports and 73 percent in services. Accelerations were more frequent in the second half of the 1980 s, probably reflecting the transition from import substitution strategies to exportoriented growth. The first half of the 2000s also hosted a large number of accelerations, especially in services, possibly reflecting the rise of China and other emerging countries. More specifically, Figure 2 indicates that 78 out of the 175 goods accelerations and 88 out of

\footnotetext{
${ }^{5}$ Results remain unchanged when we use the world average export growth.

${ }^{6}$ The thresholds are taken from Freund and Pierola (2012), but we also conduct robustness checks by modifying the cutoff parameters.

${ }^{7}$ This choice may underrepresent countries with poor data coverage (which tend to be developing countries), countries created during the period under study, such as Post-Soviet States, or more recently, South Sudan.
} 
the 162 services accelerations occurred in the 2000s. Figure A1 in the Appendix further depicts the geographical distribution of export accelerations across the world.

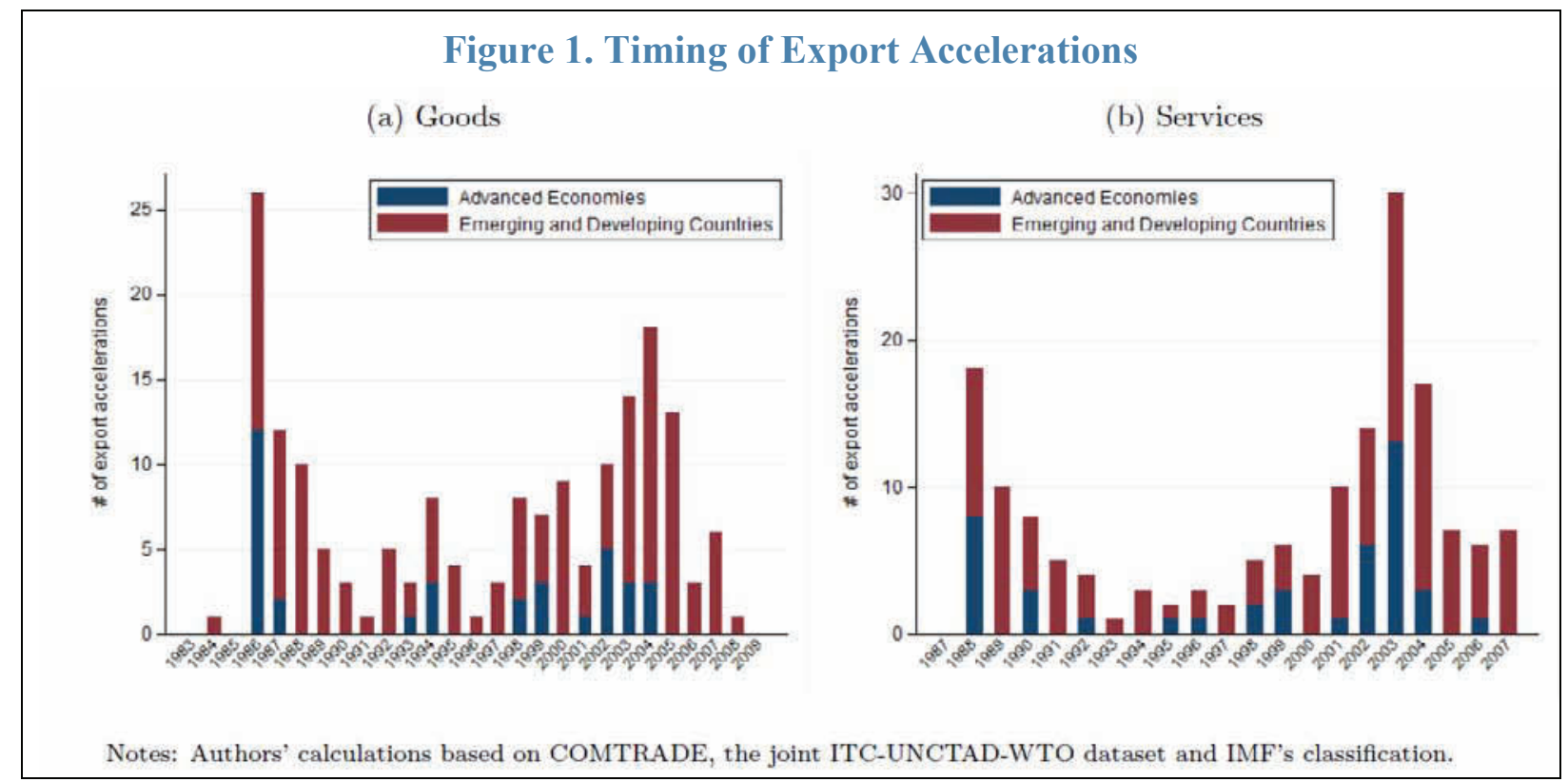

Figure 2. Distribution of Export Accelerations by Decade

(a) Goods

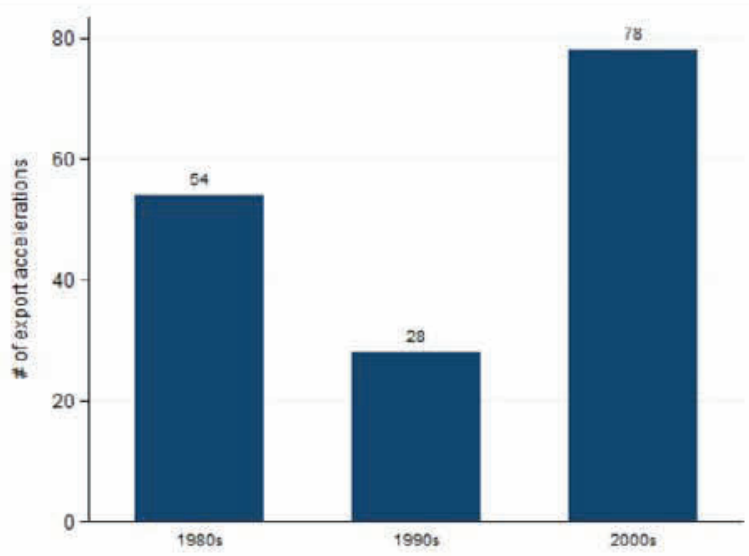

(b) Services

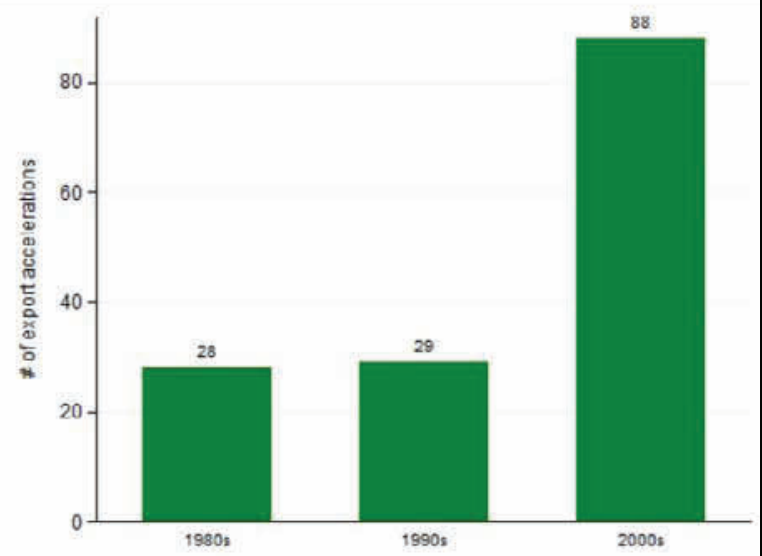

Notes: Authors' calculations based on COMTRADE and the joint ITC-UNCTAD-WTO dataset. Given the definition of the criteria applied in the identification of export surge dates, in (a) the 1980s start in 1983 and the 2000 s end in 2009 ; while in (b) the 1980 s start in 1987 and the 2000 s end in 2007.

\section{Next, we assess the regional distribution of export accelerations after} normalizing by the number of countries in each region (Figure 3). LAC appears as the best performer with an average of 1.19 goods accelerations per country, followed by 
Emerging Europe (1.18) and Middle East and North Africa (MENA) (1.14) ${ }^{8}$ Advanced economies rank first in terms of the average number of services export accelerations per country (1.19), followed by emerging Asia (1.03), MENA (0.82) and LAC (0.81). For both types of exports, the smallest figures were recorded by Sub-Saharan Africa. The rise in the average number of export episodes with the level of income suggests a positive association between the occurrence of accelerations and the level of development (Figure 4).

\section{How do emerging market and developing countries compare when grouped} according to their main source of export earnings? ${ }^{9}$ Figure 5 reveals that developing economies whose export revenues are sourced from manufactures and those with a diversified source of export earnings witnessed the highest average number of goods exports accelerations per country (1.44 and 1.09 respectively). In contrast, developing economies dependent on non-fuel primary commodities and services experienced the lowest average number of goods accelerations per country (0.72). A similar pattern holds for services accelerations, although in this case services-exporting economies performed better than oildependent countries.

\section{Focusing on LAC, Figure 6 reveals cross-country disparities within the region.} Most countries in Central and South America registered comparable performance in goods and services, as illustrated by the cases of Chile, Colombia, Peru, El Salvador and Nicaragua, among others. The number of services accelerations exceeded the number of goods accelerations in Brazil, whereas the inverse is true for Bolivia, Ecuador and Costa Rica. The latter stands out as the only country in LAC with three episodes of accelerations in goods exports. Interestingly, Caribbean countries that experienced accelerations registered a higher number of goods than services episodes. Haiti and Saint Vincent and the Grenadines are the only economies which performed better in services than in goods exports. LAC's performance in both goods and services is driven by LA6 countries (Figure 7). ${ }^{10}$ In contrast, the average number of goods and services export accelerations stands at only 0.67 and 0.17 , respectively, in the Caribbean region.

\footnotetext{
${ }^{8}$ Table A2 provides the list of countries included in each regional grouping.

${ }^{9}$ This is the October 2015 classification provided by the IMF's Statistics Department.

${ }^{10}$ LA6 comprises Brazil, Chile, Colombia, Mexico, Peru and Uruguay.
} 
Figure 3. Average Number of Export Accelerations per Country, by Region

(a) Goods

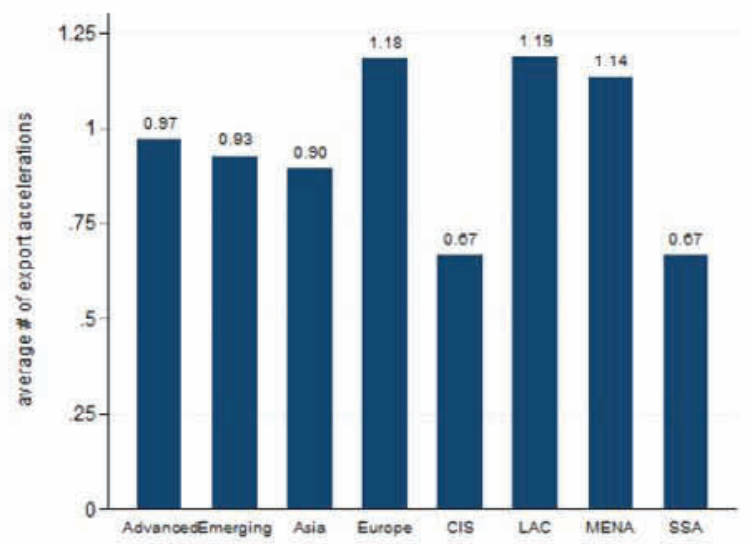

(b) Services

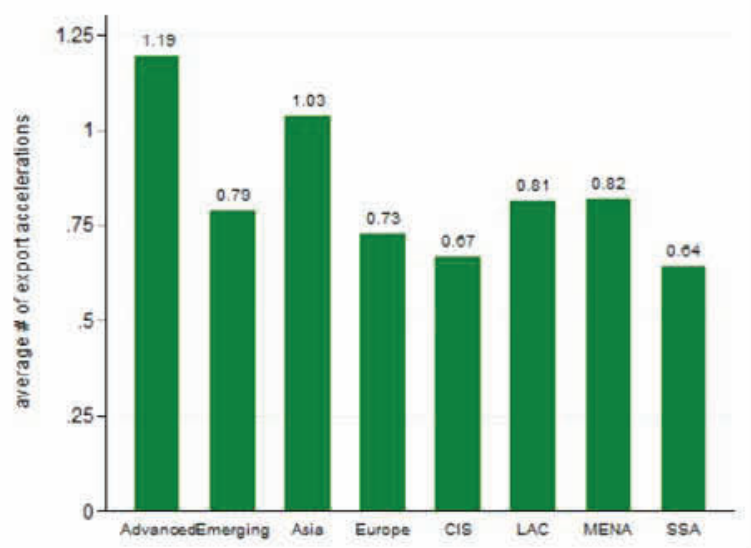

Notes: Authors' calculations based on COMTRADF, the joint ITC-UNCTAD-WTO dataset and IMF's classification. Fmerging market and developing countries are broken down into emerging Asia, emerging Europe, the Commonwealth of Independent States (CIS), Latin America and the Caribbean (LAC), Middle East and North Africa (MENA) and Sub-Saharan Africa (SSA). MENA includes Afghanistan and Pakistan. Table A1 details the countries included in each region.

Figure 4. Average Number of Export Accelerations per Country, by Income Level

(a) Goods

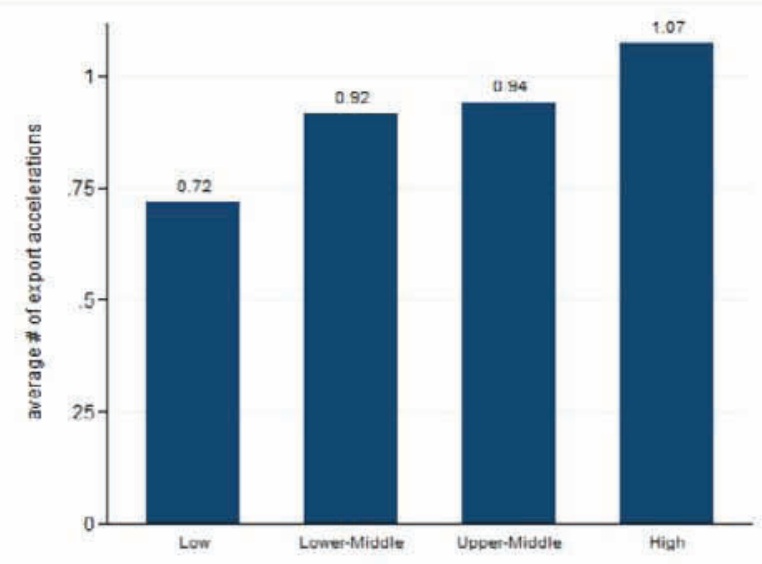

(b) Services

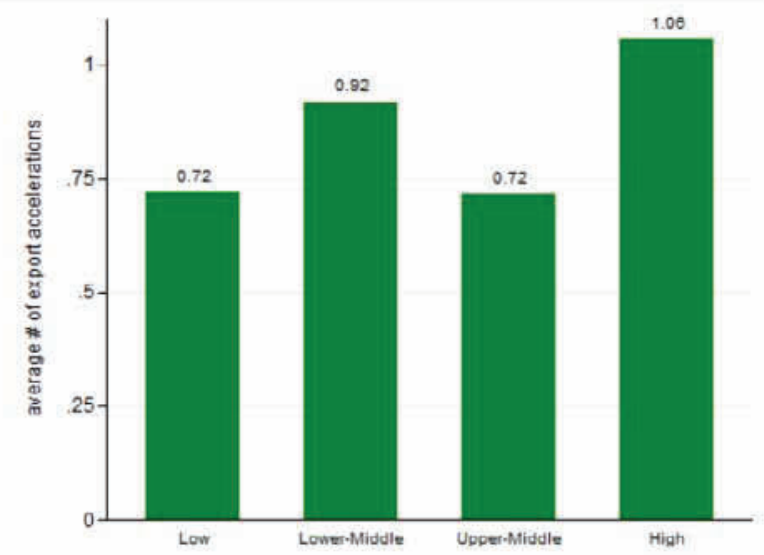

Notes: Authors' calculations based on COMTRADE, the joint ITC-UNCTAD-WTO dataset and the World Bank's 2013 income classification. Per capita GNI must be lewer than or equal to 1,045 USD to qualify as a low-income country, comprised leetween 1,046 and 4,125 USD for lower middle-income countries, comprised between 4,126 and 12,745 USD for upper middle-income countries; and higher than 12,745 USD for high-income countries. Table A1 details the countries included in each income group. 

Figure 5. Average Number of Export Accelerations per Country, by Main Source of Export Earnings

(a) Goods

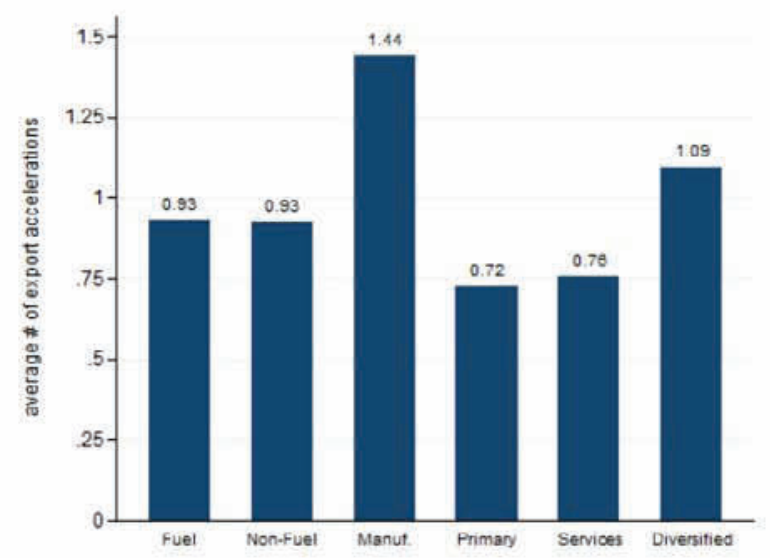

(b) Services

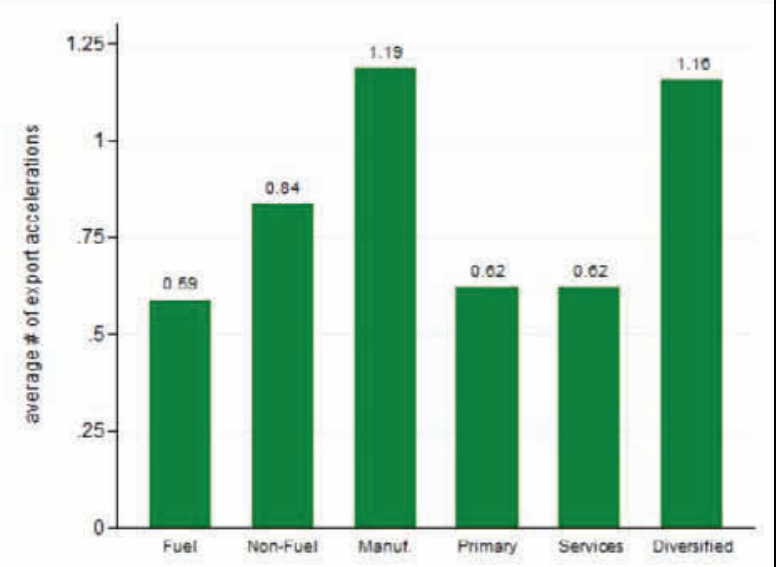

Notes: Authors' calculations based on COMTRADF, the joint ITC-UNCTAD-WTO dataset and IMF's classification. Non-fuel sources of export earnings include manufactures; non-fuel primary commodities; services, income and transfers, as well as a diversified source of export carnings. Table $A 1$ details the countries included in cach group.

Figure 6. Distribution of Export Accelerations in Latin America and the Caribbean

(a) Goods

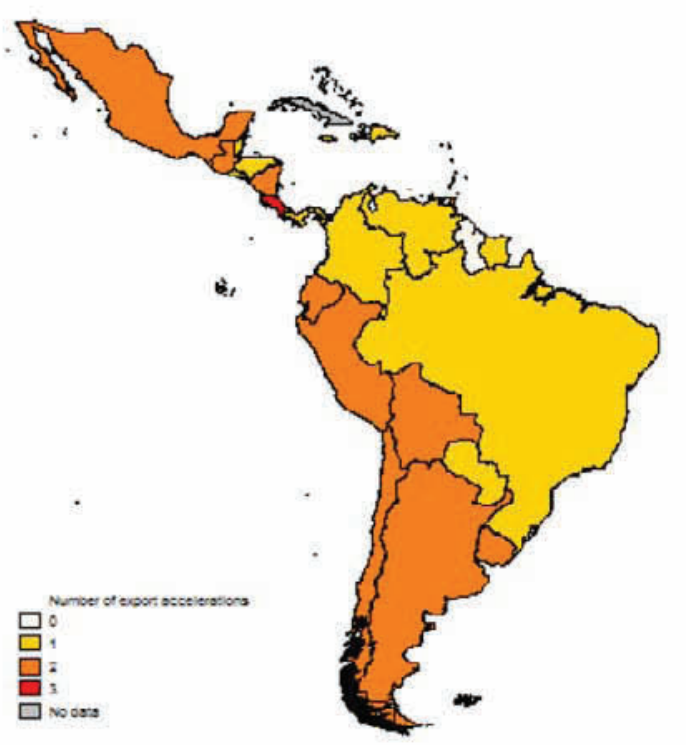

(b) Services

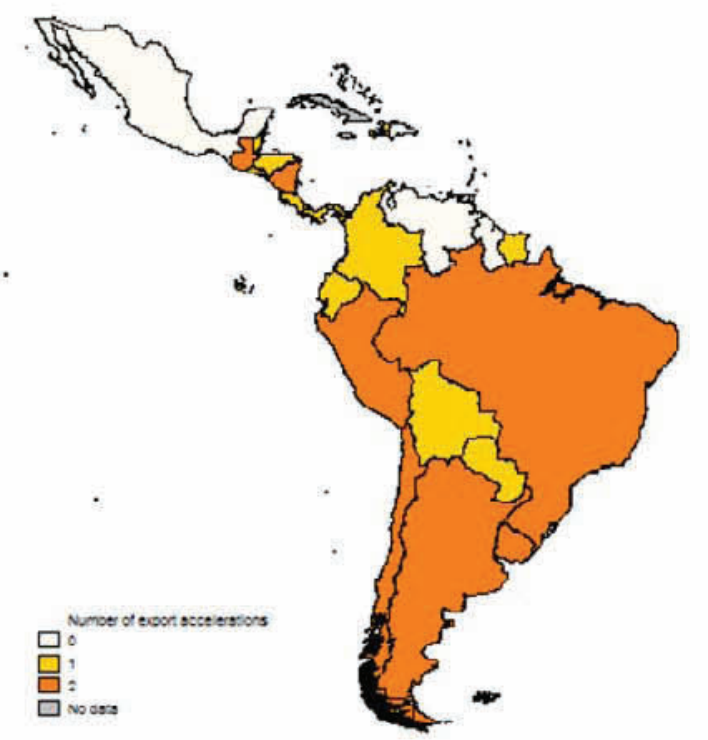

Notes: Authors' calculations based on COMTRADE and the joint ITC-UNCTAD-WTO dataset. Generated using STATA software. 
Figure 7. Average Number of Export Accelerations per Country in LAC

(a) Goods

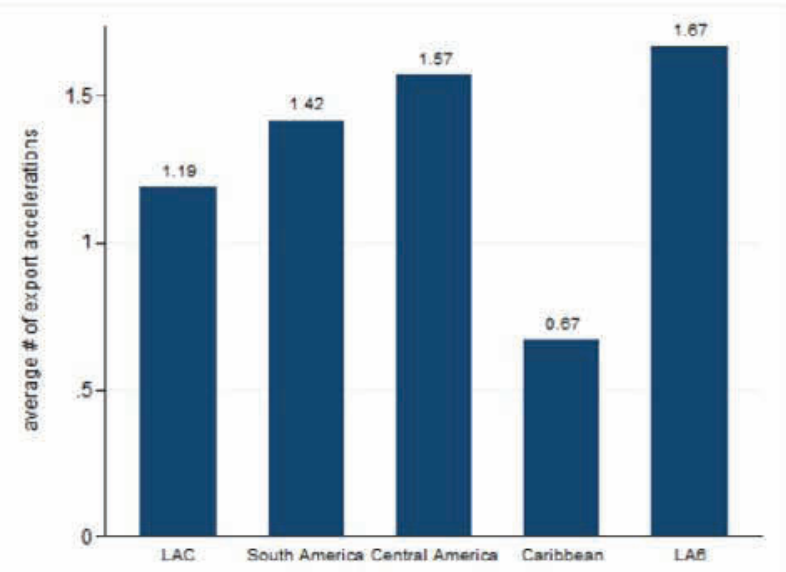

(b) Services

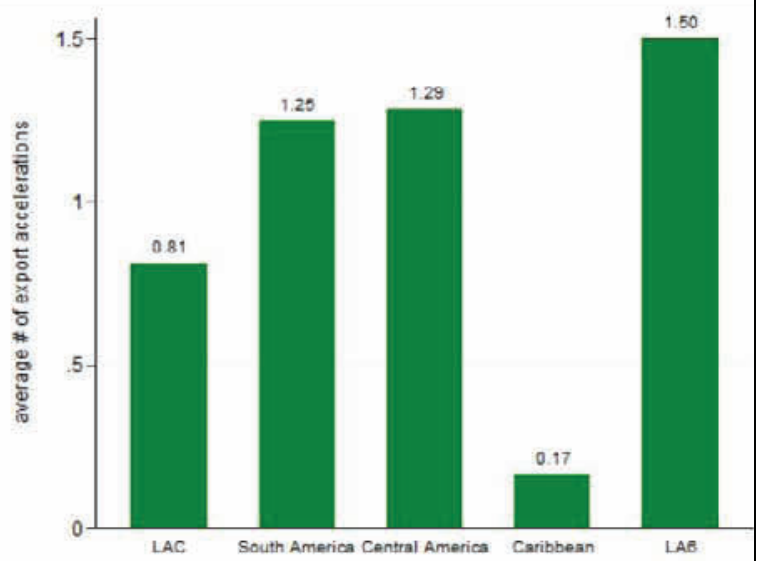

Notes: Authors' calculations based on COMTRADE, the joint ITC-UNCTAD-WTO dataset and IMF's classification. L 66 includes Brazil, Chile, Columbia, Mexico, Peru and Uruguay. Table A1 details the countries included in each sub-region.

\section{Event Studies}

11. In this section, we examine the time path of selected economic and social indicators of an average country that experienced an export acceleration. Similar to Wacziarg and Welch (2008) with trade liberalization dates, we use an event study methodology to depict the behavior of selected variables five years around the initiation date of the export surge. This exercise is carried out for both goods and services export acceleration dates with the aim of identifying potential predictors of export transitions, before turning to a more formal analysis of the determinants of export accelerations in Section 3.

\section{Figures 8 and 9 report the mean evolution of selected variables around the surge}

year. ${ }^{11}$ Analytical time is given on the $\mathrm{x}$-axis with $t=0$ being the initiation date. We split the sample of countries that experienced at least one export acceleration episode into LAC and non-LAC. The time path for an average country in LAC is illustrated by the solid red line (left-axis), whereas the dashed blue line pertains to an average country outside the region (right-axis). The dashed-dotted black line stands as a benchmark as it represents the time path for an average country in the sample considering all economies listed in Tables A1 and A2, i.e. including all countries with available data, irrespective of whether they experienced an acceleration or not. ${ }^{12}$ Axes are adjusted to reflect the same percentage change for the three series.

\footnotetext{
${ }^{11}$ A detailed description of each variable is available in Table A3.

${ }^{12}$ Furthermore, graphs are generated based on a balanced sample of episodes after restricting the sample to countries with continuously available data five years before and after the surge date. This ensures that depicted
} 
13. Graphs (a) and (b) display the mean evolution of the level and growth of exports around the initiation date. As expected, exports increase significantly at the surge time, and export growth accelerates as depicted by the sharp spike during takeoff. As in Cadot et al. (2015a, b), we observe a ratchet effect on real exports since levels seem to remain permanently higher after the initiation date, whereas mean reversion occurs in growth rates, for both goods and services. Before the surge date, the average LAC country typically records a real effective exchange rate (REER) depreciation of almost 20 percent and 12 percent for goods and services, respectively, (graph (c)); these figures are much larger than the 7 percent depreciation recorded for the benchmark. Similarly, the downward trend in the Theil index in graph (d) exhibits a larger slope for surge countries relative to the benchmark, suggesting that diversification is important for triggering export acceleration episodes. In the same vein, a reduction in tariffs occurs before the surge starts, especially for LAC, with rates falling by more than 3 percentage points while the reduction is less than 1 percentage point for the benchmark (graph (e)). A rise in GVC participation characterizes the baseline period (graph (f)).

\section{Graphs (g) - (i) show the average behavior of real GDP per capita, unemployment and income inequality five years before and after a surge starts. The} post-acceleration trajectories of these three variables are formally assessed in Section 4 using the synthetic control methodology, but Figures 8 and 9 offer a first look at the data. Although real GDP per capita of LAC countries rises at a similar rate as the benchmark after an export surge, non-LAC economies grow considerably faster, especially after accelerations in goods exports. Unemployment recorded a remarkable fall in both LAC and non-LAC surge countries, with a 1.5 percentage point decrease over the post-surge phase, while the benchmark rate only decreased by 0.2 percentage point over the same period. Furthermore, income inequality in surge-countries decreased by an approximate 6.7 percent during the five-year period that followed the goods export acceleration date, while the Gini index remained mostly unchanged for the benchmark. In the case of services, LAC countries enjoyed a 7.6 percent reduction in income inequality during the post-acceleration phase, while the index slightly increased for the benchmark.

movements reflect within-country changes only, abstracting from variations that may be induced by the addition or subtraction of particular observations (Freund and Pierola, 2012). The list of countries included in each graph is available upon request. We also produce graphs based on the median evolution of variables to account for possible effects of outliers. Results are available upon request. 


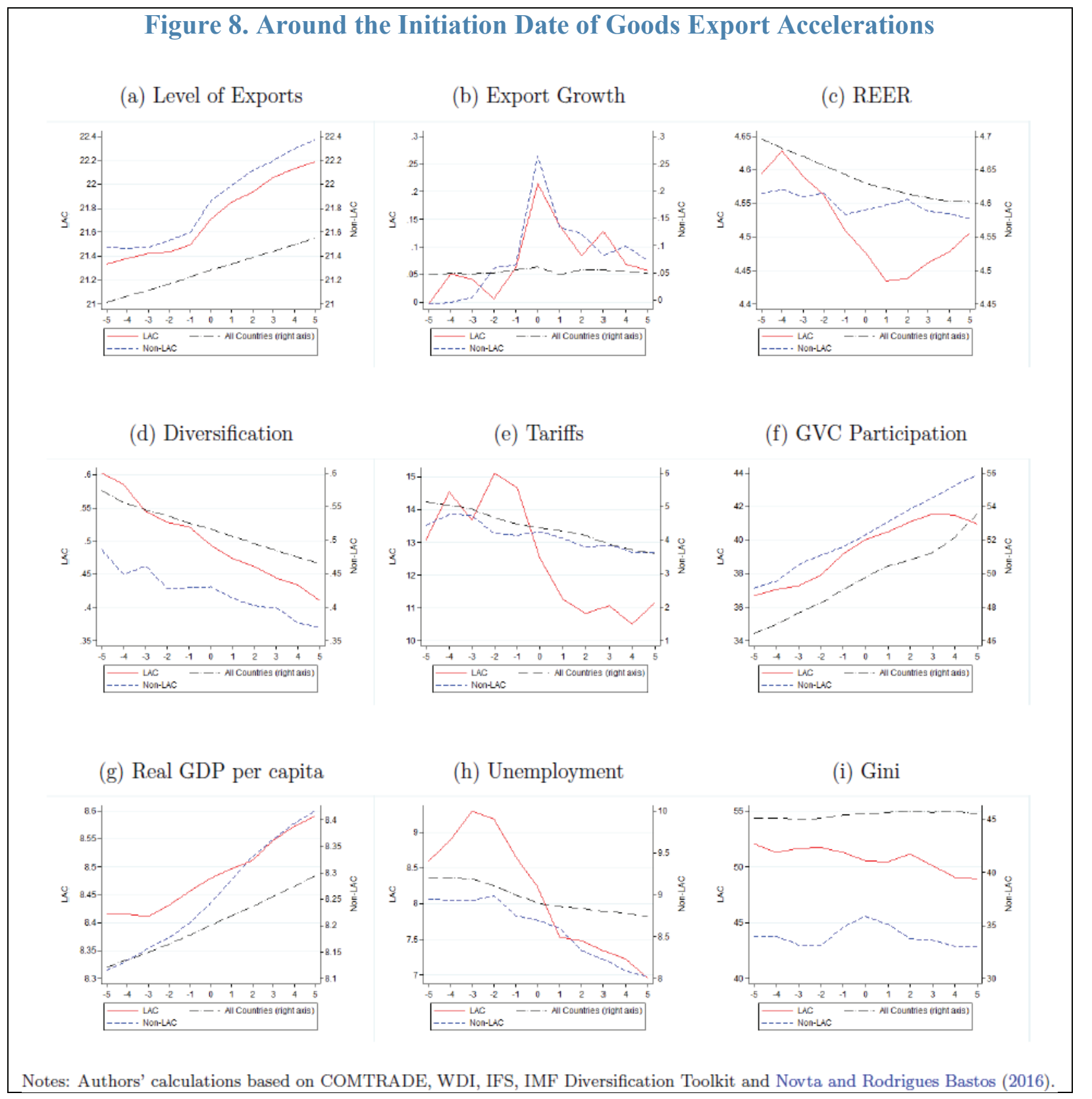

CInternational Monetary Fund. Not for Redistribution 
Figure 9. Around the Initiation Date of Services Export Accelerations

(a) Level of Exports

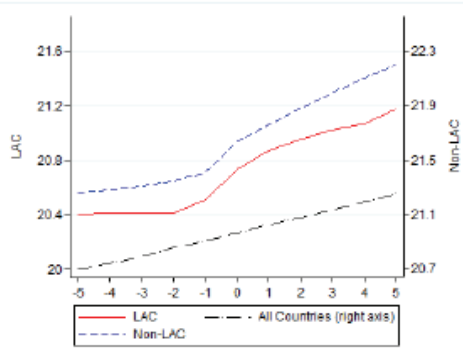

(d) Diversification

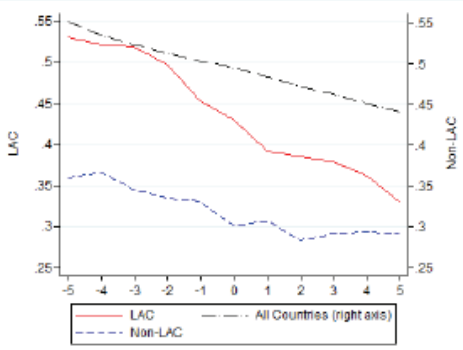

(g) Real GDP per capita

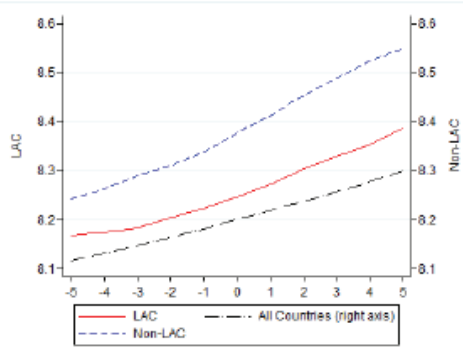

(b) Export Growth

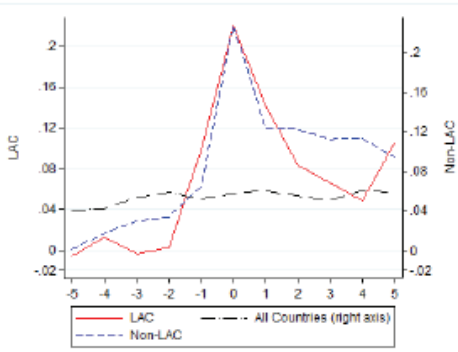

(e) Tariffs

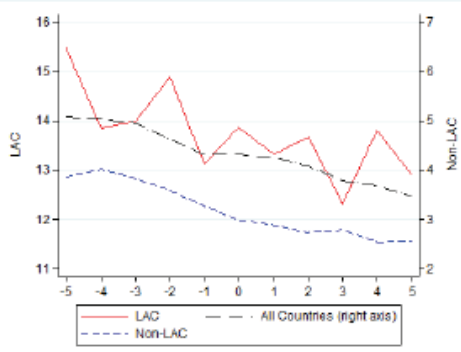

(h) Unemployment

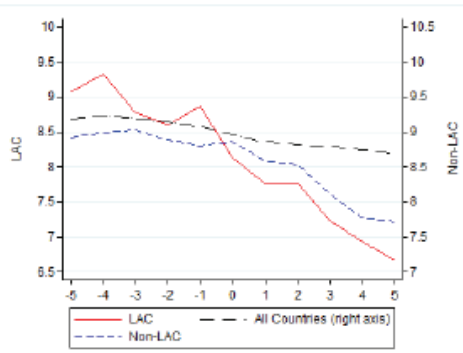

(c) REER

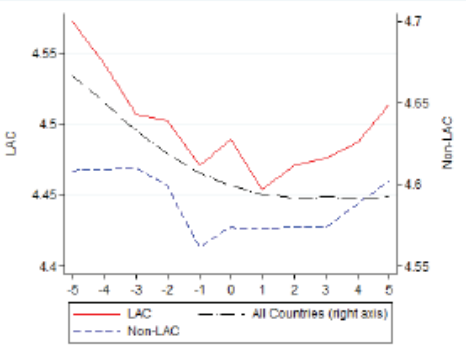

(f) GVC Participation

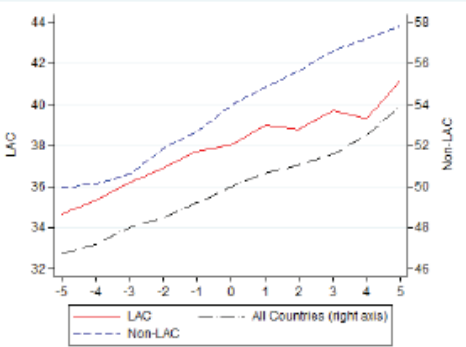

(i) Gini

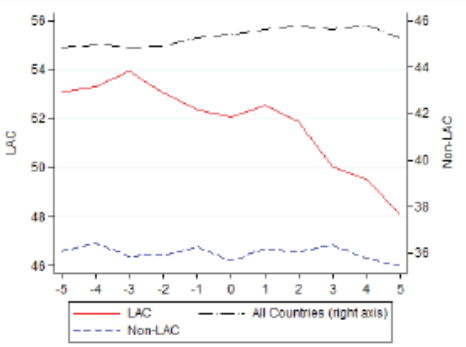

Notes: Authors' calculations based on COMTRADE, the joint ITC-UNCTAD-WTO dataset, WDI, IFS, IMF Diversification Toolkit and Novta and Rodrigues Bastos (2016).

\section{CORRELATES OF EXPORT ACCELERATIONS}

\section{A. Econometric Model}

15. In this section, we formally investigate the determinants of export transitions using regression analysis. Specifically, we estimate the following probit model of the timing of export accelerations:

$$
\begin{aligned}
& \operatorname{Pr}\left(E_{i t}=1\right)=\phi\left[\delta_{0} \ln \left(\text { GDP }_{\text {cap }_{i t-2}}\right)+\delta_{1} \ln \left(\text { GDPcap }_{i t-2}^{2}\right)+\delta_{2} \ln \left(\text { Population }_{i t-2}\right)\right. \\
& \left.+\delta_{3} \text { Market Access }_{i t-2}+\delta_{4} X_{i t-2}+\sum \lambda_{t} D_{t}\right]
\end{aligned}
$$


where $\phi$ is the cumulative normal distribution. The dependent variable $E A_{i t}$ is a dummy that equals 1 over the three-year window centered on the initiation year of the export acceleration (i.e. for $t-1, t$ and $t+1$ ). As in Hausmann, Pritchett and Rodrik (2005), Carrère and de Melo (2012) and Ebeke (2014), we impose a three-year window to reduce the likelihood of narrowly missing the timing of an acceleration through quirks in the data or in our method. The sample is not restricted to countries that have experienced export accelerations ${ }^{13}$, but we adjust it as follows: (i) we drop the first and last seven years of data as export acceleration episodes could not have been calculated for those years given the criteria we applied to identify them; (ii) since we are interested in uncovering the variables that contribute to triggering export takeoffs, we drop all data pertaining to years $t+2, \ldots, t+7$ of an episode.

\section{A parsimonious baseline specification controls for country size, the level of development, by allowing for non-linear effects of income per capita, and market} access. Fernandes et al. (2016) show that country size and stage of development matter as larger countries and developed economies export more because they host large firms that account for a significant share of exports. Export survival also tends to be lower at an early stage of development, suggesting a positive relationship between income and export accelerations. In addition, the baseline model also accounts for country membership in economic integration agreements, computed as the weighted sum of all economic agreements a country participates in, with the weights corresponding to the partner's market size (Cadot et al., 2014). The literature provides mixed evidence on the relationship between economic integration agreements and trade flows. For instance, Baier and Bergstrand (2007) find a positive impact of FTAs on members' international trade and Hannan (2016) demonstrates that trade agreements boost exports; however, other studies document limited or even negative effects on trade flows (Bergstrand, 1985; Frankel et al., 1995, 1997). Year dummies $D_{t}$ are included to capture time-varying unobserved heterogeneity common to all countries, such as international commodity price shocks.

We test a large number of potential predictors after organizing them in five categories. Investigated determinants of the timing of export accelerations are captured by $X_{i t-2}$. They are entered one at a time in the baseline model to avoid multicolinearity. The five categories, elaborated below, are domestic macroeconomic and governance indicators, real exchange rate and diversification, trade policy and product market reforms, financial liberalization, and globalization and GVC participation.

\section{Domestic Macroeconomic and Governance Indicators}

16. Investment growth, a sound macroeconomic environment, quality infrastructure and institutions as well as human capital are positively correlated with the probability of observing an export acceleration. ${ }^{14}$ Human capital is proxied by the secondary school

\footnotetext{
${ }^{13}$ Tables A1 and A2 give the list of countries included in the analysis.

${ }^{14}$ Human capital is included as a standard control variable in assessing the effect of the potential determinants classified in the remaining tables.
} 
enrollment rate taken from WDI. Lennon (2009) finds that secondary school enrollment positively influences services trade. More generally, the availability of skilled labor appears critical to services exports relying on IT (Sáez et al., 2015). Investment in hard and soft infrastructure including equipment purchases, land improvements and construction of roads should raise the supply capacity of a country. We complement this WDI indicator with an index of infrastructure quality taken from Carrère et al. (2009). ${ }^{15}$ The importance of traderelated infrastructure in supporting exports is highlighted by Freund and Weinhold (2002) who find that the Internet spurs growth in services trade. In the same vein, Lennon et al. (2009) show that the quality and quantity of transportation and telecommunications infrastructure matter for trade. We consider the three-year change in both variables to allow time for the effects to be felt. Similarly, a sound macroeconomic environment should raise the likelihood of export transitions. We use the REER volatility as a proxy for uncertainty. It is calculated as the standard deviation of the annual REER over the past five years using data from the IMF's IFS. Two indicators are used for institutional quality, namely Polity 2 from Marshall and Jaggers (2002) which measures the degree of democracy, and the ICRG indicator of quality of government. The latter is computed as the average of the variables "Corruption", "Law and Order" and "Bureaucracy Quality".

\section{Real Exchange Rate and Diversification}

\section{We also examine whether the exchange rate and export diversification help} predict export takeoffs. A large body of literature has investigated the relationship between the exchange rate and international trade. Freund and Pierola (2012) find that exchange rate depreciation is positively associated with subsequent manufactures export growth in developing countries. They show that depreciation stimulates entry into new export products and markets, which account on average for 40 percent of export growth. Similarly, Eichengreen and Gupta (2013) confirm the positive and significant effect of real exchange rate depreciation on export growth, with a larger effect for services. We use the IMF REER index to test this hypothesis. We also draw data from the Penn World Tables 8.0 (Feenstra et al., 2015). Specifically, we use the real exchange rate at PPP to compute real exchange rate misalignment adjusting for the Balassa-Samuelson effect as in Rodrik (2008). Furthermore, countries with a diversified export portfolio may be more likely to experience episodes of high and sustained export growth. We exploit the IMF Diversification Toolkit where the aggregate Theil index further maps into the intensive and extensive margins of export diversification. ${ }^{16}$

\footnotetext{
${ }^{15}$ The index is computed as the mean of four variables: i) the number of main telephone lines per 1000 workers, ii) the length of the road network, iii) the length of the railway network and iv) the share of paved roads in total roads.

${ }^{16}$ Similar to Cadot et al., 2011, we refer to the intensive and extensive margins of export diversification as the "within" and "between" components of the aggregate Theil index of export concentration.
} 


\section{Trade Policy and Product Market Reforms}

18. Next, we assess whether trade openness contributes to raising the probability of experiencing export accelerations. For this purpose, we use the three-year change in the ratio of goods and services trade to GDP, and data on average applied tariff rates taken from WDI. ${ }^{17}$ In particular, we examine if lowering tariff rates on manufactures and primary products both contribute to initiating export accelerations.

19. Export growth may also depend on product market competition and the quality of telecom and electricity services. We exploit Prati et al. (2013)'s database to investigate the role of structural reforms that stimulate product market competition. We use the agricultural reform index which measures the extent of public intervention in the market of the country's main agricultural export commodity. The presence of export marketing boards and the incidence of administered prices are captured by the measure. We also investigate whether the degree of liberalization in the telecommunication and electricity markets captured by the extent of competition in the provision of these services, privatization and the existence of an independent regulator - matters for export transitions. Services liberalization is found to benefit firms in deregulated sectors through a direct competition effect that induces innovation and the adoption of new technologies (Lanau and Topalova, 2016), possibly triggering export accelerations. Also, downstream firms using the output of deregulated sectors enjoy greater availability and higher quality of inputs. For instance, Arnold et al. (2008) find that reduced barriers to competition in telecommunication services in SSA boost manufacturing productivity. In the same vein, Arnold et al. (2011) and Arnold et al. (2016) show that liberalization in services industries positively impacts the productivity of manufacturing firms in the Czech Republic and India respectively.

\section{Financial Liberalization}

\section{Financial openness - the deregulation of domestic financial markets and the} liberalization of the capital account (Rancière et al., 2008) - may also play a role in igniting export acceleration episodes. Financial liberalization reduces the cost of capital through improved risk sharing and increased availability of foreign capital (Bekaert and Harvey, 2000; Henry, 2000; Bekaert et al., 2005). For example, Laeven (2003) finds that the liberalization of the banking sector reduces firms' financing constraints. Financial openness bolsters trade by alleviating credit market imperfections, consistent with the micro literature

\footnotetext{
${ }^{17}$ An alternative would be to use the Sachs and Warner (1995) trade liberalization dates, updated by Wacziarg and Welch (2008). A trade liberalization date is defined as the first year when none of the following characteristics are in place: i) average tariff rates of 40 percent or more; ii) non-tariff barriers covering 40 percent or more of trade; iii) a black market exchange rate at least 20 percent lower than the official exchange rate; iv) a state monopoly on major exports; and v) a socialist economic system. A liberalization date hence reflects the transition towards openness via broad economic reforms (Hausmann et al., 2005). Freund and Pierola (2012) find a positive relationship between trade liberalization and manufactures export growth. However, we do not use the liberalization dates as we are interested in examining the distinct effects of openness, tariffs and exchange rates on export accelerations.
} 
that documents the adverse effects of financing constraints on export participation (see for example Minetti and Zhu, 2011 on Italy; Muûls, 2015 on Belgium; Manova et al., 2015 on China; and Kiendrebeogo and Minea, 2016 on Egyptian manufacturing firms). For instance, Manova (2008) shows that equity market liberalizations stimulate aggregate exports, especially for sectors that are more dependent on external finance.

\section{On the other hand, financial liberalization may deter export accelerations.}

Financial liberalization may encourage excessive risk-taking, leading to more volatile capital flows that are prone to sudden reversals (IMF, 2012). Massive capital inflows following capital account liberalizations may lead to exchange rate appreciation and undermine the competitiveness of the tradable sector (Ostry et al., 2010); they may also fuel credit booms and asset price bubbles which can amplify financial fragility and crisis risk (Dell'Ariccia et al., 2012; Mendoza and Terrones, 2012; Schularick and Taylor, 2012). Kaminsky and Reinhart (1999) find that financial liberalization often precedes banking crises, which have been shown to jeopardize firms' export activity through reduced access to credit, especially trade finance (Iacovone and Zavacka, 2009; Amiti and Weinstein, 2011; Chor and Manova, 2012; Kiendrebeogo, 2013).

\section{We consider two measures of financial sector reforms, namely the index of domestic financial liberalization and capital account openness, both from Prati et al.} (2013). Domestic financial liberalization covers reforms pertaining to the banking sector and the securities market. The former measures the reduction or removal of i) interest rate controls such as floors or ceilings; ii) credit controls; iii) competition restrictions such as entry barriers in the banking sector; iv) the degree of state ownership; and v) a measure of the quality of banking supervision and regulation. Financial reforms relating to the securities market capture policies designed to promote the development of bond and equity markets, and access of the domestic stock market to foreigners. The capital account openness index measures the extent to which residents and non-residents can freely move capital into and out of the country. We use the aggregate index and its two sub-components relating to residents and non-residents.

\section{Globalization and GVC Participation}

23. The last set of variables pertains to globalization and GVC participation. We use the three-year change in foreign direct investment (FDI) inflows (percent of GDP) from UNCTAD. FDI may contribute to changing a country's export basket composition depending on the sector to which it is directed. It is usually expected to support a transition toward higher value-added activities through technological and knowledge spillovers, hence affecting export performance (Fugazza, 2004). Van der Marel (2012) finds a positive association between inward FDI and productivity in services, while Fernandez and Paunov (2012) show that FDI inflows in services boost manufacturing firms' productivity in Chile, therefore suggesting a possible export acceleration-triggering effect of FDI. We also use the KOF index of globalization introduced by Dreher (2006) and updated in Dreher et al. (2008). 
It covers the economic, social and political dimensions of globalization as captured by the flow of trade, capital, information and people. ${ }^{18}$

\section{Next, we examine whether participation in cross-border production chains has a} bearing on export accelerations. We use data from Novta and Rodrigues Bastos (2016) who rely on Koopman et al. (2014)'s decomposition of gross exports to distinguish between foreign and domestic value-added exports (FVA and DVA respectively). ${ }^{19} \mathrm{FVA}$ is used as a proxy for downstream involvement of countries in GVCs since it represents the share of gross exports which consists of inputs that have been produced in other countries. In contrast, DVA pertains to the share of gross exports that is created in-country. Subsequently, we look into the export-triggering potential of "indirect value-added exports" or DVX, which is the portion of DVA that enters as an intermediate input in the value-added exported by other countries (Koopman et al., 2010; UNCTAD, 2015; IMF, 2015). Of particular interest is the component of DVX that is re-exported to third countries, the so-called Term 3 in Koopman et al. (2014)'s nine-term decomposition of gross exports. We use this variable as a proxy for a country's participation in longer value chains as in IMF (2015). Our overall measure of GVC participation consists of the sum of FVA and DVX, hence reflecting both downstream and upstream involvement in multi-stage trade process.

25. Table A3 provides the description and source of variables. All explanatory variables are lagged by two years to mitigate reverse causality issues, but our analysis may not be entirely immune to endogeneity stemming from simultaneous bias, especially considering variables such as FDI inflows that may be forward-looking. The results should be interpreted accordingly and with caution.

\section{B. Baseline Results}

\section{Tables 1 to 5 display the main probit results for the world sample and for LAC} separately. While Panel A reports the marginal coefficients from the estimation of the probit model of goods export accelerations, Panel B shows the results for export surges in services. Additional statistics are provided at the bottom of each panel. They include the number of export acceleration episodes included in each regression, as well as the pseudo $R^{2}$ and McFadden's pseudo $R^{2}$ which measure the model's fit. The predictive ability of the probit model is gauged with the percentage of cases correctly classified, i.e. the proportion of export acceleration observations that are correctly predicted. ${ }^{20}$

\footnotetext{
18 Table A3provides more detail about the included sub-components.

19 The authors use the Eora Multi Region Input Output Table (Lenzen, Kanemoto, Moran and Geschke, 2012 , 2013).

${ }^{20}$ The percentage of cases correctly classified is identified by using the share of observations for which $E A_{i t}=1$ as the cutoff value for determining whether the predicted outcome is positive.
} 


\section{Coefficients on the baseline regressors are broadly significant and bear the} expected sign. Population consistently enters with a statistically significant and positive sign, suggesting that large economies have a higher probability of witnessing export takeoffs. Countries belonging to free trade areas and customs unions are also more likely to enjoy instances of high and sustained export growth. This result is fairly consistent for goods exports but seldom holds in the case of services due to loss of significance. ${ }^{21}$ Moreover, probit estimates for the timing of goods export accelerations reveal a hump-shaped relationship between the occurrence of surges and income per capita, while the association is convex for LAC. Again, results for services exports are rather mixed, although some evidence seems to suggest a U-shaped pattern.

\section{Reduced macroeconomic uncertainty and the quality of human capital,} infrastructure, and government positively correlate with the probability of observing a goods export acceleration in the world (Table 1 Panel A). Results broadly hold for LAC although the coefficient on the change in the quality of infrastructure is not statistically significant anymore. ${ }^{22}$ In contrast, transition towards democracy particularly matters for launching sustained export growth in the region as a one-unit increase in the polity 2 score raises the likelihood of observing an export surge by 2.4 percentage points. Panel B indicates that services export accelerations are also more likely to occur in a context of low macroeconomic uncertainty. The twofold increase in the estimate for government quality suggests that services are more sensitive to the quality of institutions than goods. This result is in line with Amin and Mattoo (2006) who find that countries with better institutions have larger and more dynamic services sectors, and Nunn (2007) who argues that services require strong institutions. Similarly, while investment growth is positively associated with services export surges in both samples, its effect is three times larger for LAC and stands at 11.7 percentage points. Although its positive effect fails to materialize in LAC, human capital seems to matter for services export accelerations across the world, consistent with Lennon (2009); and Jensen (2008) who shows that services tend to be more skill-intensive than goods.

29. Export competitiveness is associated with export surges (Table 2). A 10 percent depreciation in the REER raises the probability of observing a goods export acceleration by 1.4 percentage points in the overall sample, against 3.2 percentage points in the LAC region. Estimates obtained with the RER convey the same qualitative message that currency depreciation contributes to launching export accelerations in Latin America and the world,

${ }^{21} \mathrm{We}$ also note some cases of sign reversals, but exclusively for the estimations carried out over the sample of LAC countries. This result seems to echo the literature on the negative effect of economic integration agreements on trade flows (Frankel et al., 1997); but we also suspect such cases to be in part related to the limited sample size and the shrinking number of export accelerations included in LAC regressions.

${ }^{22}$ Surprisingly, the infrastructure index is negatively associated with the timing of services export accelerations in LAC. 
the effect being stronger for the former. This result confirms the event analysis undertaken in Section 2.3 and is in line with Freund and Pierola (2012) who uncover a more pronounced role of the exchange rate in stimulating export growth in developing countries. We also find evidence of the acceleration-hindering effect of RER misalignment for both samples. ${ }^{23}$ Panel $\mathrm{B}$ echoes similar findings for services export surges, with, again, magnified effects for LAC since estimates are twice the size of those found for the world sample.

\section{Table 2 also sheds light on the role of export diversification in initiating export}

takeoffs. Panel A shows that, on average, diversification at the intensive margin matters: a one-unit fall in the "within" Theil index of export concentration translates into a 3.1 percentage points increase in the likelihood of observing a goods export acceleration. The intensive margin clearly drives the result at the aggregate level, as the coefficient on the "between" Theil bears the expected sign but is not statistically significant. In contrast, for the LAC sample, diversification seems to promote export surges through the extensive margin of trade: a one-unit reduction in the "between" Theil index leads to a 14 percentage point rise in the probability of experiencing an acceleration. In other words, if LAC countries raise the range of products they export, they would increase their probability of experiencing a sustained goods export growth. The analysis in terms of Theil indices may not be directly relevant for services accelerations as the IMF Diversification Toolkit provides data for merchandise exports only. Nonetheless, the statistically significant results reported in Panel B suggest spillover effects from goods to services as product diversification seems to promote the initiation of services export acceleration episodes.

\section{Results for trade openness and product market reforms are summarized in}

Table 3. Growth in the trade-to-GDP ratio positively correlates with export accelerations in LAC. Specifically, a 1 percent rise in trade openness growth translates in a 25.2 and 27.3 percentage point increase in the probability of experiencing an acceleration in goods and services exports respectively.

32. High tariffs appear to deter exports. Despite the drastic reduction in sample size, estimates provide some insights in the importance of lowering barriers to trade, consistent with the event analysis carried out in Section B. Panel A shows that average applied tariff rates negatively correlate with the probability of experiencing a goods export acceleration. Reducing tariffs on manufactures seems to matter most. Panel B indicates that tariffs on primary products hurt services export performance in LAC, with a coefficient standing at 3 percentage points. This might reflect complementarities between services exports and goods imports in the region. For instance, high tariffs on imported energy may be an impediment for initiating sustained growth in the export of transportation or communication (postal and courier) services for which fuel may be a major intermediate input.

\footnotetext{
${ }^{23}$ The estimate for LAC is significant at the unconventional 11 percent level.
} 


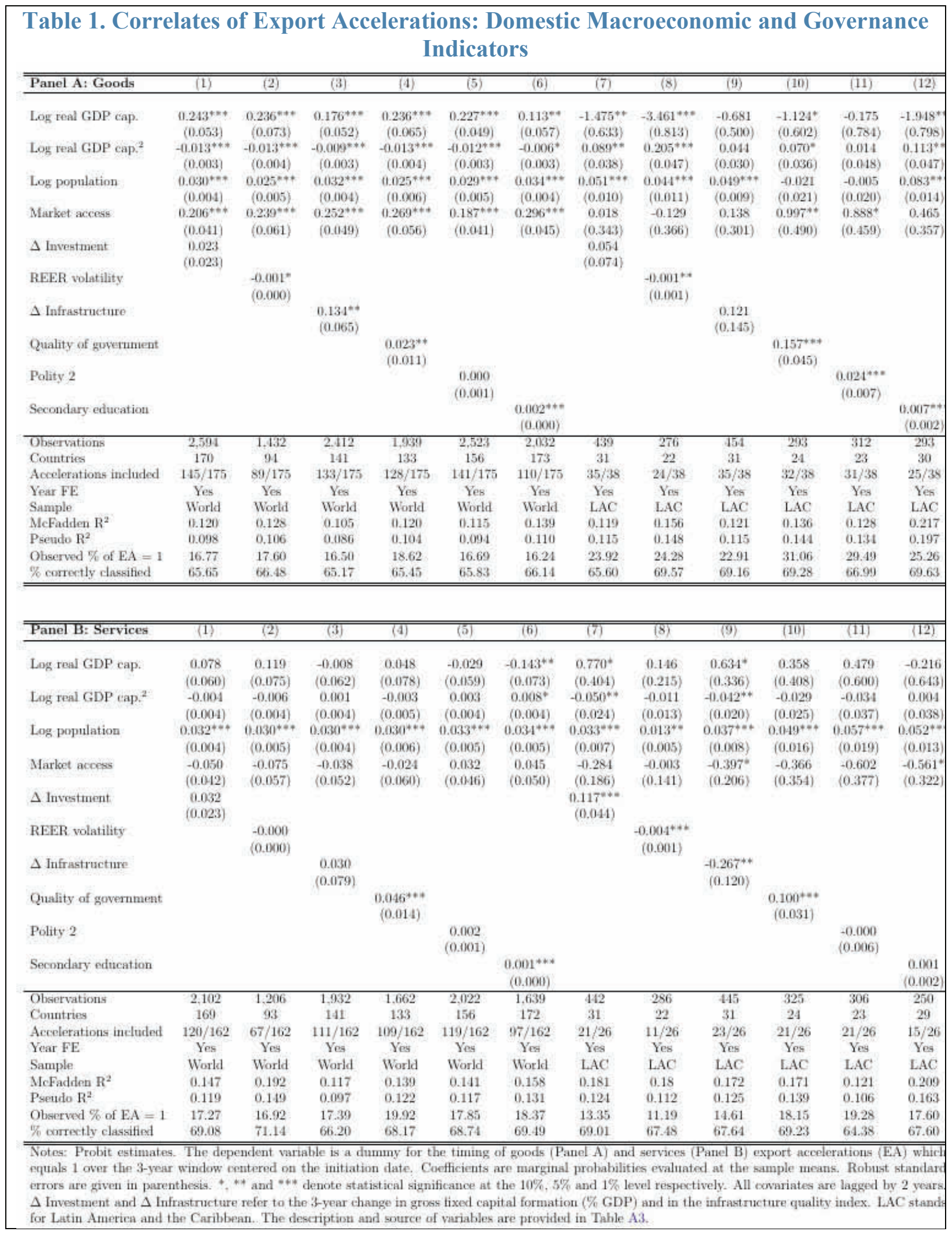


Table 2. Correlates of Export Accelerations: Real Exchange Rate and Diversification

\begin{tabular}{|c|c|c|c|c|c|c|c|c|c|c|c|c|}
\hline Panel A: Goods & (1) & $(2)$ & (3) & $(4)$ & $(5)$ & $(6)$ & $(7)$ & $(8)$ & $(9)$ & $(10)$ & $(11)$ & $(12)$ \\
\hline Log real GDP cap. & $\begin{array}{c}0.117 \\
(0.098)\end{array}$ & $\begin{array}{c}0.076 \\
(0.065)\end{array}$ & $\begin{array}{c}0.078 \\
(0.066)\end{array}$ & $\begin{array}{c}0.103 \\
(0.067)\end{array}$ & $\begin{array}{c}0.104 \\
(0.065)\end{array}$ & $\begin{array}{l}0.112^{*} \\
(0.067)\end{array}$ & $\begin{array}{c}-3.987^{* * *} \\
(0.993)\end{array}$ & $\begin{array}{c}-2.662^{* * *} \\
(1.053)\end{array}$ & $\begin{array}{c}-2.647^{* *} \\
(1.053)\end{array}$ & $\begin{array}{c}-1.925^{* *} \\
(0.792)\end{array}$ & $\begin{array}{c}-2.378^{* * *} \\
(0.826)\end{array}$ & $\begin{array}{r}-1.977^{* * *} \\
(0.817)\end{array}$ \\
\hline Log real GDP cap. ${ }^{2}$ & $\begin{array}{l}-0.007 \\
(0.006)\end{array}$ & $\begin{array}{c}-0.003 \\
(0.004)\end{array}$ & $\begin{array}{c}-0.004 \\
(0.004)\end{array}$ & $\begin{array}{l}-0.006 \\
(0.004)\end{array}$ & $\begin{array}{l}-0.005 \\
(0.004)\end{array}$ & $\begin{array}{l}-0.006 \\
(0.004)\end{array}$ & $\begin{array}{c}0.227^{* * *} \\
(0.058)\end{array}$ & $\begin{array}{c}0.152^{* *} \\
(0.061)\end{array}$ & $\begin{array}{c}0.150^{* *} \\
(0.062)\end{array}$ & $\begin{array}{c}0.111^{* *} \\
(0.047)\end{array}$ & $\begin{array}{c}0.136^{*+*} \\
(0.049)\end{array}$ & $\begin{array}{c}0.114^{* *} \\
(0.048)\end{array}$ \\
\hline Log population & $\begin{array}{c}0.031^{* *} \\
(0.006)\end{array}$ & $\begin{array}{c}0.032 * * * \\
(0.005)\end{array}$ & $\begin{array}{c}0.032 * * * * \\
(0.005)\end{array}$ & $\begin{array}{c}0.029^{* * * *} \\
(0.005)\end{array}$ & $\begin{array}{c}0.034^{* * *} \\
(0.005)\end{array}$ & $\begin{array}{c}0.028 * * * \\
(0.005)\end{array}$ & $\begin{array}{c}0.061^{* * *} \\
(0.015)\end{array}$ & $\begin{array}{c}0.077^{* * *} \\
(0.015)\end{array}$ & $\begin{array}{c}0.078^{* * *} \\
(0.015)\end{array}$ & $\begin{array}{c}0.076^{* * *} \\
(0.015)\end{array}$ & $\begin{array}{c}0.094^{* * *} \\
(0.016)\end{array}$ & $\begin{array}{c}0.085^{* * *} \\
(0.018)\end{array}$ \\
\hline Market acoess & $\begin{array}{c}0.299^{* * *} \\
(0.067)\end{array}$ & $\begin{array}{c}0.330^{* * *} \\
(0.048)\end{array}$ & $\begin{array}{c}0.326^{* * *} \\
(0.049)\end{array}$ & $\begin{array}{c}0.316^{* * *} \\
(0.052)\end{array}$ & $\begin{array}{c}0.316^{* * *} \\
(0.051)\end{array}$ & $\begin{array}{c}0.306^{* * *} \\
(0.052)\end{array}$ & $\begin{array}{l}0.656^{*} \\
(0.390)\end{array}$ & $\begin{array}{c}0.433 \\
(0.362)\end{array}$ & $\begin{array}{c}0.464 \\
(0.365)\end{array}$ & $\begin{array}{c}0.423 \\
(0.360)\end{array}$ & $\begin{array}{c}0.354 \\
(0.363)\end{array}$ & $\begin{array}{c}0.466 \\
(0.356)\end{array}$ \\
\hline Secondary education & $\begin{array}{c}0.002^{* * *} \\
(0.001)\end{array}$ & $\begin{array}{c}0.002^{* * *} \\
(0.000)\end{array}$ & $\begin{array}{c}0.002^{* * * *} \\
(0.000)\end{array}$ & $\begin{array}{c}0.001^{* *} \\
(0.000)\end{array}$ & $\begin{array}{c}0.002^{*+*} \\
(0.000)\end{array}$ & $\begin{array}{c}0.001^{* * *} \\
(0.000)\end{array}$ & $\begin{array}{c}0.009^{* * *} \\
(0.002)\end{array}$ & $\begin{array}{c}0.008 * * * \\
(0.002)\end{array}$ & $\begin{array}{c}0.008^{* * *} \\
(0.002)\end{array}$ & $\begin{array}{c}0.006^{* * *} \\
(0.002)\end{array}$ & $\begin{array}{c}0.007^{* * * *} \\
(0.002)\end{array}$ & $\begin{array}{c}0.007^{* * *} \\
(0.002)\end{array}$ \\
\hline Log REER index & $\begin{array}{c}-0.137^{* * * *} \\
(0.030)\end{array}$ & & & & & & $\begin{array}{c}-0.317^{* * *} \\
(0.077)\end{array}$ & & & & & \\
\hline Log RER & & $\begin{array}{c}-0.072 * * * \\
(0.026)\end{array}$ & & & & & & $\begin{array}{l}-0.166^{*} \\
(0.086)\end{array}$ & & & & \\
\hline RER misalignment & & & $\begin{array}{c}-0.056^{* *} \\
(0.024)\end{array}$ & & & & & & $\begin{array}{c}-0.128^{*} \\
(0.074)\end{array}$ & & & \\
\hline Theil index & & & & $\begin{array}{c}-0.025 * * * \\
(0.009)\end{array}$ & & & & & & $\begin{array}{c}-0.045 \\
(0.036)\end{array}$ & & \\
\hline Theil, extensive margin & & & & & $\begin{array}{l}-0.005 \\
(0.017)\end{array}$ & & & & & & $\begin{array}{c}-0.140^{* *} \\
(0.063)\end{array}$ & \\
\hline Theil, intensive margin & & & & & & $\begin{array}{c}-0.031^{* * * *} \\
(0.010)\end{array}$ & & & & & & $\begin{array}{c}0.008 \\
(0.042)\end{array}$ \\
\hline Observations & 1,111 & 1,856 & 1,856 & 1,854 & 1,845 & 1,848 & 193 & 275 & 275 & 293 & 292 & 293 \\
\hline Countries & 91 & 155 & 155 & 161 & 161 & 161 & 21 & 28 & 28 & 30 & 30 & 30 \\
\hline Accelerations included & $69 / 175$ & $105 / 175$ & $105 / 175$ & $109 / 175$ & $109 / 175$ & $109 / 175$ & $19 / 38$ & $23 / 38$ & $23 / 38$ & $25 / 38$ & $25 / 38$ & $25 / 38$ \\
\hline Year FE & Yes & Yes & Yes & Yes & Yes & Yes & Yes & Yes & Yes & Yes & Yes & Yes \\
\hline Sample & World & World & World & World & World & World & LAC & LAC & LAC & $\mathrm{LAC}$ & LAC & LAC \\
\hline McFadden $\mathrm{R}^{2}$ & 0.158 & 0.149 & 0.147 & 0.134 & 0.129 & 0.134 & 0.317 & 0.247 & 0.243 & 0.221 & 0.23 & 0.217 \\
\hline Pseudo $\mathrm{R}^{2}$ & 0.129 & 0.119 & 0.118 & 0.111 & 0.107 & 0.111 & 0.266 & 0.219 & 0.216 & 0.2 & 0.207 & 0.197 \\
\hline Observed $\%$ of $\mathrm{EA}=1$ & 17.82 & 16.92 & 16.92 & 17.64 & 17.67 & 17.70 & 25.91 & 25.46 & 25.46 & 25.26 & 25.34 & 25.26 \\
\hline \% correctly classified & 68.05 & 67.46 & 67.19 & 66.29 & 65.96 & 65.86 & 73.58 & 73.46 & 73.09 & 70.31 & 72.95 & 69.28 \\
\hline Panel B: Services & $(1)$ & $(2)$ & (3) & (4) & $(5)$ & $(6)$ & $\overline{(7)}$ & $(8)$ & $(9)$ & $\overline{(10)}$ & $\begin{array}{l}(11) \\
\end{array}$ & $(12)$ \\
\hline Log real GDP cap. & $\begin{array}{c}-0.090 \\
(0.103)\end{array}$ & $\begin{array}{c}-0.184^{* *} \\
(0.079)\end{array}$ & $\begin{array}{c}-0.186^{* *} \\
(0.080)\end{array}$ & $\begin{array}{c}-0.231^{* * *} \\
(0.084)\end{array}$ & $\begin{array}{c}-0.211^{\bullet} \\
(0.083)\end{array}$ & $\begin{array}{c}-0.200^{* *} \\
(0.084)\end{array}$ & $\begin{array}{l}-1.353 \\
(0.909)\end{array}$ & $\begin{array}{c}0.281 \\
(0.812)\end{array}$ & $\begin{array}{c}0.245 \\
(0.810)\end{array}$ & $\begin{array}{l}-0.077 \\
(0.587)\end{array}$ & $\begin{array}{l}-0.212 \\
(0.664)\end{array}$ & $\begin{array}{l}-0.142 \\
(0.540)\end{array}$ \\
\hline Log real GDP cap. ${ }^{2}$ & $\begin{array}{c}0.004 \\
(0.006)\end{array}$ & $\begin{array}{l}0.011^{* *} \\
(0.005)\end{array}$ & $\begin{array}{c}0.010^{* *} \\
(0.005)\end{array}$ & $\begin{array}{c}0.013^{* * *} \\
(0.005)\end{array}$ & $\begin{array}{l}0.012 * * \\
(0.005)\end{array}$ & $\begin{array}{l}0.011^{* *} \\
(0.005)\end{array}$ & $\begin{array}{c}0.066 \\
(0.053)\end{array}$ & $\begin{array}{c}-0.024 \\
(0.047)\end{array}$ & $\begin{array}{c}-0.023 \\
(0.047)\end{array}$ & $\begin{array}{c}-0.004 \\
(0.035)\end{array}$ & $\begin{array}{c}0.003 \\
(0.039)\end{array}$ & $\begin{array}{c}0.001 \\
(0.032)\end{array}$ \\
\hline Log population & $\begin{array}{c}0.029 * * * \\
(0.006)\end{array}$ & $\begin{array}{c}0.032^{* * *} \\
(0.005)\end{array}$ & $\begin{array}{c}0.032 * * * \\
(0.005)\end{array}$ & $\begin{array}{c}0.028^{* * *} \\
(0.006)\end{array}$ & $\begin{array}{c}0.034^{* *} \\
(0.005)\end{array}$ & $\begin{array}{c}0.030 * * * \\
(0.005)\end{array}$ & $\begin{array}{c}0.054^{* * *} \\
(0.020)\end{array}$ & $\begin{array}{c}0.043^{*+*} \\
(0.013)\end{array}$ & $\begin{array}{c}0.043^{* * *} \\
(0.013)\end{array}$ & $\begin{array}{c}0.018 \\
(0.014)\end{array}$ & $\begin{array}{c}0.055^{*+*} \\
(0.013)\end{array}$ & $\begin{array}{c}0.016 \\
(0.016)\end{array}$ \\
\hline Market access & $\begin{array}{c}-0.047 \\
(0.070)\end{array}$ & $\begin{array}{c}0.047 \\
(0.056)\end{array}$ & $\begin{array}{c}0.045 \\
(0.056)\end{array}$ & $\begin{array}{c}0.036 \\
(0.058)\end{array}$ & $\begin{array}{c}0.042 \\
(0.058)\end{array}$ & $\begin{array}{c}0.027 \\
(0.058)\end{array}$ & $\begin{array}{c}-0.695 \\
(0.520)\end{array}$ & $\begin{array}{c}-0.406 \\
(0.388)\end{array}$ & $\begin{array}{c}-0.383 \\
(0.393)\end{array}$ & $\begin{array}{c}-0.361 \\
(0.279)\end{array}$ & $\begin{array}{c}-0.604^{*} \\
(0.309)\end{array}$ & $\begin{array}{c}-0.301 \\
(0.331)\end{array}$ \\
\hline Secondary education & $\begin{array}{l}0.002{ }^{* *} \\
(0.001)\end{array}$ & $\begin{array}{c}0.002^{* * *} \\
(0.001)\end{array}$ & $\begin{array}{c}0.002^{* * *} \\
(0.001)\end{array}$ & $\begin{array}{l}0.001 * * \\
(0.001)\end{array}$ & $\begin{array}{l}0.001^{* *} \\
(0.001)\end{array}$ & $\begin{array}{l}0.001 * * \\
(0.001)\end{array}$ & $\begin{array}{l}0.004^{*} \\
(0.003)\end{array}$ & $\begin{array}{c}0.002 \\
(0.002)\end{array}$ & $\begin{array}{c}0.002 \\
(0.002)\end{array}$ & $\begin{array}{l}-0.001 \\
(0.002)\end{array}$ & $\begin{array}{c}0.001 \\
(0.002)\end{array}$ & $\begin{array}{c}-0.001 \\
(0.002)\end{array}$ \\
\hline Log REER index & $\begin{array}{c}-0.120^{* * *} \\
(0.041)\end{array}$ & & & & & & $\begin{array}{c}-0.211^{*} \\
(0.110)\end{array}$ & & & & & \\
\hline Log RER & & $\begin{array}{c}-0.055^{* *} \\
(0.027)\end{array}$ & & & & & & $\begin{array}{c}-0.089 \\
(0.067)\end{array}$ & & & & \\
\hline RER misalignment & & & $\begin{array}{l}-0.047^{*} \\
(0.025)\end{array}$ & & & & & & $\begin{array}{c}-0.077 \\
(0.061)\end{array}$ & & & \\
\hline Theil index & & & & $\begin{array}{c}-0.026^{* *} \\
(0.010)\end{array}$ & & & & & & $\begin{array}{c}-0.117^{* * *} \\
(0.033)\end{array}$ & & \\
\hline Theil, extensive margin & & & & & $\begin{array}{c}-0.032^{*} \\
(0.019)\end{array}$ & & & & & & $\begin{array}{l}-0.057 \\
(0.045)\end{array}$ & \\
\hline Theil, intensive margin & & & & & & $\begin{array}{l}-0.021^{*} \\
(0.012)\end{array}$ & & & & & & $\begin{array}{c}-0.113^{* * * *} \\
(0.041)\end{array}$ \\
\hline Observations & 908 & 1,508 & 1,508 & 1,504 & 1,500 & 1,501 & 133 & 237 & 237 & 250 & 250 & 250 \\
\hline Countries & 92 & 155 & 155 & 160 & 160 & 160 & 21 & 27 & 27 & 29 & 29 & 29 \\
\hline Accelerations included & $58 / 162$ & $90 / 162$ & $90 / 162$ & $95 / 162$ & $95 / 162$ & $95 / 162$ & $8 / 26$ & $13 / 26$ & $13 / 26$ & $15 / 26$ & $15 / 26$ & $15 / 26$ \\
\hline Year FE & Yes & Yes & Yes & Yes & Yes & Yes & Yes & Yes & Yes & Yes & Yes & Yes \\
\hline Sample & World & World & World & World & World & World & LAC & LAC & LAC & LAC & LAC & LAC \\
\hline McFadden $\mathrm{R}^{2}$ & 0.205 & 0.168 & 0.168 & 0.169 & 0.16 & 0.161 & 0.265 & 0.21 & 0.208 & 0.231 & 0.216 & 0.231 \\
\hline Pseudo $R^{2}$ & 0.169 & 0.139 & 0.139 & 0.142 & 0.136 & 0.137 & 0.211 & 0.156 & 0.154 & 0.176 & 0.167 & 0.176 \\
\hline Observed $\%$ of $\mathrm{EA}=1$ & 19.60 & 18.57 & 18.57 & 19.35 & 19.33 & 19.39 & 18.80 & 16.03 & 16.03 & 17.60 & 17.60 & 17.60 \\
\hline$\%$ correctly classified & 72.36 & 69.89 & 69.70 & 69.68 & 69.67 & 69.55 & 69.17 & 66.25 & 66.25 & 70.80 & 70.00 & 70.00 \\
\hline
\end{tabular}




\begin{tabular}{|c|c|c|c|c|c|c|c|c|c|c|c|c|}
\hline Panel A: Goods & $(1)$ & $(2)$ & $(3)$ & (4) & $(5)$ & (6) & $(7)$ & (8) & (9) & (10) & $(11)$ & $(12)$ \\
\hline Log real GDP cap. & $\begin{array}{c}0.168^{* * *} \\
(0.065)\end{array}$ & $\begin{array}{c}0.064 \\
(0.113)\end{array}$ & $\begin{array}{c}0.069 \\
(0.113)\end{array}$ & $\begin{array}{c}0.044 \\
(0.112)\end{array}$ & $\begin{array}{l}0.143^{*} \\
(0.076)\end{array}$ & $\begin{array}{l}0.165^{* *} \\
(0.071)\end{array}$ & $\begin{array}{c}-1.967^{* *} \\
(0.849)\end{array}$ & $\begin{array}{c}-1.822 \\
(1.133)\end{array}$ & $\begin{array}{l}-1.799^{*} \\
(1.064)\end{array}$ & $\begin{array}{l}-2.547^{*} \\
(1.505)\end{array}$ & $\begin{array}{c}-2.290^{* *} \\
(1.076)\end{array}$ & $\begin{array}{c}-2.464^{* *} \\
(1.033)\end{array}$ \\
\hline Log real GDP cap. ${ }^{2}$ & $\begin{array}{c}-0.010^{* *} \\
(0.004)\end{array}$ & $\begin{array}{l}-0.005 \\
(0.007)\end{array}$ & $\begin{array}{l}-0.005 \\
(0.007)\end{array}$ & $\begin{array}{l}-0.003 \\
(0.007)\end{array}$ & $\begin{array}{l}-0.008^{*} \\
(0.005)\end{array}$ & $\begin{array}{c}-0.009^{* *} \\
(0.004)\end{array}$ & $\begin{array}{l}0.113^{* *} \\
(0.050)\end{array}$ & $\begin{array}{c}0.106 \\
(0.066)\end{array}$ & $\begin{array}{l}0.104^{*} \\
(0.062)\end{array}$ & $\begin{array}{l}0.151^{*} \\
(0.089)\end{array}$ & $\begin{array}{l}0.136^{* *} \\
(0.064)\end{array}$ & $\begin{array}{l}0.145^{* *} \\
(0.061)\end{array}$ \\
\hline Log population & $\begin{array}{c}0.034^{* * *} \\
(0.004)\end{array}$ & $\begin{array}{c}0.023 * * * \\
(0.007)\end{array}$ & $\begin{array}{c}0.023^{* * *} \\
(0.007)\end{array}$ & $\begin{array}{c}0.022^{* * *} \\
(0.007)\end{array}$ & $\begin{array}{c}0.041^{* * *} \\
(0.006)\end{array}$ & $\begin{array}{c}0.044^{* * *} \\
(0.005)\end{array}$ & $\begin{array}{c}0.076^{* * *} \\
(0.016)\end{array}$ & $\begin{array}{l}-0.007 \\
(0.014)\end{array}$ & $\begin{array}{l}-0.006 \\
(0.014)\end{array}$ & $\begin{array}{l}-0.017 \\
(0.014)\end{array}$ & $\begin{array}{c}0.061^{* * *} \\
(0.019)\end{array}$ & $\begin{array}{c}0.061^{* * *} \\
(0.020)\end{array}$ \\
\hline Market acosss & $\begin{array}{c}0.298^{* * *} \\
(0.050)\end{array}$ & $\begin{array}{c}0.249^{* * *} \\
(0.088)\end{array}$ & $\begin{array}{c}0.248^{* * *} \\
(0.088)\end{array}$ & $\begin{array}{c}0.249^{* * *} \\
(0.087)\end{array}$ & $\begin{array}{c}0.393^{* * *} \\
(0.060)\end{array}$ & $\begin{array}{c}0.348^{* * *} \\
(0.055)\end{array}$ & $\begin{array}{c}0.473 \\
(0.410)\end{array}$ & $\begin{array}{c}3.496^{* * *} \\
(0.941)\end{array}$ & $\begin{array}{c}3.604^{* * *} \\
(0.929)\end{array}$ & $\begin{array}{c}2.517^{* * *} \\
(0.855)\end{array}$ & $\begin{array}{c}1.438^{* * *} \\
(0.507)\end{array}$ & $\begin{array}{c}1.484^{* * * *} \\
(0.481)\end{array}$ \\
\hline Secondary education & $\begin{array}{c}0.002^{* * *} \\
(0.000)\end{array}$ & $\begin{array}{c}0.001 \\
(0.001)\end{array}$ & $\begin{array}{l}0.001 \\
(0.001)\end{array}$ & $\begin{array}{c}0.001 \\
(0.001)\end{array}$ & $\begin{array}{c}0.002^{* * *} \\
(0.001)\end{array}$ & $\begin{array}{c}0.002^{* * *} \\
(0.000)\end{array}$ & $\begin{array}{c}0.006^{* * *} \\
(0.002)\end{array}$ & $\begin{array}{l}-0.000 \\
(0.001)\end{array}$ & $\begin{array}{c}0.000 \\
(0.001)\end{array}$ & $\begin{array}{c}0.000 \\
(0.001)\end{array}$ & $\begin{array}{c}0.011^{* * *} \\
(0.002)\end{array}$ & $\begin{array}{c}0.011^{* * * *} \\
(0.002)\end{array}$ \\
\hline$\Delta$ Trade openness & $\begin{array}{c}0.044 \\
(0.028)\end{array}$ & & & & & & $\begin{array}{l}0.252^{*} \\
(0.129)\end{array}$ & & & & & \\
\hline Tariffs & & $\begin{array}{c}-0.006^{* *} \\
(0.003)\end{array}$ & & & & & & $\begin{array}{c}-0.001 \\
(0.008)\end{array}$ & & & & \\
\hline Tariffs, manufactures & & & $\begin{array}{c}-0.006^{* *} \\
(0.003)\end{array}$ & & & & & & $\begin{array}{c}0.003 \\
(0.008)\end{array}$ & & & \\
\hline Tariffs, primary products & & & & $\begin{array}{c}-0.002 \\
(0.002)\end{array}$ & & & & & & $\begin{array}{l}-0.015 \\
(0.010)\end{array}$ & & \\
\hline Agriculture index & & & & & $\begin{array}{l}0.060^{* *} \\
(0.026)\end{array}$ & & & & & & $\begin{array}{l}0.214^{* *} \\
(0.088)\end{array}$ & \\
\hline Electricity \& Telecoms & & & & & & $\begin{array}{c}-0.116^{* *} \\
(0.045)\end{array}$ & & & & & & $\begin{array}{c}0.024 \\
(0.178) \\
\end{array}$ \\
\hline Observations & 1,901 & 711 & 711 & 711 & 1,379 & 1,509 & 278 & 98 & 98 & 98 & 210 & 225 \\
\hline Countries & 167 & 140 & 140 & 140 & 120 & 126 & 30 & 26 & 26 & 26 & 23 & 24 \\
\hline Acoelerations included & $107 / 175$ & $40 / 175$ & $40 / 175$ & $40 / 175$ & $86 / 175$ & $92 / 175$ & $25 / 38$ & $8 / 38$ & $8 / 38$ & $8 / 38$ & $24 / 38$ & $25 / 38$ \\
\hline Year FE & Yes & Yes & Yes & $\mathrm{Y}_{\mathrm{es}}$ & Yes & Yes & Yess & Yes & Yes & Yes & $\mathrm{Y}_{\mathrm{es}}$ & Yes \\
\hline Sample & World & World & World & World & World & World & LAC & $\mathrm{LAC}$ & LAC & LAC & LAC & $\mathrm{LAC}$ \\
\hline McFadden $\mathrm{R}^{2}$ & 0.144 & 0.130 & 0.131 & 0.123 & 0.180 & 0.176 & 0.228 & 0.360 & 0.361 & 0.418 & 0.283 & 0.268 \\
\hline Pseudo $R^{2}$ & 0.116 & 0.105 & 0.106 & 0.100 & 0.147 & 0.143 & 0.209 & 0.277 & 0.278 & 0.308 & 0.260 & 0.249 \\
\hline Observed $\%$ of $\mathrm{EA}=1$ & 16.83 & 16.74 & 16.74 & 16.74 & 18.49 & 18.16 & 26.62 & 22.45 & 22.45 & 22.45 & 31.43 & 30.67 \\
\hline$\%$ correctly classified & 66.75 & 67.09 & 66.53 & 65.12 & 69.83 & 68.66 & 70.86 & 79.59 & 82.65 & 83.67 & 72.86 & 70.67 \\
\hline Panel B: Services & (1) & $(2)$ & $(3)$ & (4) & $(5)$ & 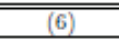 & $(7)$ & $(8)$ & $(9)$ & $(10)$ & (11) & (12) \\
\hline Log real GDP cap. & $\begin{array}{l}-0.045 \\
(0.077)\end{array}$ & $\begin{array}{l}-0.135 \\
(0.190)\end{array}$ & $\begin{array}{l}-0.134 \\
(0.190)\end{array}$ & $\begin{array}{l}-0.158 \\
(0.190)\end{array}$ & $\begin{array}{c}-0.202^{* *} \\
(0.095)\end{array}$ & $\begin{array}{c}-0.112 \\
(0.094)\end{array}$ & $\begin{array}{c}0.328 \\
(0.685)\end{array}$ & $\begin{array}{l}-1.299 \\
(1.190)\end{array}$ & $\begin{array}{l}-1.421 \\
(1.193)\end{array}$ & $\begin{array}{c}0.215 \\
(0.604)\end{array}$ & $\begin{array}{l}-0.179 \\
(0.889)\end{array}$ & $\begin{array}{l}-1.340^{*} \\
(0.714)\end{array}$ \\
\hline Log real GDP cap. ${ }^{2}$ & $\begin{array}{c}0.002 \\
(0.005)\end{array}$ & $\begin{array}{c}0.006 \\
(0.011)\end{array}$ & $\begin{array}{c}0.006 \\
(0.011)\end{array}$ & $\begin{array}{l}0.007 \\
(0.011)\end{array}$ & $\begin{array}{l}0.010^{*} \\
(0.006)\end{array}$ & $\begin{array}{c}0.005 \\
(0.005)\end{array}$ & $\begin{array}{l}-0.028 \\
(0.040)\end{array}$ & $\begin{array}{c}0.067 \\
(0.070)\end{array}$ & $\begin{array}{c}0.074 \\
(0.070)\end{array}$ & $\begin{array}{l}-0.020 \\
(0.036)\end{array}$ & $\begin{array}{l}-0.001 \\
(0.052)\end{array}$ & $\begin{array}{l}0.068^{*} \\
(0.041)\end{array}$ \\
\hline Log population & $\begin{array}{c}0.033^{* * *} \\
(0.005)\end{array}$ & $\begin{array}{c}0.043^{* * *} \\
(0.011)\end{array}$ & $\begin{array}{c}0.044^{* * *} \\
(0.011)\end{array}$ & $\begin{array}{c}0.041^{* * *} \\
(0.011)\end{array}$ & $\begin{array}{c}0.035^{* * *} \\
(0.007)\end{array}$ & $\begin{array}{c}0.030^{* * *} \\
(0.007)\end{array}$ & $\begin{array}{c}0.037^{* * 8} \\
(0.012)\end{array}$ & $\begin{array}{c}0.103^{* * *} \\
(0.032)\end{array}$ & $\begin{array}{c}0.104^{* * *} \\
(0.033)\end{array}$ & $\begin{array}{c}0.064^{* * *} \\
(0.023)\end{array}$ & $\begin{array}{c}0.045^{* * *} \\
(0.015)\end{array}$ & $\begin{array}{l}0.035^{* *} \\
(0.018)\end{array}$ \\
\hline Market acoess & $\begin{array}{l}-0.008 \\
(0.054)\end{array}$ & $\begin{array}{l}-0.059 \\
(0.122)\end{array}$ & $\begin{array}{l}-0.062 \\
(0.121)\end{array}$ & $\begin{array}{l}-0.038 \\
(0.122)\end{array}$ & $\begin{array}{c}0.087 \\
(0.064)\end{array}$ & $\begin{array}{c}0.014 \\
(0.063)\end{array}$ & $\begin{array}{l}-0.227 \\
(0.295)\end{array}$ & $\begin{array}{c}-2.525^{* *} \\
(1.175)\end{array}$ & $\begin{array}{c}-2.383^{* *} \\
(1.193)\end{array}$ & $\begin{array}{c}-2.478^{* * *} \\
(0.876)\end{array}$ & $\begin{array}{c}-0.088 \\
(0.365)\end{array}$ & $\begin{array}{l}-0.494 \\
(0.408)\end{array}$ \\
\hline Secondary education & $\begin{array}{c}0.002^{* * *} \\
(0.001)\end{array}$ & $\begin{array}{l}0.003^{* *} \\
(0.001)\end{array}$ & $\begin{array}{l}0.003^{* * *} \\
(0.001)\end{array}$ & $\begin{array}{l}0.003^{* *} \\
(0.001)\end{array}$ & $\begin{array}{c}0.002^{* * *} \\
(0.001)\end{array}$ & $\begin{array}{l}0.002^{* *} \\
(0.001)\end{array}$ & $\begin{array}{c}0.001 \\
(0.002)\end{array}$ & $\begin{array}{c}0.003 \\
(0.003)\end{array}$ & $\begin{array}{c}0.003 \\
(0.003)\end{array}$ & $\begin{array}{l}0.002^{*} \\
(0.001)\end{array}$ & $\begin{array}{c}0.002 \\
(0.002)\end{array}$ & $\begin{array}{l}0.005^{* *} \\
(0.002)\end{array}$ \\
\hline$\Delta$ Trade openness & $\begin{array}{c}0.043 \\
(0.031)\end{array}$ & & & & & & $\begin{array}{c}0.273^{* * *} \\
(0.094)\end{array}$ & & & & & \\
\hline Tariffs & & $\begin{array}{c}-0.004 \\
(0.005)\end{array}$ & & & & & & $\begin{array}{l}-0.020^{*} \\
(0.011)\end{array}$ & & & & \\
\hline Tariffs, manufactures & & & $\begin{array}{l}-0.004 \\
(0.004)\end{array}$ & & & & & & $\begin{array}{l}-0.018 \\
(0.011)\end{array}$ & & & \\
\hline Tariffs, primary products & & & & $\begin{array}{c}-0.006 \\
(0.004)\end{array}$ & & & & & & $\begin{array}{c}-0.023^{* * *} \\
(0.008)\end{array}$ & & \\
\hline Agriculture index & & & & & $\begin{array}{c}0.102^{* * *} \\
(0.031)\end{array}$ & & & & & & $\begin{array}{c}0.296^{* * *} \\
(0.069)\end{array}$ & \\
\hline Electricity \& Telecoms & & & & & & $\begin{array}{c}0.173^{* * *} \\
(0.059)\end{array}$ & & & & & & $\begin{array}{c}0.568^{* * * *} \\
(0.145)\end{array}$ \\
\hline Observations & 1,542 & 577 & 577 & 577 & 1,119 & 1,205 & 242 & 108 & 108 & 108 & 185 & 194 \\
\hline Countries & 165 & 134 & 134 & 134 & 118 & 123 & 29 & 27 & 27 & 27 & 22 & 23 \\
\hline Acoelerations included & $93 / 162$ & $57 / 162$ & $57 / 162$ & $57 / 162$ & $74 / 162$ & $78 / 162$ & $15 / 26$ & $8 / 26$ & $8 / 26$ & $8 / 26$ & $13 / 26$ & $13 / 26$ \\
\hline Year FE & Yess & Yes & Yes & $Y_{\text {es }}$ & Yes & Yes & Yess & Yes & Yess & Yes & Yess & $Y_{e s}$ \\
\hline Sample & World & World & World & World & World & World & LAC & LAC & LAC & LAC & LAC & LAC \\
\hline McFadden $\mathrm{R}^{2}$ & 0.166 & 0.147 & 0.147 & 0.150 & 0.187 & 0.158 & 0.246 & 0.258 & 0.252 & 0.340 & 0.335 & 0.272 \\
\hline Psendo $\mathrm{R}^{2}$ & 0.138 & 0.150 & 0.150 & 0.152 & 0.160 & 0.137 & 0.187 & 0.214 & 0.211 & 0.265 & 0.254 & 0.215 \\
\hline Observed $\%$ of $E A=1$ & 18.81 & 28.77 & 28.77 & 28.77 & 20.82 & 20.33 & 17.77 & 22.22 & 22.22 & 22.22 & 20.54 & 20.10 \\
\hline$\%$ correctly classified & 69.59 & 68.46 & 68.28 & 69.32 & 72.30 & 70.37 & 71.49 & 73.15 & 73.15 & 75.93 & 75.68 & 72.17 \\
\hline
\end{tabular}


33. Structural reforms geared towards boosting market competition have a bearing on the initiation of export accelerations. Agricultural reforms positively correlate with the timing of both types of export surges, irrespective of the sample considered, although results for LAC are strikingly larger in size and more significant. A one-unit rise in the index leads to a 21.4 and 29.6 percentage point increase in the likelihood of experiencing an export acceleration in goods and services, respectively. Meanwhile the corresponding figures are almost six times smaller and stand at 6 and 10.2 percentage points for the overall sample. Liberalization of telecommunication and electricity markets has a positive effect on export initiations only in the case of services, with a coefficient that is more than three times larger for LAC countries. By raising market competition through an ease of foreign entry into the domestic market, liberalization goes hand in hand with a removal of barriers to trade in services (Sáez et al., 2015). Our result is in line with the literature on the direct effects of services liberalization on firms in deregulated sectors (Lanau and Topalova, 2016) but does not support the idea of positive spillovers on downstream firms producing and exporting goods (Arnold et al., 2008, 2011, 2016). ${ }^{24}$

\section{Evidence on the link between financial reforms and exports is mixed (Table 4).}

Domestic financial liberalization does not seem to have a bearing on goods export accelerations but predicts accelerations of services exports in LAC: a one-unit increase in the aggregate index translates in a 65.6 percentage point increase in the likelihood of observing a services acceleration episode. This result is driven by the positive effect of banking sector reforms as the coefficient on the securities reform index is not statistically significant.

Although not significant at the conventional levels, capital account openness indicators are all negatively associated with the timing of goods export accelerations in Panel A, possibly reflecting the adverse effects of unfettered financial liberalization on exports via higher vulnerability to crisis risk (Rancière et al., 2008; Rancière and Tornell, 2015). However, a reversal of sign occurs for the LAC sample. Specifically, a one-unit rise in the capital account openness index leads to a 39.4 percentage point increase in the probability of experiencing a goods export acceleration. The removal of capital movement restrictions for both residents and non-residents contribute to the positive effect. In the case of services, Panel B provides limited evidence of the acceleration-triggering effect of capital account liberalization, as only the coefficient on the sub-component pertaining to the removal of restrictions on non-residents seem to be positive and statistically significant (col. 12).

\footnotetext{
24 The relationship even turns negative in the world sample.
} 


\begin{tabular}{|c|c|c|c|c|c|c|c|c|c|c|c|c|}
\hline Panel A: Goods & (1) & $(2)$ & (3) & (4) & $(5)$ & (6) & $(7)$ & $(8)$ & (9) & (10) & (11) & $(12)$ \\
\hline Log real GDP cap. & $\begin{array}{c}0.249^{* *} \\
(0.099)\end{array}$ & $\begin{array}{c}0.252^{* * *} \\
(0.099)\end{array}$ & $\begin{array}{c}0.260 * * * * \\
(0.100)\end{array}$ & $\begin{array}{l}0.145^{*} \\
(0.080)\end{array}$ & $\begin{array}{c}0.161 * * \\
(0.080)\end{array}$ & $\begin{array}{l}0.145^{*} \\
(0.080)\end{array}$ & $\begin{array}{l}-2.938^{*} \\
(1.523)\end{array}$ & $\begin{array}{l}-2.931^{*} \\
(1.525)\end{array}$ & $\begin{array}{l}-2.770^{*} \\
(1.514)\end{array}$ & $\begin{array}{c}-3.649 * * * \\
(1.234)\end{array}$ & $\begin{array}{c}-3.328 * * * \\
(1.196)\end{array}$ & $\begin{array}{r}-3.629 * * * \\
(1.239)\end{array}$ \\
\hline Log real GDP cap. ${ }^{2}$ & $\begin{array}{c}-0.015^{* * *} \\
(0.006)\end{array}$ & $\begin{array}{c}-0.015^{* *} \\
(0.006)\end{array}$ & $\begin{array}{c}-0.016 * * * \\
(0.006)\end{array}$ & $\begin{array}{c}-0.010^{* *} \\
(0.005)\end{array}$ & $\begin{array}{c}-0.010^{* *} \\
(0.005)\end{array}$ & $\begin{array}{c}-0.010^{* *} \\
(0.005)\end{array}$ & $\begin{array}{l}0.164^{*} \\
(0.091)\end{array}$ & $\begin{array}{l}0.164^{*} \\
(0.091)\end{array}$ & $\begin{array}{l}0.155^{*} \\
(0.090)\end{array}$ & $\begin{array}{c}0.209^{* \cdots} \\
(0.074)\end{array}$ & $\begin{array}{c}0.190^{* * *} \\
(0.072)\end{array}$ & $\begin{array}{c}0.207 * * * \\
(0.074)\end{array}$ \\
\hline Log population & $\begin{array}{c}0.043^{* * *} \\
(0.010)\end{array}$ & $\begin{array}{c}0.043^{* * *} \\
(0.010)\end{array}$ & $\begin{array}{c}0.042^{* * *} \\
(0.010)\end{array}$ & $\begin{array}{c}0.033 * * * \\
(0.007)\end{array}$ & $\begin{array}{c}0.034^{* * *} \\
(0.007)\end{array}$ & $\begin{array}{c}0.033 * * * \\
(0.007)\end{array}$ & $\begin{array}{l}-0.037 \\
(0.047)\end{array}$ & $\begin{array}{l}-0.037 \\
(0.047)\end{array}$ & $\begin{array}{l}-0.042 \\
(0.047)\end{array}$ & $\begin{array}{c}0.066 * * \\
(0.029)\end{array}$ & $\begin{array}{c}0.055^{* *} \\
(0.028)\end{array}$ & $\begin{array}{l}0.073 * * \\
(0.029)\end{array}$ \\
\hline Market access & $\begin{array}{c}0.416^{* * *} \\
(0.075)\end{array}$ & $\begin{array}{c}0.413^{* * *} \\
(0.075)\end{array}$ & $\begin{array}{c}0.415^{* * * *} \\
(0.076)\end{array}$ & $\begin{array}{c}0.320 * * * \\
(0.065)\end{array}$ & $\begin{array}{c}0.326^{* * *} \\
(0.065)\end{array}$ & $\begin{array}{c}0.320 * * * \\
(0.065)\end{array}$ & $\begin{array}{c}2.973 * * * \\
(0.814)\end{array}$ & $\begin{array}{c}2.939 * * * \\
(0.781)\end{array}$ & $\begin{array}{c}2.786^{* * *} \\
(0.734)\end{array}$ & $\begin{array}{l}1.246^{* *} \\
(0.525)\end{array}$ & $\begin{array}{l}1.350 * * \\
(0.532)\end{array}$ & $\begin{array}{l}1.223 * * \\
(0.523)\end{array}$ \\
\hline Secondary education & $\begin{array}{c}0.003 * \cdots * \\
(0.001)\end{array}$ & $\begin{array}{c}0.003^{* * * *} \\
(0.001)\end{array}$ & $\begin{array}{c}0.003^{* * * *} \\
(0.001)\end{array}$ & $\begin{array}{c}0.003^{* * * *} \\
(0.001)\end{array}$ & $\begin{array}{c}0.003^{* * * *} \\
(0.001)\end{array}$ & $\begin{array}{c}0.003^{* * *} \\
(0.001)\end{array}$ & $\begin{array}{c}0.017^{* * *} \\
(0.004)\end{array}$ & $\begin{array}{c}0.017^{* * *} \\
(0.004)\end{array}$ & $\begin{array}{c}0.017^{* \cdots *} \\
(0.004)\end{array}$ & $\begin{array}{c}0.013^{* \cdots *} \\
(0.003)\end{array}$ & $\begin{array}{c}0.012^{* * *} \\
(0.003)\end{array}$ & $\begin{array}{c}0.013 * * * \\
(0.003)\end{array}$ \\
\hline Dornestic financial liberalization & $\begin{array}{l}-0.081 \\
(0.089)\end{array}$ & & & & & & $\begin{array}{l}-0.253 \\
(0.337)\end{array}$ & & & & & \\
\hline Banking sector reform & & $\begin{array}{c}-0.096 \\
(0.084)\end{array}$ & & & & & & $\begin{array}{c}-0.257 \\
(0.325)\end{array}$ & & & & \\
\hline Securities market reform & & & $\begin{array}{c}0.030 \\
(0.056)\end{array}$ & & & & & & $\begin{array}{c}-0.011 \\
(0.198)\end{array}$ & & & \\
\hline Capital account operniness & & & & $\begin{array}{l}-0.059 \\
(0.045)\end{array}$ & & & & & & $\begin{array}{c}0.454 * * * \\
(0.156)\end{array}$ & & \\
\hline Capital acc. openness, residents & & & & & $\begin{array}{l}-0.065 \\
(0.040)\end{array}$ & & & & & & $\begin{array}{c}0.3883^{* * * *} \\
(0.137)\end{array}$ & \\
\hline Cap. acc. openness, non-residents & & & & & & $\begin{array}{l}-0.040 \\
(0.042)\end{array}$ & & & & & & $\begin{array}{c}0.401 * * * \\
(0.148)\end{array}$ \\
\hline Observations & 1,025 & 1,025 & 1,025 & 1,345 & 1,367 & 1,345 & 155 & 155 & $15 K$ & 211 & 211 & 211 \\
\hline Countries & 88 & 88 & 88 & 114 & 115 & 114 & 16 & 16 & 16 & 22 & 22 & 22 \\
\hline A coelerations included & $77 / 175$ & $77 / 175$ & $77 / 175$ & $92 / 175$ & $92 / 175$ & $92 / 175$ & $22 / 38$ & $22 / 38$ & $22 / 38$ & $25 / 38$ & $25 / 38$ & $25 / 38$ \\
\hline Year FE & Yes & Yes & Yes & Yes & Yes & Yes & Yes & Yes & Yes & Yes & Yes & Yes \\
\hline Sample & World & World & World & World & World & World & LAC & LAC & LAC & LAC & $\mathrm{LAC}$ & LAC \\
\hline McFadden $\mathrm{R}^{2}$ & 0.187 & 0.187 & 0.186 & 0.158 & 0.155 & 0.157 & 0.296 & 0.296 & 0.294 & 0.226 & 0.224 & 0.219 \\
\hline Pseudo $\mathrm{R}^{2}$ & 0.165 & 0.166 & 0.165 & 0.137 & 0.134 & 0.137 & 0.282 & 0.282 & 0.281 & 0.224 & 0.222 & 0.219 \\
\hline Observed $\%$ of $\mathrm{EA}=1$ & 22.24 & 22.24 & 22.24 & 20.30 & 19.97 & 20.30 & 38.07 & 38.07 & 38.07 & 33.65 & 33.65 & 33.65 \\
\hline \% correctly classified & 69.37 & 69.17 & 67.90 & 66.69 & 66.28 & 66.84 & 71.61 & 72.90 & 72.26 & 71.09 & 72.04 & 69.19 \\
\hline Panel B: Services & (1) & (2) & (3) & (4) & (5) & (6) & (7) & $(8)$ & (9) & (10) & (11) & $\overline{(12)}$ \\
\hline Log real GDP cap. & $\begin{array}{c}-0.297^{* * *} \\
(0.129)\end{array}$ & $\begin{array}{c}-0.297^{* *} \\
(0.129)\end{array}$ & $\begin{array}{c}-0.297^{* *} \\
(0.129)\end{array}$ & $\begin{array}{c}-0.256^{* *} \\
(0.110)\end{array}$ & $\begin{array}{c}-0.245^{* *} \\
(0.109)\end{array}$ & $\begin{array}{c}-0.240^{* * *} \\
(0.113)\end{array}$ & $\begin{array}{l}-1.931 \\
(1.413)\end{array}$ & $\begin{array}{l}-1.884 \\
(1.392)\end{array}$ & $\begin{array}{c}-2.818 * * \\
(1.395)\end{array}$ & $\begin{array}{c}-0.604 \\
(0.801)\end{array}$ & $\begin{array}{l}-0.538 \\
(0.808)\end{array}$ & $\begin{array}{c}-0.639 \\
(0.786)\end{array}$ \\
\hline Log real GDP cap. ${ }^{2}$ & $\begin{array}{l}0.015^{* *} \\
(0.008)\end{array}$ & $\begin{array}{l}0.015^{* *} \\
(0.008)\end{array}$ & $\begin{array}{c}0.015^{* *} \\
(0.008)\end{array}$ & $\begin{array}{c}0.012^{*} \\
(0.007)\end{array}$ & $\begin{array}{l}0.012^{*} \\
(0.006)\end{array}$ & $\begin{array}{l}0.011^{*} \\
(0.007)\end{array}$ & $\begin{array}{c}0.098) \\
(0.083)\end{array}$ & $\begin{array}{c}0.095 \\
(0.082)\end{array}$ & $\begin{array}{l}0.151^{*} \\
(0.083)\end{array}$ & $\begin{array}{c}0.024 \\
(0.047)\end{array}$ & $\begin{array}{c}0.019 \\
(0.047)\end{array}$ & $\begin{array}{c}0.027 \\
(0.046)\end{array}$ \\
\hline Log population & $\begin{array}{l}0.021^{*} \\
(0.012)\end{array}$ & $\begin{array}{l}0.021^{*} \\
(0.012)\end{array}$ & $\begin{array}{l}0.023^{*} \\
(0.012)\end{array}$ & $\begin{array}{l}0.020^{* *} \\
(0.008)\end{array}$ & $\begin{array}{l}0.021^{* *} \\
(0.008)\end{array}$ & $\begin{array}{l}0.020^{* *} \\
(0.008)\end{array}$ & $\begin{array}{c}0.040 \\
(0.041)\end{array}$ & $\begin{array}{c}0.040 \\
(0.040)\end{array}$ & $\begin{array}{c}0.062) \\
0.062) \\
(0.041)\end{array}$ & $\begin{array}{l}0.049^{*} \\
(0.026)\end{array}$ & $\begin{array}{l}0.042^{*} \\
(0.024)\end{array}$ & $\begin{array}{l}0.060^{* *} \\
(0.028)\end{array}$ \\
\hline Market access & $\begin{array}{c}0.037 \\
(0.085)\end{array}$ & $\begin{array}{c}0.036 \\
(0.085)\end{array}$ & $\begin{array}{c}0.043 \\
(0.086)\end{array}$ & $\begin{array}{r}-0.013 \\
(0.074)\end{array}$ & $\begin{array}{c}0.001 \\
(0.074)\end{array}$ & $\begin{array}{c}-0.018 \\
(0.075)\end{array}$ & $\begin{array}{c}-0.872 \\
(0.759)\end{array}$ & $\begin{array}{l}-0.842 \\
(0.738)\end{array}$ & $\begin{array}{c}-0.737 \\
(0.856)\end{array}$ & $\begin{array}{l}-0.832^{*} \\
(0.480)\end{array}$ & $\begin{array}{l}-0.645 \\
(0.471)\end{array}$ & $\begin{array}{c}-1.041^{* *} \\
(0.480)\end{array}$ \\
\hline Secondary education & $\begin{array}{c}0.003^{* * *} \\
(0.001)\end{array}$ & $\begin{array}{c}0.003^{* * *} \\
(0.001)\end{array}$ & $\begin{array}{c}0.003^{* * *} \\
(0.001)\end{array}$ & $\begin{array}{c}0.003^{* * * *} \\
(0.001)\end{array}$ & $\begin{array}{c}0.003^{* * * *} \\
(0.001)\end{array}$ & $\begin{array}{c}0.002^{* * *} \\
(0.001)\end{array}$ & $\begin{array}{l}0.007^{*} \\
(0.004)\end{array}$ & $\begin{array}{l}0.007^{*} \\
(0.004)\end{array}$ & $\begin{array}{l}0.008^{* * *} \\
(0.004)\end{array}$ & $\begin{array}{c}0.004 \\
(0.003)\end{array}$ & $\begin{array}{c}0.004 \\
(0.003)\end{array}$ & $\begin{array}{c}0.004 \\
(0.003)\end{array}$ \\
\hline Domestic financial liberalization & $\begin{array}{c}-0.057 \\
(0.111)\end{array}$ & & & & & & $\begin{array}{l}0.656^{* *} \\
(0.282)\end{array}$ & & & & & \\
\hline Banking sector reform & & $\begin{array}{c}-0.043 \\
(0.101)\end{array}$ & & & & & & $\begin{array}{c}0.720^{* * *} \\
(0.273)\end{array}$ & & & & \\
\hline Securities market reform & & & $\begin{array}{l}-0.042 \\
(0.073)\end{array}$ & & & & & & $\begin{array}{c}-0.021 \\
(0.172)\end{array}$ & & & \\
\hline Capital sccount openness & & & & $\begin{array}{c}0.027 \\
(0.056)\end{array}$ & & & & & & $\begin{array}{c}0.223 \\
(0.152)\end{array}$ & & \\
\hline Capital acc. openness, residents & & & & & $\begin{array}{c}-0.004 \\
(0.048)\end{array}$ & & & & & & $\begin{array}{c}0.107 \\
(0.126)\end{array}$ & \\
\hline Cap. acc. openness, non-residents & & & & & & $\begin{array}{c}0.072 \\
(0.057)\end{array}$ & & & & & & $\begin{array}{l}0.322^{* * *} \\
(0.151)\end{array}$ \\
\hline Observations & 846 & 846 & 846 & 1,057 & 1,074 & 1,057 & 145 & 145 & 145 & 190 & 190 & 190 \\
\hline Countries & 87 & 87 & 87 & 112 & 113 & 112 & 16 & 16 & 16 & 21 & 21 & 21 \\
\hline Accelerations included & $66 / 162$ & $66 / 162$ & $66 / 162$ & $78 / 162$ & $79 / 162$ & $78 / 162$ & $12 / 26$ & $12 / 26$ & $12 / 26$ & $14 / 26$ & $14 / 26$ & $14 / 26$ \\
\hline Year FE & Yes & Yes & Yes & Yes & Yes & Yes & Yes & Yes & Yes & Yes & Yes & Yes \\
\hline Sample & World & World & World & World & World & World & LAC & LAC & LAC & LAC & $\mathrm{LAC}$ & LAC \\
\hline McFadden $\mathrm{R}^{2}$ & 0.155 & 0.155 & 0.155 & 0.169 & 0.167 & 0.170 & 0.208 & 0.217 & 0.180 & 0.184 & 0.175 & 0.196 \\
\hline Pseudo 2 & 0.148 & 0.148 & 0.148 & 0.154 & 0.152 & 0.155 & 0.191 & 0.198 & 0.170 & 0.163 & 0.156 & 0.172 \\
\hline Observed $\%$ of EA $=1$ & 24.82 & 24.82 & 24.82 & 22.90 & 22.81 & 22.90 & 25.52 & 25.52 & 25.52 & 22.11 & 22.11 & 22.11 \\
\hline$\%$ correctly classified & 70.69 & 70.69 & 71.28 & 73.42 & 72.63 & 73.32 & 71.72 & 74.48 & 68.97 & 67.37 & 65.26 & 64.21 \\
\hline
\end{tabular}




\section{FDI inflows, globalization, and participation in global value chains are}

associated with export surges (Table 5). A 1 percent increase in the three-year change in FDI inflows raises the probability of observing goods export accelerations by 1.2 percentage points, but the coefficient is not statistically significant for LAC. This may reflect the fact that FDI in the region is mainly channeled into the minerals sector with limited spillovers for the rest of the economy. Rather, FDI matters for raising the probability of occurrence of services export surges in LAC, consistent with Van der Marel (2012). The KOF index of globalization also positively correlates with the likelihood of a surge, especially in LAC. Furthermore, Table 5 also indicates that an increase in GVC participation is conducive to sustained export growth, raising the probability of occurrence of goods and services export accelerations by 27 and 37.7 percentage points respectively. The size of the effect is larger for LAC countries which enjoy on average an effect that is almost four to seven times larger. In the case of goods, this positive relationship transits through the benefits of backward linkages in the world sample, whereas both backward and forward participation seem to matter in LAC. In the cases of services, although the results for the world sample suggest a negative correlation between the timing of services export accelerations and growth in domestic value content, LAC countries enjoy an enhanced probability of recording an acceleration through a higher growth in the foreign value-added content of exports. 
Table 5. Correlates of Export Accelerations: Globalization and GVC Participation

\begin{tabular}{|c|c|c|c|c|c|c|c|c|c|c|c|c|}
\hline Panel A: Goods & $(1)$ & $(2)$ & $(3)$ & $(4)$ & 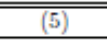 & (6) & $(7)$ & $(8)$ & 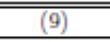 & $(10)$ & $(111)$ & 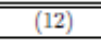 \\
\hline Log real GDP cap. & $\begin{array}{c}0.063 \\
(0.077)\end{array}$ & $\begin{array}{l}0.126^{* *} \\
(0.059)\end{array}$ & $\begin{array}{c}0.021 \\
(0.079)\end{array}$ & $\begin{array}{c}0.003 \\
(0.079)\end{array}$ & $\begin{array}{c}0.005 \\
(0.079)\end{array}$ & $\begin{array}{c}0.017 \\
(0.078)\end{array}$ & $\begin{array}{l}-1.677 \\
(1.081)\end{array}$ & $\begin{array}{c}-2.155^{* * *} \\
(0.758)\end{array}$ & $\begin{array}{l}-2.967^{*} \\
(1.722)\end{array}$ & $\begin{array}{c}-3.224^{* *} \\
(1.612)\end{array}$ & $\begin{array}{l}-3.105^{*} \\
(1.585)\end{array}$ & $\begin{array}{l}-3.187^{*} \\
(1.713)\end{array}$ \\
\hline Log real GDP cap. ${ }^{2}$ & $\begin{array}{r}-0.003 \\
(0.005)\end{array}$ & $\begin{array}{c}-0.007^{* * *} \\
(0.004)\end{array}$ & $\begin{array}{c}-0.001 \\
(0.005)\end{array}$ & $\begin{array}{l}-0.000 \\
(0.005)\end{array}$ & $\begin{array}{c}-0.000 \\
(0.005)\end{array}$ & $\begin{array}{c}-0.001 \\
(0.005)\end{array}$ & $\begin{array}{c}0.093 \\
(0.065)\end{array}$ & $\begin{array}{c}0.123^{* * *} \\
(0.045)\end{array}$ & $\begin{array}{l}0.167^{*} \\
(0.102)\end{array}$ & $\begin{array}{l}0.184^{*} \\
(0.095)\end{array}$ & $\begin{array}{l}0.178^{*} \\
(0.094)\end{array}$ & $\begin{array}{l}0.184^{*} \\
(0.101)\end{array}$ \\
\hline Log population & $\begin{array}{c}0.042^{* * * *} \\
(0.005)\end{array}$ & $\begin{array}{c}0.032^{* * * *} \\
(0.004)\end{array}$ & $\begin{array}{c}0.018^{* * * *} \\
(0.006)\end{array}$ & $\begin{array}{c}0.019^{* * * *} \\
(0.006)\end{array}$ & $\begin{array}{c}0.019^{* * * *} \\
(0.006)\end{array}$ & $\begin{array}{c}0.018^{* * * *} \\
(0.006)\end{array}$ & $\begin{array}{c}0.082^{* * * *} \\
(0.015)\end{array}$ & $\begin{array}{c}0.060^{* * *} \\
(0.015)\end{array}$ & $\begin{array}{c}-0.026 \\
(0.041)\end{array}$ & $\begin{array}{l}-0.002 \\
(0.043)\end{array}$ & $\begin{array}{l}-0.008 \\
(0.042)\end{array}$ & $\begin{array}{c}-0.019 \\
(0.041)\end{array}$ \\
\hline Market access & $\begin{array}{c}0.418^{*+*} \\
(0.066)\end{array}$ & $\begin{array}{c}0.279^{*+*}+ \\
(0.047)\end{array}$ & $\begin{array}{c}0.361^{* * *} \\
(0.068)\end{array}$ & $\begin{array}{c}0.359^{*+*} \\
(0.069)\end{array}$ & $\begin{array}{c}0.358^{*+*} \\
(0.069)\end{array}$ & $\begin{array}{c}0.369^{*+*} \\
(0.067)\end{array}$ & $\begin{array}{c}0.312 \\
(0.365)\end{array}$ & $\begin{array}{c}0.227 \\
(0.359)\end{array}$ & $\begin{array}{c}5.574^{* * *} \\
(2.115)\end{array}$ & $\begin{array}{l}3.615^{*} \\
(2.059)\end{array}$ & $\begin{array}{l}3.811^{*} \\
(2.061)\end{array}$ & $\begin{array}{l}3.984^{*} \\
(2.188)\end{array}$ \\
\hline Secondary education & $\begin{array}{c}0.002^{* * * *} \\
(0.000)\end{array}$ & $\begin{array}{c}0.001^{* * * *} \\
(0.000)\end{array}$ & $\begin{array}{c}0.002^{* * * *} \\
(0.000)\end{array}$ & $\begin{array}{c}0.002^{* * *} \\
(0.001)\end{array}$ & $\begin{array}{c}0.002^{* * *} \\
(0.001)\end{array}$ & $\begin{array}{c}0.002^{* * *} \\
(0.000)\end{array}$ & $\begin{array}{c}0.007^{* * * *} \\
(0.002)\end{array}$ & $\begin{array}{c}0.005^{* * *} \\
(0.002)\end{array}$ & $\begin{array}{c}0.000 \\
(0.003)\end{array}$ & $\begin{array}{c}0.000 \\
(0.003)\end{array}$ & $\begin{array}{l}-0.000 \\
(0.003)\end{array}$ & $\begin{array}{r}-0.002 \\
(0.003)\end{array}$ \\
\hline$\Delta$ FDI inflow ( $\%$ GDP) & $\begin{array}{c}0.012^{* *} \\
(0.005)\end{array}$ & & & & & & $\begin{array}{c}0.010 \\
(0.024)\end{array}$ & & & & & \\
\hline Globalization index & & $\begin{array}{c}0.001 \\
(0.001)\end{array}$ & & & & & & $\begin{array}{c}0.014^{* * *} \\
(0.004)\end{array}$ & & & & \\
\hline$\Delta$ FVA (\% exports) & & & $\begin{array}{l}0.080^{* *} \\
(0.038)\end{array}$ & & & & & & $\begin{array}{l}0.513^{*} \\
(0.309)\end{array}$ & & & \\
\hline$\Delta$ DVX (\% exports) & & & & $\begin{array}{c}0.059 \\
(0.073)\end{array}$ & & & & & & $\begin{array}{l}1.082^{* *} \\
(0.432)\end{array}$ & & \\
\hline$\Delta$ Term 3 (\% exports) & & & & & $\begin{array}{c}0.040 \\
(0.067)\end{array}$ & & & & & & $\begin{array}{c}1.155^{* * *} \\
(0.393)\end{array}$ & \\
\hline$\Delta$ GVC participation & & & & & & $\begin{array}{c}0.270^{*+*} \\
(0.104)\end{array}$ & & & & & & $\begin{array}{c}1.821^{* * *} \\
(0.522)\end{array}$ \\
\hline Observations & 1,529 & 2,003 & 1,129 & 1,115 & 1,115 & 1,129 & 248 & 293 & 118 & 118 & 118 & 118 \\
\hline Countries & 167 & 169 & 155 & 154 & 154 & 155 & 30 & 30 & 23 & 23 & 23 & 23 \\
\hline Accelerations included & $94 / 175$ & $108 / 175$ & $62 / 175$ & $62 / 175$ & $62 / 175$ & $62 / 175$ & $21 / 38$ & $25 / 38$ & $11 / 38$ & $11 / 38$ & $11 / 38$ & $11 / 38$ \\
\hline Year FE & Yes & Yes & Yes & Yes & Yes & Yes & Yes & Yes & Yes & Yes & Yes & Yes \\
\hline Sample & World & World & World & World & World & World & LAC & LAC & LAC & LAC & LAC & LAC \\
\hline McFadden $\mathrm{R}^{2}$ & 0.155 & 0.133 & 0.131 & 0.130 & 0.130 & 0.134 & 0.226 & 0.247 & 0.253 & 0.275 & 0.280 & 0.291 \\
\hline Pseudo $R^{2}$ & 0.129 & 0.105 & 0.105 & 0.105 & 0.105 & 0.107 & 0.200 & 0.218 & 0.231 & 0.245 & 0.249 & 0.256 \\
\hline Obeerved $\%$ of $\mathrm{EA}=1$ & 18.51 & 16.18 & 16.56 & 16.77 & 16.77 & 16.56 & 24.19 & 25.26 & 27.97 & 27.97 & 27.97 & 27.97 \\
\hline$\%$ correctly classified & 66.97 & 65.90 & 66.52 & 66.82 & 67.09 & 66.61 & 70.16 & 71.33 & 66.95 & 69.49 & 70.34 & 72.88 \\
\hline
\end{tabular}

\begin{tabular}{|c|c|c|c|c|c|c|c|c|c|c|c|c|}
\hline Panel B: Services & (1) & $(2)$ & $(3)$ & $(4)$ & $(5)$ & $(\overline{l(6)}$ & $(7)$ & $(8)$ & $(9)$ & $(10)$ & (11) & $(12)$ \\
\hline Log real GDP cap. & $\begin{array}{l}-0.146 \\
(0.101)\end{array}$ & $\begin{array}{c}-0.101 \\
(0.077)\end{array}$ & $\begin{array}{l}-0.108 \\
(0.107)\end{array}$ & $\begin{array}{l}-0.124 \\
(0.109)\end{array}$ & $\begin{array}{l}-0.128 \\
(0.109)\end{array}$ & $\begin{array}{l}-0.135 \\
(0.108)\end{array}$ & $\begin{array}{l}-0.118 \\
(0.795)\end{array}$ & $\begin{array}{l}-0.147 \\
(0.636)\end{array}$ & $\begin{array}{c}-0.664 \\
(1.242)\end{array}$ & $\begin{array}{c}-0.137 \\
(1.142)\end{array}$ & $\begin{array}{c}-0.150 \\
(1.138)\end{array}$ & $\begin{array}{l}-0.248 \\
(1.135)\end{array}$ \\
\hline Log real GDP cap. ${ }^{2}$ & $\begin{array}{c}0.008 \\
(0.006)\end{array}$ & $\begin{array}{c}0.004 \\
(0.005)\end{array}$ & $\begin{array}{c}0.006 \\
(0.006)\end{array}$ & $\begin{array}{c}0.007 \\
(0.006)\end{array}$ & $\begin{array}{c}0.007 \\
(0.006)\end{array}$ & $\begin{array}{c}0.008 \\
(0.006)\end{array}$ & $\begin{array}{l}-0.003 \\
(0.047)\end{array}$ & $\begin{array}{c}-0.001 \\
(0.037)\end{array}$ & $\begin{array}{c}0.027 \\
(0.072)\end{array}$ & $\begin{array}{l}-0.005 \\
(0.067)\end{array}$ & $\begin{array}{c}-0.004 \\
(0.066)\end{array}$ & $\begin{array}{c}0.004 \\
(0.066)\end{array}$ \\
\hline Log population & $\begin{array}{c}0.042^{*+*} \\
(0.006)\end{array}$ & $\begin{array}{c}0.028^{*+*} \\
(0.005)\end{array}$ & $\begin{array}{c}0.023^{* * *} \\
(0.008)\end{array}$ & $\begin{array}{c}0.028^{* * *} \\
(0.008)\end{array}$ & $\begin{array}{c}0.028^{* * *} \\
(0.008)\end{array}$ & $\begin{array}{c}0.026^{* *+} \\
(0.008)\end{array}$ & $\begin{array}{c}0.059^{* * *} \\
(0.015)\end{array}$ & $\begin{array}{c}0.036^{* *} \\
(0.014)\end{array}$ & $\begin{array}{c}0.040 \\
(0.034)\end{array}$ & $\begin{array}{c}0.056 \\
(0.035)\end{array}$ & $\begin{array}{c}0.057 \\
(0.035)\end{array}$ & $\begin{array}{c}0.054 \\
(0.034)\end{array}$ \\
\hline Market acoess & $\begin{array}{c}0.047 \\
(0.068)\end{array}$ & $\begin{array}{c}0.003 \\
(0.055)\end{array}$ & $\begin{array}{l}-0.014 \\
(0.074)\end{array}$ & $\begin{array}{l}-0.017 \\
(0.075)\end{array}$ & $\begin{array}{l}-0.012 \\
(0.075)\end{array}$ & $\begin{array}{c}0.012 \\
(0.074)\end{array}$ & $\begin{array}{l}-0.607 \\
(0.401)\end{array}$ & $\begin{array}{c}-0.653^{* *} \\
(0.313)\end{array}$ & $\begin{array}{c}-2.120^{* *} \\
(0.986)\end{array}$ & $\begin{array}{l}-2.121^{*} \\
(1.114)\end{array}$ & $\begin{array}{l}-2.165^{\circ} \\
(1.133)\end{array}$ & $\begin{array}{c}-2.424^{* *} \\
(1.121)\end{array}$ \\
\hline Secondary education & $\begin{array}{c}0.002 * * * \\
(0.001)\end{array}$ & $\begin{array}{c}0.001 \\
(0.001)\end{array}$ & $\begin{array}{l}0.0002^{* *} \\
(0.001)\end{array}$ & $\begin{array}{c}0.001^{* *} \\
(0.001)\end{array}$ & $\begin{array}{c}0.001^{* *} \\
(0.001)\end{array}$ & $\begin{array}{l}0.002^{* *} \\
(0.001)\end{array}$ & $\begin{array}{c}0.001 \\
(0.002)\end{array}$ & $\begin{array}{c}0.000 \\
(0.002)\end{array}$ & $\begin{array}{c}-0.001 \\
(0.003)\end{array}$ & $\begin{array}{c}0.001 \\
(0.003)\end{array}$ & $\begin{array}{c}0.001 \\
(0.003)\end{array}$ & $\begin{array}{c}-0.002 \\
(0.003)\end{array}$ \\
\hline$\Delta$ FDI inflow (\% GDP) & $\begin{array}{l}-0.012^{*} \\
(0.008)\end{array}$ & & & & & & $\begin{array}{l}0.049^{*} \\
(0.028)\end{array}$ & & & & & \\
\hline Globalization index & & $\begin{array}{c}0.003^{* * *} \\
(0.001)\end{array}$ & & & & & & $\begin{array}{c}0.009^{* * * *} \\
(0.003)\end{array}$ & & & & \\
\hline$\Delta$ FVA (\% exports) & & & $\begin{array}{c}0.234^{* * *} \\
(0.063)\end{array}$ & & & & & & $\begin{array}{c}1.048^{* * * *} \\
(0.339)\end{array}$ & & & \\
\hline$\Delta$ DVX (\% exports) & & & & $\begin{array}{c}-0.230^{* *} \\
(0.092)\end{array}$ & & & & & & $\begin{array}{c}-0.040 \\
(0.344)\end{array}$ & & \\
\hline$\Delta$ Term 3 (\% exports) & & & & & $\begin{array}{c}-0.214^{* *} \\
(0.091)\end{array}$ & & & & & & $\begin{array}{c}0.037 \\
(0.351)\end{array}$ & \\
\hline$\Delta$ GVC participation & & & & & & $\begin{array}{c}0.377^{* *} \\
(0.161)\end{array}$ & & & & & & $\begin{array}{c}1.439 * * \\
(0.543)\end{array}$ \\
\hline Observations & 1,250 & 1,621 & 1,025 & 1,016 & 1,016 & 1,025 & 198 & 250 & 124 & 124 & 124 & 124 \\
\hline Countries & 167 & 168 & 153 & 152 & 152 & 153 & 28 & 29 & 24 & 24 & 24 & 24 \\
\hline Accelerations included & $84 / 162$ & $96 / 162$ & $80 / 162$ & $79 / 162$ & $79 / 162$ & $80 / 162$ & $12 / 26$ & $15 / 26$ & $11 / 26$ & $11 / 26$ & $11 / 26$ & $11 / 26$ \\
\hline Year FE & Yes & Yes & Yes & Yes & Yes & Yes & Yes & Yes & Yes & Yes & Yes & Yes \\
\hline Sample & World & World & World & World & World & World & LAC & $\mathrm{LAC}$ & LAC & LAC & LAC & LAC \\
\hline McFadden $\mathrm{R}^{2}$ & 0.165 & 0.161 & 0.162 & 0.154 & 0.153 & 0.155 & 0.221 & 0.232 & 0.250 & 0.169 & 0.169 & 0.217 \\
\hline Pseudo $\mathrm{R}^{2}$ & 0.145 & 0.133 & 0.151 & 0.143 & 0.143 & 0.145 & 0.176 & 0.178 & 0.219 & 0.160 & 0.160 & 0.196 \\
\hline Observed $\%$ of $\mathrm{EA}=1$ & 20.80 & 18.26 & 23.61 & 23.52 & 23.52 & 23.61 & 18.69 & 17.60 & 25.00 & 25.00 & 25.00 & 25.00 \\
\hline$\%$ correctly classified & 69.68 & 69.34 & 68.49 & 66.63 & 67.03 & 67.71 & 67.68 & 67.60 & 71.77 & 63.71 & 63.71 & 71.77 \\
\hline
\end{tabular}

Notes: Probit estimates. The dependent variable is a dummy for the timing of goods (Panel A) and services (Panel B) export acoelerations (EA) which equals 1 over the 3-year window centered on the initiation date. Coefficients are marginal probabilities evaluated at the sample means. Robust standard errors are given in parenthesis. ${ }^{*},{ }^{* *}$ and ${ }^{* * *}$ denote statistical significanoe at the $10 \%, 5 \%$ and $1 \%$ level respectively. All covariates are lagged by 2 years. $\Delta$ indicates 3-year changes. LAC refers to Latin America and the Caribbean. The description and souroe of variables are provided in Table A3. 


\section{Robustness and Sensitivity Checks}

36. In this section, we test the robustness of our results to the choice of parameters in the identification of export accelerations and to the method of estimation. We also examine whether our results remain unchanged when using more disaggregated export data, splitting the period of study, applying an income and geographical sample breakdown, and accounting for oil-dependence. Finally, we consider the symmetric case of export decelerations.

\section{Choice of Parameters}

\section{The first set of robustness checks relates to the parameters used in the}

identification of an export acceleration. The baseline model defined an export acceleration as a significant increase in export growth that is sustained for at least 7 years. We test whether shortening the horizon to 5 years or lengthening it to 9 years makes any difference (Table A4). ${ }^{25}$ Results with the 5-year horizon confirm the baseline estimates, with even larger and more significant coefficients on exchange rate, diversification and tariff-related variables. Capital account openness is now positively associated with services export accelerations, while banking sector reforms appear to undermine goods export takeoffs. This is corroborated with findings from the 9-year horizon where both domestic and international financial liberalization are negatively associated with the timing of goods export accelerations. ${ }^{26}$ The main findings are also robust to tightening or relaxing the parameters of criterion 2. First, we modify the condition that real average export growth during takeoff increases by one third from the baseline growth rate by alternately setting the threshold to one tenth (1.1) and two (Table A5). Second, we modify the change in export growth requirement by successively lowering the acceleration threshold to 2 percentage points and raising it to 4 percentage points (Table A6). Our results are hardly affected by the varying cutoffs.

\section{Alternative Estimation Methods}

38. We also estimate a logit model, where $\phi$ in the baseline specification becomes the cumulative logistic distribution instead of the cumulative normal distribution. Both probit and logit models usually yield identical results, but divergences may arise as a result of very unbalanced samples with fewer ones than zeros (Carrère and de Melo, 2012). Despite the unbalanced nature of our sample, logit estimates remain remarkably similar to the

\footnotetext{
25 The 5-year horizon yields 276 and 240 surges in goods and services exports respectively against 111 and 105 only using the 9-year horizon. Corresponding figures for LAC stand at 50 goods and 42 services accelerations with the 5-year horizon versus 26 and 19 identified with the 9-year horizon.

${ }^{26}$ It is worth noting that since the 9-year horizon is more data demanding, it drastically reduces the number of observations, yielding non-significant results in the case of tariff or GVC-related variables that are available over a limited time span to begin with. A curious result is that diversification at the extensive margin is now negatively associated with goods export accelerations. Likewise, the KOF index of globalization now bears the wrong sign.
} 
previous results (Table A7). In the same vein, we fit a logit model corrected for rare occurrence bias as suggested by King and Zeng (2001). This estimation method addresses the shortcoming of the standard logit and probit regressions which can underestimate the probability of occurrence of an export acceleration given the overwhelming prevalence of observations for which $E A_{i t}=0$. As displayed in Table A7, tariffs on imported manufactures and agricultural reforms are no longer statistically associated with goods export takeoffs. Likewise, diversification at the extensive margin fails to predict goods accelerations in LAC, while FDI inflows no longer enter with a positive coefficient. However, the bulk of our results remain unchanged. Some coefficients even gain in statistical significance: this is the case of human capital which has now some bearing on the timing of export accelerations in LAC. Both domestic and international financial liberalization now appear to strongly and negatively correlate with goods export takeoffs while the sign of the association turns positive in the case of services.

\section{Disaggregating Exports}

\section{We run additional robustness checks by distinguishing between manufactures} and non-fuel primary commodities on the one hand; and traditional and modern services on the other. ${ }^{27}$ This complements the earlier analysis on aggregate goods and services exports. Table A8 shows that infrastructure quality, real effective depreciation, agricultural reforms and FDI matter for export takeoffs in both manufactures and primary products, consistent with baseline results. Coefficients on investment, tariffs on imported goods, globalization and growing participation in longer value chains through forward linkages become statistically significant when goods are limited to primary products. Other correlates including human capital and downstream involvement in GVCs turn out to matter for manufactures only, reflecting their skill-intensive and sophisticated nature, and the fact that their production and export may hinge on the import of raw materials from upstream countries in GVCs. Table A8 also reveals that the previously uncovered negative association between the liberalization of networks industries and goods export accelerations is driven by manufactures.

\section{Both traditional and modern commercial services strongly correlate with human capital and governance indicators, consistent with previous findings. However, neither financial liberalization nor increased competition in the telecommunication and electricity markets seem to precede either type of services export acceleration. Overall, export surges in modern services seem to drive most of the results at the aggregate level, while the negative association between FDI inflows and services export accelerations (Table 4) is attributable to traditional services. In the same vein, macroeconomic uncertainty positively correlates with}

\footnotetext{
${ }^{27}$ This exercise is carried out for the world sample only. Manufactures exports are obtained by aggregating SITC rev. 2 sections 5 to 8 , excluding division 68. Non-fuel primary commodities refer to food (sections $0,1,4$ and division 22) and agricultural raw materials (section 2 excluding divisions 22, 27 and 28). Traditional services include transportation (BOP code 205) and travel (BOP code 236), while remaining commercial services are aggregated under modern services.
} 
surges in traditional services, and negatively with export accelerations in modern services, explaining the non-significant coefficient on REER volatility found at the aggregate level in Table 1.

\section{Splitting the Period of Study}

41. We test the robustness of the regressions before and after 2000 (Table A8). The split is driven by Figures 1 and 2, which highlighted a concentration of export accelerations in the second half of the 1980s and the first half of the 2000s. Human capital, macroeconomic uncertainty, currency depreciation and GVC participation, especially through backward linkages, ${ }^{28}$ remain associated with goods export accelerations over both sub-samples. However, other correlates vary depending on the period considered. For instance, surges which took place during the 1980s and 1990s were on average preceded by diversification at the intensive margin, ${ }^{29}$ agricultural reforms and FDI inflows; while increased capital account openness reduced their probability of occurrence. In contrast, post2000 initiations were primarily triggered by domestic enabling factors such as investment growth and government quality. ${ }^{30}$ Diversification at the extensive margin and lower tariffs also helped, ${ }^{31}$ while banking sector reforms and the liberalization of the telecommunication and electricity markets hindered the emergence of goods export accelerations.

\section{Irrespective of the time period considered, services export takeoffs were} preceded by improved government quality, human capital, diversification, as well as growth in FVA and network industries liberalization. ${ }^{32}$ Democracy, real effective depreciation, diversification at the extensive margin, and agricultural reforms were conducive to services surges in the 1980s-1990s. Investment and diversification at the intensive margin were key in triggering accelerations in the post-2000 period. Financial liberalization in the form of domestic banking sector reforms and increased capital account openness also contributed to boosting high and sustained services export growth over the period.

\section{Income and regional disparities}

43. As an additional robustness check, we investigate whether macroeconomic conditions associate with export takeoffs are the same regardless of the level of

\footnotetext{
${ }^{28}$ Coefficients on GVC participation and FVA content of exports are statistically significant at the 11 and 13 percent levels respectively.

${ }^{29}$ This also went hand in hand with concentration at the extensive margin.

${ }^{30}$ Transition towards democracy bears the right sign before 2000, but correlates negatively with goods export accelerations in the 2000 s.

${ }^{31}$ However, tariffs enter with a statistically significant sign over the post-2000 sub-sample only, probably due to limited data availability before 2000 .

32 Macroeconomic uncertainty enters with the right sign during the 1980s-1990s, but correlates positively with export takeoffs after 2000 .
} 
development. Results for advanced economies (AEs) and emerging and developing market countries (EMs) are summarized in Table A9. They indicate that the bulk of our baseline findings is driven by EMs. In particular, developing countries will benefit most from enhanced human capital, government quality, agricultural reforms, globalization and GVC participation. However, further growth in investment and FDI inflows in AEs is negatively associated with accelerations in goods and services exports respectively, possibly reflecting high-income countries' closeness to the technology frontier. The liberalization of telecommunication and electricity markets is not negatively associated with goods export accelerations anymore, while it remains positively correlated with services surges irrespective of the sub-sample considered (albeit at the 13 percent significance level in the case of EMs).

\section{Some correlates also enter with different signs depending on the sub-sample}

considered. These results shed light on the non-significant or peculiar results obtained with the entire sample: i) export accelerations are more likely to occur in a context of progress toward democracy in EMs, while the inverse holds in AEs; ii) a depreciated exchange rate promotes surges in EMs while currency appreciation seems to matter for AEs; iii) export diversification has some bearing on the initiation of accelerations in EMs, contrary to AEs which tend to enjoy high and sustained export growth as a result of concentration, consistent with Cadot et al. (2011). ${ }^{33}$

45. We also look into commonalities and differences across EMs. Table A9 displays results for emerging Asia (EMA), Sub-Saharan Africa (SSA) and MENA. ${ }^{34}$ Both LAC and SSA share human capital accumulation and GVC participation as important pre-conditions in fostering goods export takeoffs ${ }^{35}$; but contrary to LAC, the role of cheaper imported goods and network industries liberalization is more straightforward in SSA. Progress toward democracy also appears as a stringent requirement that ought to meet SSA to raise its likelihood of experiencing strong and sustained services export accelerations. Diversification matters in LAC and MENA which both host commodity-dependent countries, but the two regions seem to diverge in many respects. For instance, capital account openness promotes the emergence of goods export surges in LAC, while hindering them in MENA. Similarly, real exchange rate appreciation and growth in foreign value-added content of exports negatively correlate with accelerations in MENA. Disparities are also noticeable when contrasting LAC and EMA: agricultural reforms enter with a negative sign in EMA. Similar to AEs, transition toward democracy and currency depreciation are negatively associated

\footnotetext{
${ }^{33}$ Cadot et al. (2011) evidence a hump-shaped relationship between diversification and GDP per capita: diversification occurs at the early stages of development, followed by concentration at high-income levels. They find that this pattern mostly takes place along the extensive margin of trade.

${ }^{34}$ MENA includes Afghanistan and Pakistan. We do not provide results for emerging Europe and CIS due to insufficient number of observations and export accelerations included.

${ }^{35}$ In SSA, benefits of GVC participation seem to accrue from forward linkages only.
} 
with goods export accelerations in the region, while policies aimed at encouraging the development of bond and equity markets, as well as easing access of the domestic stock market to foreigners promote accelerations in both goods and services. In contrast, domestic financial liberalization through banking sector reforms and capital account openness make surges less likely to occur in EMA. Nonetheless, some domestic enabling factors such as investment growth and government quality matter for launching services export accelerations in both LAC and EMA.

\section{Oil Dependence}

46. We also test whether our results are sensitive to oil-dependence. Specifically, we identify the sub-sample of EMs whose main source of export earnings is fuel (Table A10). ${ }^{36}$ We find that the timing of goods export accelerations in these countries is particularly sensitive to the availability of cheaper imported goods, possibly because import-competing domestic industries have not flourished due to oil-dependence and lack of economic diversification. In this respect, fuel-rich countries were able to experience goods accelerations by exporting a more balanced mix of existing products, despite a concentrated export basket at the extensive margin, thus yielding a non-significant coefficient on the aggregate Theil index. The prevalence of the oil sector may also explain why GVC participation enters negatively, contrary to baseline estimates. On the other hand, infrastructure and government quality appear as important pre-conditions for fuel-rich countries to reap the benefits of high and sustained export growth, probably because they are more prone to rent capture. Another remarkable result is the positive association between currency appreciation and services export accelerations in oil-dependent countries, while this relationship is negative for goods exports, consistent with the Dutch disease effect.

\section{Export Decelerations}

\section{As a final robustness check, we focus on instances of severe export collapses to investigate whether correlates of export takeoffs also play a role in explaining instances of significant fall in export growth. For this purpose, we symmetrically define an export deceleration as an episode of drastic reduction in export growth that is sustained for at least seven years. ${ }^{37}$ The filters yield 21 and 12 decelerations in goods and services exports respectively, among which 9 and 3 took place in LAC. As an export collapse turns out to be}

\footnotetext{
${ }^{36}$ Recall that accelerations are identified based on aggregate export data excluding fuels and ores and metals. Surges uncovered for oil-rich countries somehow reflect their ability to experience high and sustained goods export growth despite their oil-dependence.

${ }^{37}$ Specifically, we require that i) real average export growth during the seven-year period immediately following the collapse date is negative; ii) it decreases by one third from the baseline growth rate and is at least 3 percentage points below it; iii) the maximum level of exports observed during the collapse period is lower than the minimum level of exports observed during the baseline years; iv) the real average export growth rate during the collapse period, excluding the year of weakest growth, is lower than real average growth during baseline.
} 
much rarer than a surge, Table A10 presents results obtained by using King and Zeng (2001)'s logit estimator corrected for rare occurrence bias. We find that on average, goods export decelerations are preceded by macroeconomic uncertainty. Although investment growth and transition toward democracy did not spur export surges (Table 1), they now appear to reduce the likelihood of decelerations. Similarly, in LAC infrastructure quality matters for raising the odds against export collapses. The positive association between REER volatility and services export decelerations confirms the peculiar results obtained in previous robustness checks. ${ }^{38}$

\section{Symmetric to the baseline results, currency appreciation and export} concentration raise the probability of observing an export deceleration. ${ }^{39}$ Again, the negative association between the Theil index and the dummy for the timing of services export deceleration is consistent with previous robustness checks, and may be ascribed to AEs. In addition, tariffs enter positively, further lending credence to the main results, while trade openness negatively correlates with export collapses. Interestingly, relaxing restrictions on capital transactions appears to be a hedge against decelerations, just like bond and equity market liberalization in LAC; possibly by alleviating firms' financing constraints. Along the same lines, further FDI inflows, and more broadly globalization, make the occurrence of decelerations less likely. The relationship between GVC participation and export collapses is however less straightforward: downstream involvement in multi-stage trade process appears to negatively correlate with the dependent dummy variable consistent with the counterpart result for accelerations. Conversely, upstream involvement through forward linkages is positively associated with decelerations.

\section{Post-Surge Performance}

\section{A. Synthetic Control Method}

\section{Do countries that experience export accelerations enjoy higher GDP per capita} and lower unemployment and income inequality during the period following the surge? To address this question, we resort to the synthetic control method (SCM), a transparent statistical methodology developed by Abadie and Gardeazabal (2003) and extended by Abadie et al. (2010). ${ }^{40}$ Formally, SCM compares a treated country with an estimated counterfactual, the so-called synthetic control, which is a linear combination of untreated

\footnotetext{
${ }^{38}$ Based on previous robustness checks, this result may be driven by a combination of i) collapses in traditional commercial services exports; or ii) decelerations that occurred after 2000, possibly in iii) AEs or EMAs.

${ }^{39}$ The coefficient on the REER index is statistically significant at the 13 percent level for the LAC sample.

${ }^{40}$ Since its introduction, SCM has been applied in various studies. For instance, Billmeier and Nannicini (2013) use the methodology to quantify the impact of economic liberalization on real GDP per capita, while Abadie et al. (2015) assess the economic impact of the 1990 German reunification on West Germany. Hannan (2016) analyses the impact of trade agreements on exports, whereas Adhikari et al. (2016) examine the economic implications of reforms in selected industrialized economies.
} 
countries. Weights are chosen so that the synthetic control resembles the treated country in all relevant pre-treatment characteristics which may include pre-treatment realizations of the outcome variable.

Consider a sample of $j=1, \ldots, J+1$ countries observed over time $t=1, \ldots, T$ among which country $j=1$ is the treated unit ("surge country") while the other countries constitute the "donor pool", i.e. the set of potential control units ("non-surge countries"). In our context, the initiation year of the export acceleration is identified as the treatment date. Let $T_{0}$ be the number of pre-intervention periods, with $1 \leq T_{0} \leq T$. In addition, let $Y_{i t}^{S}$ be the outcome of the surge country, and $Y_{i t}^{N S}$ the outcome of any country in the absence of an export acceleration. Subsequently, the observed outcome is given by:

$$
Y_{i t}=Y_{i t}^{N S}+\alpha_{i t} D_{i t}
$$

where $\alpha_{i t}=Y_{i t}^{S}-Y_{i t}^{N S}$ is the effect of the occurrence of the export acceleration for country $i$ at time $t$, and $D_{i t}$ a dummy variable. Given that only the first country experiences an acceleration and only after $T_{0}$ :

$$
D_{i t}=\left\{\begin{array}{lr}
1 \text { if } i=1 \text { and } t>T_{0} \\
0 \quad \text { otherwise }
\end{array}\right.
$$

Subsequently, we aim to estimate $\left(\alpha_{1 T_{0+1}}, \ldots, \alpha_{1 T}\right)$, i.e. the dynamic treatment effect for each year following the initiation date of the export acceleration, knowing that:

$$
\alpha_{i t}=Y_{i t}^{S}-Y_{i t}^{N S}=Y_{1 t}-Y_{1 t}^{N S} \text { for } t>T_{0}
$$

Since $Y_{1 t}^{N S}$ is not observed for the surge country over the post-acceleration period $\left[T_{0}+1, \ldots, T\right]$, SCM constructs a synthetic control group that yields a reasonable estimate for this missing potential outcome. Following Abadie et al. (2010), $Y_{i t}^{N S}$ is given by a factor model:

$$
Y_{i t}^{N S}=\delta_{t}+\theta_{t} Z_{i}+\lambda_{t} \mu_{i}+\epsilon_{i t}
$$

where $\delta_{t}$ is an unknown common factor with constant factor loadings across countries, $Z_{i}$ is a vector of observed covariates with coefficients $\theta_{t}, \lambda_{t}$ is a vector of unobserved common factors, $\mu_{i}$ is a vector of unknown factor loadings, and $\epsilon_{i t}$ are error terms. Unlike in standard difference-in-differences models, this specification allows controlling for the effect of timevarying unobserved heterogeneity $\left(\lambda_{t} \mu_{i}\right)$.

Let $W=\left(w_{2}, \ldots, w_{J+1}\right)$ be a vector of weights such that $w_{j} \geq 0$ for $j=2, \ldots, J+1$ and $w_{2}+$ $\cdots+w_{J+1}=1$. Each $W$ then represents a potential synthetic control, i.e. one particular weighted average of control units. Accordingly, the outcome variable for each potential synthetic control unit is given by: 


$$
\sum_{j=2}^{J+1} w_{j} Y_{j t}=\delta_{t}+\theta_{t} \sum_{j=2}^{J+1} w_{j} Z_{j}+\lambda_{t} \sum_{j=2}^{J+1} w_{j} \mu_{j}+\sum_{j=2}^{J+1} w_{j} \epsilon_{j t}
$$

Suppose that there is an optimal vector $W^{*}=\left(w_{2}^{*}, \ldots, w_{J+1}^{*}\right)$ such that:

$$
\sum_{j=2}^{J+1} w_{j}^{*} Y_{j 1}=Y_{11} ; \sum_{j=2}^{J+1} w_{j}^{*} Y_{j 2}=Y_{12}, \ldots, \sum_{j=2}^{J+1} w_{j}^{*} Y_{j T_{0}}=Y_{1 T_{0}} \text { and } \sum_{j=2}^{J+1} w_{j}^{*} Z_{j}=Z_{1}
$$

Then equation (6) provides an estimator for $\alpha_{i t}$ in periods $T_{0}+1, \ldots, T$.

$$
\widehat{\alpha_{\imath t}}=Y_{1 t}-\sum_{j=2}^{J+1} w_{j} Y_{j t} \text { for } t>T_{0}
$$

In practice, $W^{*}$ is selected such that equation (5) holds approximately. Specifically, let $X_{1}$ be the vector of pre-surge characteristics for the treated country, and $X_{0}$ the matrix containing the same variables for the units in the donor pool. ${ }^{41} W^{*}$ is then chosen to minimize the distance $\left\|X_{1}-X_{0} W\right\|$.

In other words, the synthetic control algorithm estimates the missing counterfactual as a weighted average of the outcomes of potential controls. The weights are chosen so that the presurge outcome and the covariates of the synthetic control are, on average, very close to those of the surge country. The quality of the pre-surge fit reached by the SCM algorithm is gauged using the root mean squared prediction error (RMSPE) which measures the lack of fit between the path of the outcome variable for the surge country and its synthetic counterpart before the export acceleration date:

$$
R M S P E=\sqrt{\frac{1}{T_{0}} \sum_{t=1}^{T_{0}}\left(Y_{i t}-\sum_{j=2}^{J+1} w_{j}^{*} Y_{j t}\right)^{2}}
$$

Furthermore, statistical inference is derived through "in-space placebo studies" following Abadie et al. (2010). Formally, we iteratively reassign the export acceleration date to every potential control, shifting the true surge country to the donor pool, and estimate the associated dynamic treatment effects over $\left[T_{0}+1, \ldots, T\right]$. Hence, in each iteration, we proceed as if the control country in the donor pool experienced an export acceleration in the same year as the "true" surge country. The rationale behind these falsification tests is to assess whether the estimated effect of the export acceleration could be driven entirely by chance. If the exercise yields an unusually large treatment effect for the surge country relative to the placebo treatment

\footnotetext{
${ }^{41}$ The pre-surge characteristics in $X_{1}$ and $X_{0}$ may include pre-surge values of the outcome variable.
} 
effects, this would be suggestive of a statistically significant effect of the export acceleration for the surge country.

\section{B. Data and Case Study Selection}

50. In our context, we use SCM to implement two data-driven country-case studies focused on Brazil and Peru. The former experienced a goods export acceleration in 2000 while the latter witnessed a surge in services exports in 2005 (Table A2). For each country, we compare the post-acceleration trajectory of real GDP per capita, unemployment, and income inequality with the trajectory of a combination of similar but untreated economies. The estimated dynamic treatment effect of the export acceleration is given by the difference in the post-surge values of each outcome variable between the treated country and its synthetic control. ${ }^{42}$

51. To implement SCM, we calibrate the synthetic control over the five pre-surge years immediately preceding the export acceleration date, and restrict the sample period to seven post-surge years. ${ }^{43} \mathrm{We}$ also exploit the flexibility of the methodology to maximize the quality of the counterfactual constructed by the algorithm. First, we exclude from the donor pool countries that also experienced an export takeoff sometime over the thirteen-year sample window. This mitigates concerns over how well the synthetic control is reproducing the outcome that would have been observed for the surge country in the absence of the export acceleration. Second, we restrict the sample of potential control units to emerging market and developing countries as identified by the IMF. ${ }^{44}$ By solely retaining control countries that belong to the same income group as the surge country, we aim to increase the "common support" shared by the treated unit and its synthetic counterpart (e.g. cultural and geographic proximity). ${ }^{45}$ As a robustness check, we later relax this condition and expand the set of control units to all available countries regardless of the income level.

\section{For each outcome of interest, we choose a vector of covariates for which we} require the treated unit and its synthetic counterpart to exhibit similar pre-acceleration values (equation 5). The vector of covariates associated with the first outcome of interest, real GDP per capita, comprises a set of standard growth determinants, namely population growth, investment as a share of GDP, government quality and human capital (Barro, 1991).

\footnotetext{
${ }^{42}$ We initially implemented SCM for all LAC countries with available data for the outcome variable. However, poor pre-treatment fit quality and data restrictions led us to only focus on two illustrative case studies, for which the pre-treatment fit was of reasonable quality for all three outcome variables of interest.

${ }^{43}$ This choice is largely dictated by the availability of unemployment and income inequality series.

${ }^{44}$ World Economic Outlook Database, October 2015.

${ }^{45}$ We also implemented SCM with a donor pool exclusively consisting of LAC countries but were faced with a trade-off: despite reducing cross-country heterogeneity, this geographical restriction resulted in a drastic shrinking of the sample size, yielding a rather poor pre-surge fit.\}
} 
The latter is captured by the Human Assets Index (HAI) from FERDI which combines both education and health dimensions of human capital. We also include a crisis dummy from Laeven and Valencia (2008, 2012\} to account for possible post-surge output effects of a banking, currency and/or sovereign debt crisis that may have occurred before the acceleration date (Cerra and Saxena, 2008). Population dynamics and human capital are also accounted for in the vector of covariates pertaining to unemployment, along with the level of development, inflation and the share of urban population.

53. We also choose a vector of covariates for income inequality, captured by the Gini index. This set includes i) GDP per capita, to account for the non-linear relationship between inequality and the level of development (Kuznets, 1955; Barro, 2000); ii) government spending as a share of GDP taken as a proxy for redistributive policies (Perotti, 1992; DablaNorris et al., 2015); iii) the ratio of female to male labor force participation rate, a key aspect of gender inequality which is strongly associated with income inequality (Gonzales et al., 2015); iv) the HAI to capture the skill premium in line with Mincer (1958) and the quality of human capital in general; and v) population growth. Furthermore, we also include the outcome variable measured at each of the five years before the export acceleration in order to maximize the goodness of fit. ${ }^{46}$ Table $\mathrm{A} 3$ in the appendix provides the definition and source of the variables.

\section{Results}

\section{Figure 10 contrasts the evolution of the level of real GDP per capita,} unemployment and income inequality in Brazil and Peru with that of their synthetic control. The extent to which the solid red line (treated unit) and the dashed blue line (synthetic control unit) coincide before the export acceleration date reflects the quality of the pre-treatment fit reached by the SCM algorithm. Conversely, any divergence observed after the initiation year captures the dynamic treatment effect of the export takeoff. Table 6 provides the list of countries from the donor pool used in the construction of each synthetic control, along with their associated weights. The means of the covariates and outcomes are displayed in Table 7. In addition, as explained earlier, the validity of the SCM results is tested through placebo experiments. For each outcome of interest, the solid red line in Figure 11 presents the difference between the treated country and its synthetic control, while the dotted grey lines depict the difference between each of the treated country's potential controls and their respective synthetic control. ${ }^{47}$

\footnotetext{
${ }^{46}$ For each outcome of interest, countries with missing data over the 13-year window are excluded.

47 Following Abadie et al. (2010, 2011), placebo studies based on countries with poor fit do not provide information to measure the relative rarity of the post-surge gap obtained for the treated country. Consequently, we discard countries whose pre-surge RMSPE is larger than the "true" treated country's RMSPE by more than the sample median.
} 
55. Brazil's post-acceleration real GDP per capita trajectory appears to outperform its synthetic counterpart's (Figure 10 (a)), a convex combination of Venezuela, Mexico, Jamaica and Malaysia (Table 6). However, this result does not survive placebo testing as 7 out of the 12 permutations are above the baseline effect uncovered for Brazil. ${ }^{48}$ Despite the lack of a robust effect on output, Figure 10 (c) shows that Brazil's unemployment rate was almost 14 percent lower than the counterfactual by three years after the takeoff, and 22 percent lower by seven years later. Although several fake experiments show stronger results than the baseline in the immediate years following the surge, the associated gaps in unemployment start reducing after $T_{0}+3$ (Figure $11(\mathrm{c})$ ). This lends some credence to the quality of the baseline effect. Brazil also enjoyed a steeper reduction in income inequality after 2000, with a Gini index that stood at 55.23 in 2007, against 58.28 for the synthetic control constructed as an average of, Botswana, South Africa, El Salvador and Nigeria (Figure 10 (e) and Table 6). The placebo test in Figure 11 (e) confirms the robustness of this result, as only 2 out of 11 permutations fare better than the treated unit

56. On the other hand, results suggest that Peru was better able to reap the benefits of the services export acceleration it experienced in 2005. Peru and its synthetic control started at comparable levels of GDP per capita before the surge, but Peru's GDP per capita was almost 14 percent higher than the estimated counterfactual three years after the acceleration, and 22 percent larger seven years later (Figure 10 (b)). The placebo test in Figure 11 (b) confirms the robustness of this result as only one out of the 16 fake experiments yields a consistently larger gap in GDP per capita than the one uncovered for Peru. Job creation seems to be an important channel through which the export takeoff positively affected output. Peru's unemployment rate was 22 percent lower than its synthetic control's at $T_{0}+3$-- a convex combination of Burkina Faso, Fiji, Kazakhstan, Yemen and Venezuela (Table 6) -- and almost 38 percent lower seven years later (Figure 10 (d)). In addition, Figure 10 (f) shows that Peru's Gini index dropped sharply relative to its synthetic counterpart's and was 8 percent lower seven years after the surge. Reduced income inequality seems to have contributed to Peru's higher GDP per capita after the 2005 services export surge. The placebo tests lend strong support to these conclusions as none of the other 21 permutations performed in the case of unemployment yields a line that is consistently lower than Peru's over the post-acceleration period (Figure 11 (d)). As for income inequality, only 1 out of 7 placebo exercises outperforms the treated unit (Figure 11 (f)).

\footnotetext{
${ }^{48}$ When enlarging the pool of potential controls to all available untreated countries, Brazil's post-acceleration real GDP per capita appears to be larger than its synthetic control's, although with decreasing dynamic treatment effects. Yet again, this result does not survive the validity checks performed through falsification tests.
} 
Figure 10. Post-Acceleration Performance in Brazil and Peru

(a) Real GDP per capita, Brazil

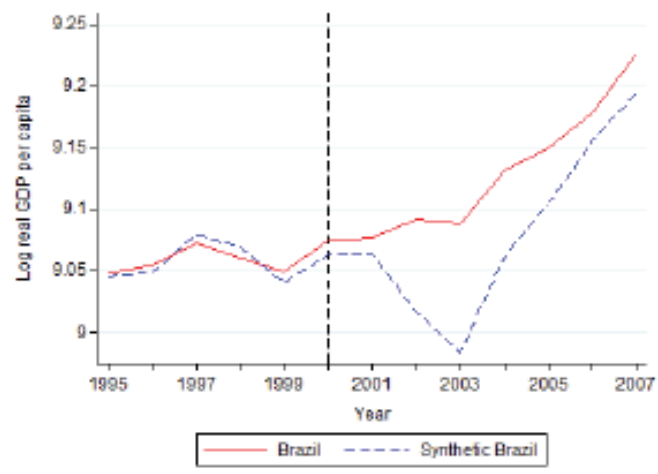

(c) Unemployment, Brazil

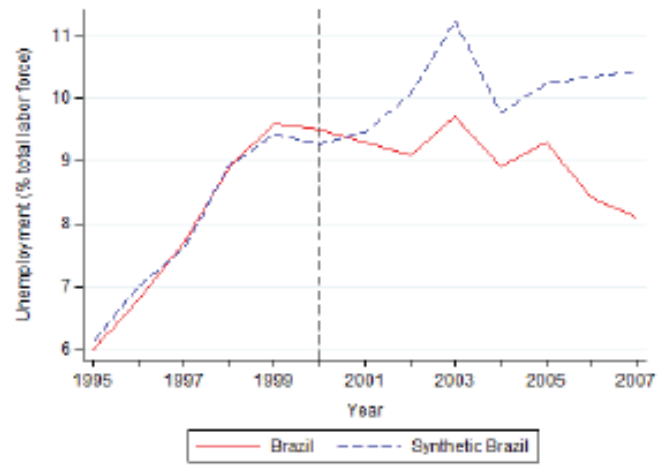

(e) Gini index, Brazil

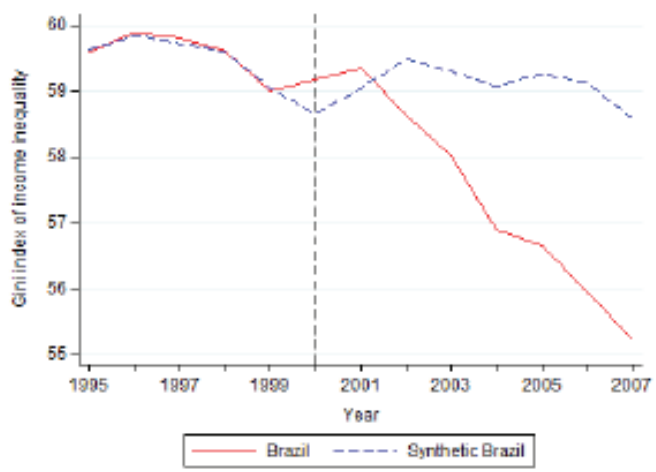

(b) Real GDP per capita, Peru

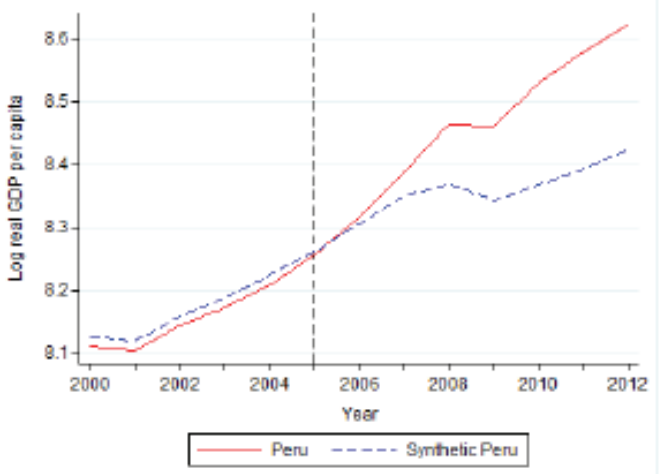

(d) Unemployment, Peru

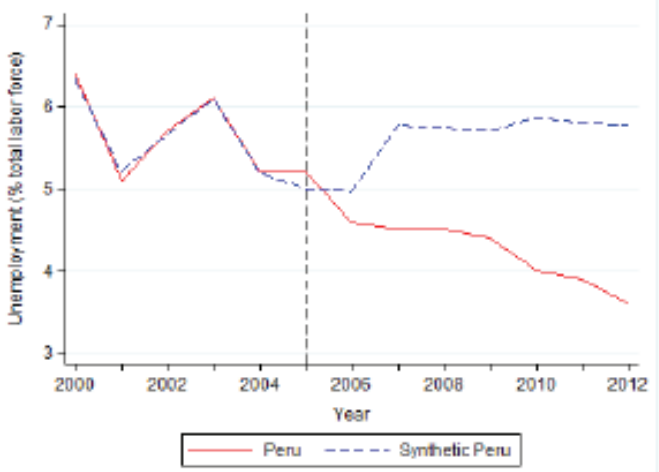

(f) Gini index, Peru

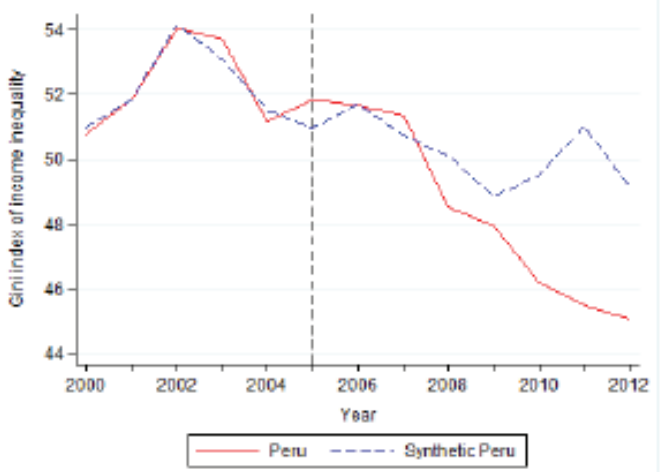

Notes: Authors' calculations based on WDI, FERDI, ICRG and Laeven and Valencia (2008, 2012). The solid red line and the dashed blue line represent the time series of the outcome variable of interest for the treated unit and its synthetic counterpart respectively. The donor pool from which the synthetic control is constructed is restricted to the sample of emerging market and developing economies as defined by the IMF World Economic Outlook Database (October 2015). It further excludes countries with missing data for the outcome variable over the 5 years preceding the surge date and 7 years following it. The description and source of variables are provided in Table A3. 
Figure 11. Placebo Experiments

(a) Real GDP per capita, Brazil

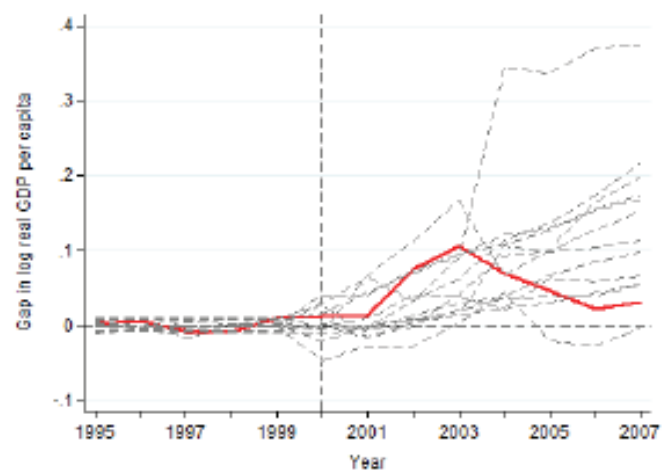

(c) Unemployment, Brazil

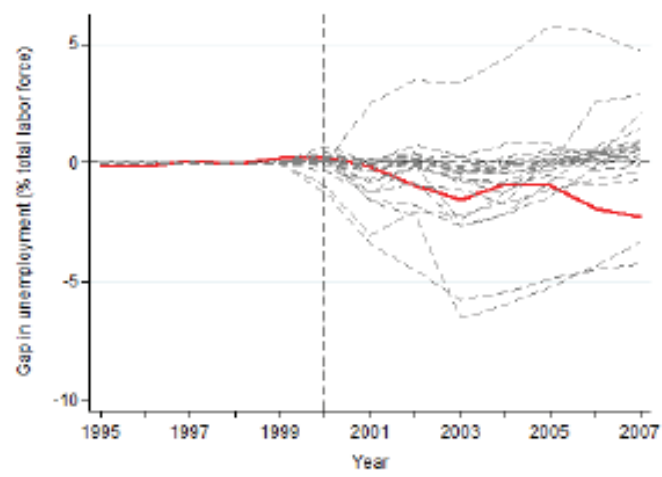

(e) Gini index, Brazil

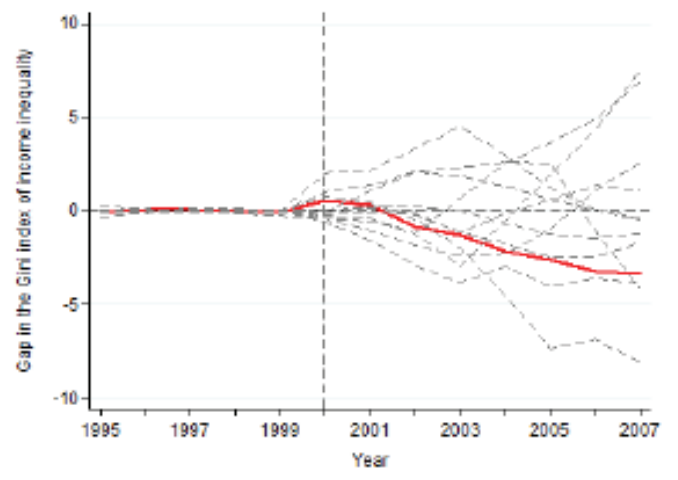

(b) Real GDP per capita, Peru

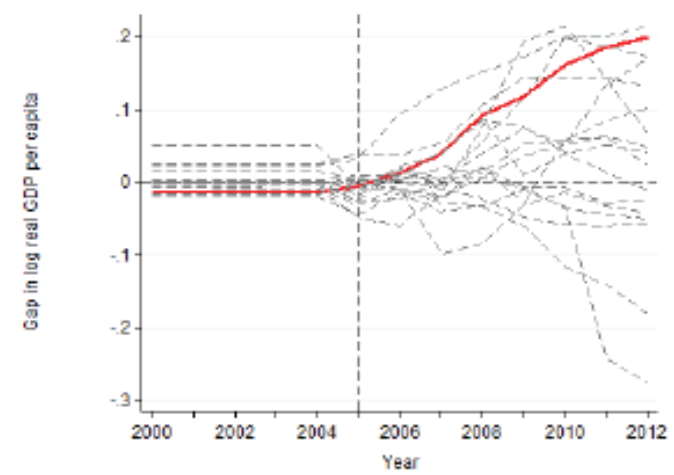

(d) Unemployment, Peru

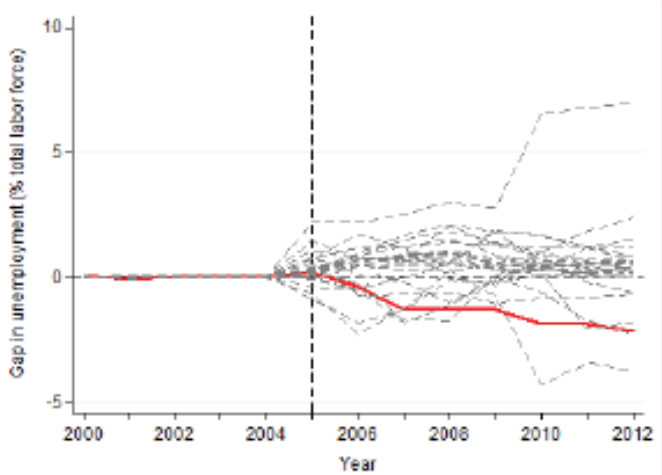

(f) Gini index, Peru

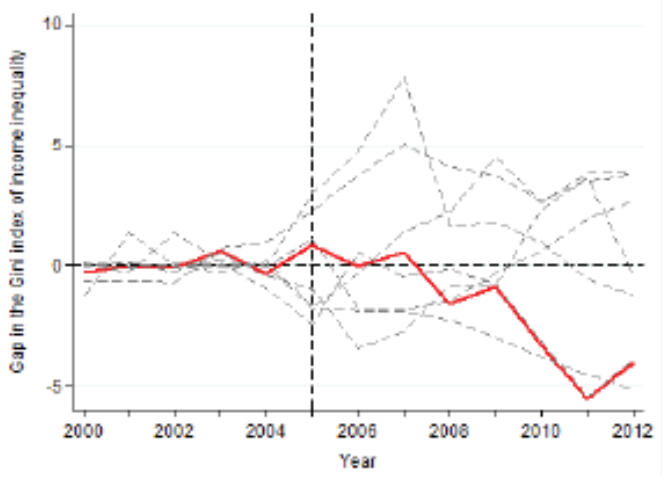

Notes: Authors' calculations based on WDI, FERDI, ICRG and Laeven and Valencia (2008, 2012). The solid red line represents outcome difference between the treated country and its synthetic control. The dotted grey lines depict the outcome difference between each of the treated country's potential controls and their respective synthetic control in placebo experiments. Only gaps such that the ratio of the placebo RMSPE to the "true" RMSPE is lower than the sample median are represented. The "true" surge country is included in the donor pool when conducting falsification tests. In all cases, the donor pool from which the synthetic control is constructed is restricted to the sample of emerging market and developing economies as defined by the IMF World Economic Outlook Database (October 2015). It further excludes countries with missing data for the outcome variable over the 5 years preceding the surge date and 7 years following it. The description and source of variables are provided in Table A3. 


\begin{tabular}{|c|c|}
\hline \multicolumn{2}{|l|}{ Panel A: Brazil } \\
\hline Real GDP per capita (log) & $\begin{array}{l}\text { Venezuela (43.3\%); Mexico (30.9\%); Jamaica (20.9\%); Malaysia (4.9\%). Other potential controls: Burkina Faso; Botswana; } \\
\text { Dominican Republic; Gabon; the Gambia; Guinea-Bissau; Guyana; Honduras; Indonesia; Sri Lanka; Mali; Mongolia; } \\
\text { Namibia; Niger; Nigeria; Philippines; Senegal; Sierra Leone; El Salvador; South Africa; Zimbabwe. }\end{array}$ \\
\hline Unemployment & $\begin{array}{l}\text { Indonesia (48.6\%); Fiji (36.2\%); South Africa (12.2\%); Moldova (3\%). Other potential controls: Armenia; Burundi; Benin; } \\
\text { Burkina Faso; Belize; Barbados; Brunei; Bhutan; Botswana; Central African Republic; Dominican Republic; Gabon; The } \\
\text { Gambia; Guinea-Bissau; Guyana; Honduras; Iraq; Jamaica; Kazakhstan; Cambodia; Sri Lanka; Lesotho; Mexico; Mali; } \\
\text { Mongolia; Mauritius; Malaysia; Niger; Nigeria; Nepal; Philippines; Sudan; Senegal; Sierra Leone; El Salvador; Swaziland; } \\
\text { Chad; Venezuela; Zimbabwe. }\end{array}$ \\
\hline Gini index & $\begin{array}{l}\text { Botswana (47.3\%); South Africa (31.6\%); El Salvador (10.8\%); Nigeria (10.3\%). Other potential controls: Burkina Faso; } \\
\text { Central African Republic; Dominican Republic; Guinea-Bissau; Honduras; Indonesia; Kazakhstan; Cambodia; Sri Lanka; } \\
\text { Lesotho; Moldova; Mexico; Mali; Mongolia; Malaysia; Niger; Nigeria; Nepal; Philippines; Senegal; Swaziland. }\end{array}$ \\
\hline \multicolumn{2}{|r|}{ e } \\
\hline Real GDP per capita (log) & 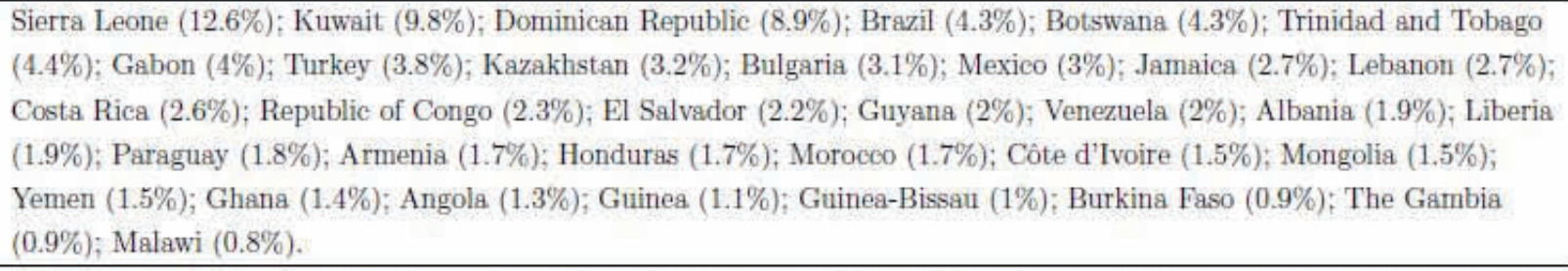 \\
\hline Unemployment & $\begin{array}{l}\text { Burkina Faso (58.3\%); Fiji (19.8\%); Kazakhstan (9.1\%); Yemen (6.8\%); Venezuela (6\%). Other potential controls: Angola; } \\
\text { Albania; Armenia; Benin; Bulgaria; The Bahamas; Brazil; Barabados; Botswana; Central African Republic; Côte d'Ivoire; } \\
\text { Republic of Congo; Costa Rica; Dominican Republic; Gabon; Ghana; The Gambia; Guinea-Bissau; Equatorial Guinea; } \\
\text { Guyana; Honduras; Iraq; Jamaica; Cambodia; Kuwait; Lesotho; Morocco; Mexico; Macedonia; Mongolia; Mauritania; } \\
\text { Malawi; Nepal; Paraguay; Qatar; Saudi Arabia; Sudan; Solomon Islands; Sierra Leone; El Salvador; Swaziland; Trinidad } \\
\text { and Tobago; Turkey. }\end{array}$ \\
\hline Gini index & $\begin{array}{l}\text { Paraguay }(41.5 \%) \text {; Honduras }(26.6 \%) \text {; Costa Rica }(23.7 \%) \text {; Albania }(8.2 \%) \text {. Other potential controls: Armenia; Bulgaria; } \\
\text { Brazil; Dominican Republic; Guinea; Kazakhstan; Cambodia; Mexico; Mongolia; El Salvador; Turkey. }\end{array}$ \\
\hline \multicolumn{2}{|c|}{$\begin{array}{l}\text { Notes: Authors' calculations based on WDI, FERDI, ICRG and Laeven and Valencia (2008, 2012). The donor pool from which the synthetic control is construeted is restrieted } \\
\text { to the sample of emerging market and developing economies as defined by the IMF World Economic Outlook Database (October 2015). It excludes countries with missing data } \\
\text { for the outcome variable over the } 5 \text { years preceding the surge date and } 7 \text { years following it. Control countrics selected by the algorithm to build the synthetie control are given } \\
\text { with individual weights in parentheses. Other potential controls are untreated eountries that were not selected. The description and source of variables are provided in Table } \\
\text { A3. }\end{array}$} \\
\hline
\end{tabular}


Table 7. Means of Covariates and Outcomes

\begin{tabular}{|c|c|c|c|c|}
\hline & \multicolumn{2}{|c|}{ Brazil } & \multicolumn{2}{|c|}{ Peru } \\
\hline & Treated & Synthetic & Treated & Synthetic \\
\hline \multicolumn{5}{|l|}{ Real GDP per capita (log) } \\
\hline Investment (\% GDP) & 18.69 & 22.77 & 17.18 & 18.76 \\
\hline Population growth & 0.02 & 0.02 & 0.01 & 0.02 \\
\hline Human assets index & 85.33 & 81.74 & 81.50 & 70.36 \\
\hline Government quality & 2.68 & 2.82 & 2.58 & 2.39 \\
\hline Crisis & 1.00 & 0.62 & 0.00 & 0.07 \\
\hline Pre-treatment real GDP per capita (log) & 9.06 & 9.06 & 8.15 & 8.16 \\
\hline Real GDP per capita (log) at $T_{0}+1$ & 9.08 & 9.06 & 8.32 & 8.30 \\
\hline Real GDP per capita $(\log )$ at $T_{0}+3$ & 9.09 & 8.98 & 8.46 & 8.37 \\
\hline Real GDP per capita $(\log )$ at $T_{0}+5$ & 9.15 & 9.10 & 8.53 & 8.37 \\
\hline Real GDP per capita $(\log )$ at $T_{0}+7$ & 9.23 & 9.19 & 8,62 & 8.42 \\
\hline RMSPE & \multicolumn{2}{|c|}{0.01} & \multicolumn{2}{|c|}{0.00} \\
\hline \multicolumn{5}{|l|}{ Unemployment (\% total labor force) } \\
\hline Population growth & 0.02 & 0.01 & 0.01 & 0.02 \\
\hline Human assets index & 85.33 & 79.55 & 81.50 & 46.34 \\
\hline Real GDP per capita (log) & 9.06 & 7.94 & 8.15 & 7.00 \\
\hline Inflation & 19.35 & 12.71 & 2.37 & 4.21 \\
\hline Urban population (\% total) & 79.05 & 43.70 & 73.85 & 33.07 \\
\hline Pre-treatment unemployment & 7.80 & 7.81 & 5.70 & 5.70 \\
\hline Unemployment at $T_{0}+1$ & 9.30 & 9.46 & 4.60 & 4.96 \\
\hline Unemployment at $T_{0}+3$ & 9.70 & 11.24 & 4.50 & 5.74 \\
\hline Unemployment at $T_{0}+5$ & 9.30 & 10.23 & 4.00 & 5.86 \\
\hline Unemployment at $T_{0}+7$ & 8.10 & 10.41 & 3.60 & 5.78 \\
\hline RMSPE & \multicolumn{2}{|c|}{0.13} & \multicolumn{2}{|c|}{0.06} \\
\hline \multicolumn{5}{|l|}{ Gini index of income inequality } \\
\hline Population growth & 0.02 & 0.02 & 0.01 & 0.02 \\
\hline Human assets index & 85.33 & 67.57 & 81.50 & 82.36 \\
\hline Real GDP per capita (log) & 9.06 & 8.33 & 8.15 & 7.97 \\
\hline Government spending (\% GDP) & 19.56 & 21.53 & 11.48 & 12.07 \\
\hline Gender gap in labor force participation & 64.45 & 75.08 & 73.02 & 55.70 \\
\hline Pre-treatment Gini index & 59.57 & 59.56 & 52.31 & 52.32 \\
\hline Gini index at $T_{0}+1$ & 59.33 & 59.03 & 51.67 & 51.71 \\
\hline Gini index at $T_{0}+3$ & 58.01 & 59.30 & 48.55 & 50.11 \\
\hline Gini index at $T_{0}+5$ & 56.64 & 59.25 & 46.21 & 49.53 \\
\hline Gini index at $T_{0}+7$ & 55.23 & 58.58 & 45.11 & 49.15 \\
\hline RMSPE & \multicolumn{2}{|c|}{0.05} & \multicolumn{2}{|c|}{0.33} \\
\hline \multicolumn{5}{|c|}{$\begin{array}{l}\text { Notes: Authors' calculations based on WDI, FERDI, ICRG and Laeven and Valencia (2008, 2012). The } \\
\text { donor pool from which the synthetic control is constructed is restricted to the sample of emerging market } \\
\text { and developing economies as defined by the IMF World Economic Outlook Database (October 2015). } \\
\text { It excludes countries with missing data for the outcome variable over the } 5 \text { years precoding the surge } \\
\text { date and } 7 \text { years following it. The pre-treatment outcome is the value of the outcome averaged over the } \\
\text { pre-treatement period to provide a simple reference, but the slgorithm minimizes the distance between } \\
\text { each yearly value of the outcome for the treated country and its synthetic counterpart. RMSPE: root } \\
\text { mean squared prediction error. The description and source of variables are provided in Table A3. }\end{array}$} \\
\hline
\end{tabular}


57. Summing up, export accelerations can have favorable impacts on the level and distribution of income. The case studies of Brazil and Peru demonstrate higher GDP per capita in the years following an export acceleration, although the results are not conclusive for Brazil. The results also highlight that surges in goods and services exports improve income distribution and labor market conditions in the countries where they occur through lower unemployment rate and reduced income inequality.

\section{Conclusion}

58. Using a large panel of 187 countries, this paper explores the correlates of export accelerations in goods and services for Latin America and the world. We find that lower macroeconomic uncertainty, improved quality of institutions, a depreciated exchange rate, agricultural reforms and participation in GVCs show up as strong predictors of the timing of export accelerations, irrespective of the sample and type of export considered. The paper also provides evidence of heterogeneous responses:

- Diversification matters for export transitions; but, while the positive effect materializes through the intensive margin of trade for the world, diversification at the extensive margin seems to be key to achieving high and sustained export growth in LAC. Results also suggest spillover effects from goods to services as product diversification positively correlates with surges in services exports.

- Lowering average applied tariff rates raises the likelihood of experiencing export accelerations through reduced tariffs on manufactures. In LAC, high tariffs on primary products undermine the occurrence of services export accelerations, possibly reflecting complementarities between services exports and goods imports in the region.

- Participation in GVCs is critical to triggering accelerations, and results are driven by growth in the foreign value-added content of exports. In contrast, LAC countries seem to strongly benefit from both downstream and upstream involvement in multi-stage trade process.

- Surges in services exports are more sensitive to investment growth and to the extent of liberalization of the electricity and telecommunication markets.

- In the case of LAC, services export accelerations are also preceded by growth in FDI inflows and domestic financial liberalization through banking sector reforms. In contrast, goods export surges respond to capital account openness.

59. The findings indicate that adopting policies geared towards promoting export growth is all the more important for LAC given the broadly large estimates we find for the region relative to the world sample. The illustrative case studies of Brazil and Peru further highlight the importance of implementing policies to bolster export growth by showing that post-acceleration years are characterized by higher GDP per capita and lower unemployment and income inequality. 


\section{REFERENCES}

Abadie, A., A. Diamond, and J. Hainmueller, 2010, "Synthetic Control Methods for Comparative Case Studies: Estimating the Effect of California's Tobacco Control Program," Journal of the American Statistical Association, 105(490), 493-505.

Abadie, A., A. Diamond, and J. Hainmueller, 2011, "Synth: An R Package for Synthetic Control Methods in Comparative Case Studies," Journal of Statistical Software, 42(13), 117.

Abadie, A., A. Diamond, and J. Hainmueller, 2015, "Comparative Politics and the Synthetic Control Method," American Journal of Political Science, 59(2), 495-510.

Abadie, A., and J. Gardeazabal, 2003, "The Economic Costs of Confict: A Case Study of the Basque Country," The American Economic Review, 93(1), 113-132.

Adhikari, B., R. A. Duval, B. Hu, and P. Loungani, 2016, "Can Reform Waves Turn the Tide? Some Case Studies Using the Synthetic Control Method," WP/16/171, International Monetary Fund.

Amin, M., and A. Mattoo, 2006, "Do Institutions Matter More for Services?," World Bank Policy Research Working Paper Series, 4032.

Amiti, M., and D. E. Weinstein, 2011, "Exports and Financial Shocks," Quarterly Journal of Economics, 126(4), 1841-1877.

Arnold, J. M., B. Javorcik, M. Lipscomb, and A. Mattoo, 2016, "Services Reform and Manufacturing Performance: Evidence from India,” Economic Journal, 126(590), 1-39.

Arnold, J. M., B. S. Javorcik, and A. Mattoo, 2011, "Does Services Liberalization Benefit Manufacturing Firms? Evidence from the Czech Republic," Journal of International Economics, 85(1), 136-146.

Arnold, J. M., A. Mattoo, and G. Narciso, 2008, "Services Inputs and Firm Productivity in Sub-Saharan Africa: Evidence from Firm-Level Data," Journal of African Economies, 17(4), 578-599.

Baier, S. L., and J. H. Bergstrand, 2007, "Do Free Trade Agreements Actually Increase Members' International Trade?," Journal of international Economics, 71(1), 72-95.

Barro, R. J., 1991, "Economic Growth in a Cross Section of Countries," Quarterly Journal of Economics, 106, 407-443.

Barro, R. J., 2000, "Inequality and Growth in a Panel of Countries," Journal of Economic Growth, 5(1), 5-32.

Bekaert, G., and C. R. Harvey, 2000, "Foreign Speculators and Emerging Equity Markets," The Journal of Finance, 55(2), 565-613.

Bekaert, G., C. R. Harvey, and C. Lundblad, 2005, "Does Financial Liberalization Spur Growth?," Journal of Financial Economics, 77(1), 3-55.

Ben-David, D., and D. H. Papell, 1998, "Slowdowns and Meltdowns: Postwar Growth Evidence from 74 Countries," Review of Economics and Statistics, 80(4), 561-571. 
Berg, A., J. D. Ostry, and J. Zettelmeyer, 2012, "What Makes Growth Sustained?," Journal of Development Economics, 98(2), 149-166.

Bergstrand, J. H., 1985, "The Gravity Equation in International Trade: Some Microeconomic Foundations and Empirical Evidence," Review of Economics and Statistics, pp. 474-481.

Bernard, A. B., J. B. Jensen, and R. Z. Lawrence, 1995, "Exporters, Jobs, and Wages in US Manufacturing: 1976-1987," Brookings Papers on Economic Activity. Microeconomics, 1995, 67-119.

Billmeier, A., and T. Nannicini, 2013, "Assessing Economic Liberalization Episodes: A Synthetic Control Approach," Review of Economics and Statistics, 95(3), 983-1001.

Cadot, O., C. Carrère, and V. Strauss-Kahn, 2011, "Export Diversification: What's Behind The Hump?," Review of Economics and Statistics, 93(2), 590-605.

Cadot, O., C. Carrère, and V. Strauss-Kahn, 2014, Trade and Employment: From Myths to Facts, Chapter 7, Trade Diversification: Drivers and Impacts, pp. 253-307. ILO.

Cadot, O., J. de Melo, P. Plane, L. Wagner, and M. T. Woldemichael, 2015, "Industrialization and Structural Change, Can Sub-Saharan Africa Develop without Factories?," Working Paper Ferdi, P143.

Cadot, O., A.-C. Disdier, M. Jaud, and A. Suwa-Eisenmann, 2015, "Big Hits in Export: Growing by Leaps and Bounds," Mimeo, Lausanne.

Carrère, C., and J. de Melo, 2012, "Fiscal Spending and Economic Growth: Some Stylized Facts," World Development, 40(9), 1750-1761.

Carrère, C., J. de Melo, and J. S. Wilson, 2009, “The Distance Effect and The Regionalization of The Trade of Low-Income Countries," CEPR Discussion Paper No. DP7458.

Cerra, V., and S. C. Saxena, 2008, "Growth Dynamics: The Myth of Economic Recovery," American Economic Review, 98(1), 439-457.

Chor, D., and K. Manova, 2012, "Off the Cliff and Back? Credit Conditions and International Trade During the Global Financial Crisis," Journal of International Economics,87(1), 117 133.

Dabla-Norris, E., K. Kochhar, N. Suphaphiphat, F. Ricka, and E. Tsounta, 2015, "Causes and Consequences of Income Inequality: A Global Perspective," IMF Staff Discussion Note, $\mathrm{SDN} / 15 / 43$.

Dell'Ariccia, G., D. Igan, L. Laeven, and H. Tong, 2012, "Policies for Macrofinancial Stability: How to Deal with Credit Booms," IMF Staff Discussion Note, SDN/12/06.

Dreher, A., 2006, "Does Globalization Affect Growth? Evidence from a New Index of Globalization," Applied Economics, 38(10), 1091-1110.

Dreher, A., N. Gaston, and P. Martens, 2008, Measuring Globalization: Gauging Its Consequences. Springer Science \& Business Media.

Ebeke, C., 2014, “Private Saving Accelerations," WP/14/223, International Monetary Fund.

Eichengreen, B., and P. Gupta, 2013, "The Real Exchange Rate and Export Growth: Are Services Different?," World Bank Policy Research Working Paper Series, 6629. 
Feenstra, R. C., R. Inklaar, and M. P. Timmer, 2015, "The Next Generation of the Penn World Table," American Economic Review, 105(10), 3150-3182.

Fernandes, A. M., C. Freund, and M. D. Pierola, 2016, "Exporter Behavior, Country Size and Stage of Development: Evidence from the Exporter Dynamics Database," Journal of Development Economics, 119, 121-137.

Fernandes, A. M., and C. Paunov, 2012, "Foreign Direct Investment in Services and Manufacturing Productivity: Evidence for Chile," Journal of Development Economics, 97(2), 305-321.

Frankel, J., E. Stein, and S.-J. Wei, 1995, "Trading Blocs and the Americas: The Natural, the Unnatural, and the Super-natural," Journal of Development Economics, 47(1), 61-95.

Frankel, J. A., and D. Romer, 1999, "Does Trade Cause Growth?," American Economic Review, pp. 379-399.

Frankel, J. A., E. Stein, and S.-J. Wei, 1997, Regional Trading Blocs in the World Economic System. Peterson Institute.

Freund, C., and M. D. Pierola, 2012, “Export Surges,” Journal of Development Economics, 97, 387-395.

Freund, C., and D. Weinhold, 2002, "The Internet and International Trade in Services," American Economic Review, 92(2), 236-240.

Fugazza, M., 2004, "Export Performance and Its Determinants: Supply and Demand Constraints," Policy Issues on International Trade and Commodities Study Series, (26).

Gonzales, C., S. Jain-Chandra, K. Kochhar, M. Newiak, and T. Zeinullayev, 2015, "Catalyst for Change: Empowering Women and Tackling Income Inequality," IMF Staff Discussion Note, SDN/15/20.

Guillaumont, P., and L.Wagner, 2012, "Aid and Growth Accelerations: Vulnerability Matters," Working paper no. 2012/31, United Nations University - World Institute for Development Economics Research.

Hannan, S. A., 2016, "The Impact of Trade Agreements: New Approach, New Insights," WP/16/117, International Monetary Fund.

Hausmann, R., L. Pritchett, and D. Rodrik, 2005, "Growth Accelerations," Journal of Economic Growth, 10(4), 303-329.

Henry, P. B., 2000, "Stock Market Liberalization, Economic Reform, and Emerging Market Equity Prices," The Journal of Finance, 55(2), 529-564.

Iacovone, L., and V. Zavacka, 2009, "Banking Crises and Exports: Lessons from the Past," World Bank Policy Research Working Paper Series, 5016.

International Monetary Fund, 2012, "The Liberalization and Management of Capital Flows: An Institutional View," November 2012.

International Monetary Fund, 2015, “Adjusting Under Pressure,” Regional Economic Outlook, Western Hemisphere, Chapter 4. Trade Integration in Latin America and the Caribbean: Hype, Hope and Reality, October 2015. 
International Monetary Fund, 2016, "Subdued Demand: Symptoms and Remedies," World Economic Outlook, Chapter 2. Global Trade: What's behind the Slowdown?, October 2016.

Jensen, J. B., 2008, "Trade in High-Tech Services," Journal of Industry, Competition and Trade, 8(3-4), 181-197.

Jerzmanowski, M., 2006, "Empirics of Hills, Plateaus, Mountains and Plains: A MarkovSwitching Approach to Growth," Journal of Development Economics, 81(2), 357-385.

Jones, B. F., and B. A. Olken, 2005, "Do Leaders Matter? National Leadership and Growth Since World War II," Quarterly Journal of Economics, 120(3).

Jones, B. F., and B. A. Olken, 2008, "The Anatomy of Start-Stop Growth," Review of Economics and Statistics, 90(3), $582\{587$.

Kaminsky, G. L., and C. M. Reinhart, 1999, "The Twin Crises: The Causes of Banking and Balance-of-Payments Problems," American Economic Review, 89(3), 473-500.

Kiendrebeogo, Y., 2013, “How Do Banking Crises Affect Bilateral Exports?,” WP/13/150, International Monetary Fund.

Kiendrebeogo, Y., and A. Minea, 2016, "Financial Factors and Manufacturing Exports: FirmLevel Evidence from Egypt," Journal of Development Studies, pp. 1-17.

King, G., and L. Zeng, 2001, "Logistic Regression in Rare Events Data,” Political Analysis, $9(2), 137-163$.

Koopman, R., W. Powers, Z. Wang, and S.-J. Wei, 2010, "Give Credit Where Credit Is Due: Tracing Value Added in Global Production Chains," Working Paper 16426, National Bureau of Economic Research.

Koopman, R., Z. Wang, and S.-J. Wei, 2014, "Tracing Value-Added and Double Counting in Gross Exports," American Economic Review, 104(2), 459-494.

Kuznets, S., 1955, "Economic Growth and Income Inequality," American Economic Review, $45(1), 1-28$.

Laeven, L., 2003, “Does Financial Liberalization Reduce Financing Constraints?," Financial Management, 21(1), 5-34.

Laeven, L., and F. Valencia, 2008, “Systemic Banking Crises: A New Database,” WP/08/224, International Monetary Fund.

Laeven, L., and F. Valencia, 2012, "Systemic Banking Crises Database: An Update," WP/12/163, International Monetary Fund.

Lanau, S., and P. Topalova, 2016, "The Impact of Product Market Reforms on Firm Productivity in Italy," WP/16/119, International Monetary Fund.

Lennon, C., 2009, "Trade in Services and Trade in Goods: Differences and Complementarities," WIIW Working paper 53, Vienna Institute of International Economics Studies, Vienna.

Lennon, C., D. Mirza, and G. Nicoletti, 2009, "Complementarity of Inputs across Countries in Services Trade," Annals of Economics and Statistics, (93/94), 183-205. 
Lenzen, M., K. Kanemoto, D. Moran, and A. Geschke, 2012, "Mapping the Structure of the World Economy," Environmental Science and Technology, 46(15), 8374-8381.

Lenzen, M., D. Moran, K. Kanemoto, and A. Geschke, 2013, "Building Eora: A Global MultiRegion Input-Output Database at High Country and Sector Resolution," Economic Systems Research, 25(1), 20-49.

Manova, K., 2008, "Credit Constraints, Equity Market Liberalizations and International Trade," Journal of International Economics, 76(1), 33-47.

Manova, K., S.-J. Wei, and Z. Zhang, 2015, "Firm Exports and Multinational Activity under Credit Constraints," Review of Economics and Statistics, 97(3), 574-588.

Marshall, M. G., and K. Jaggers, 2002, "Polity IV Dataset," College Park, MD: Center for International Development and Conflict Management, University of Maryland.

Mendoza, E. G., and M. E. Terrones, 2012, "An Anatomy of Credit Booms and Their Demise," Journal Economía Chilena, 15(2), 04-32, Central Bank of Chile.

Mincer, J., 1958, "Investment in Human Capital and Personal Income Distribution," Journal of Political Economy, 66(4), 281-302.

Minetti, R., and S. C. Zhu, 2011, "Credit Constraints and Firm Export: Microeconomic Evidence from Italy," Journal of International Economics, 83(2), 109-125.

Muûls, M., 2015, "Exporters, Importers and Credit Constraints," Journal of International Economics, 95(2), 333-343.

Novta, N., and F. Rodrigues Bastos, 2016, "Eora Guide for IMF Desk Work," Forthcoming, International Monetary Fund.

Nunn, N., 2007, "Relationship-Specificity, Incomplete Contracts, and the Pattern of Trade," Quarterly Journal of Economics, pp. 569-600.

Ostry, J. D., A. R. Ghosh, K. Habermeier, M. Chamon, M. S. Qureshi, and D. B. S. Reinhardt, 2010, "Capital Inflows: The Role of Controls," IMF Staff Position Note, SPN/10/04.

Perotti, R., 1992, "Income Distribution, Politics and Growth," American Economic Review, 82(2), 311-316.

Prati, A., M. G. Onorato, and C. Papageorgiou, 2013, "Which Reforms Work and Under What Institutional Environment? Evidence from A New Dataset on Structural Reforms," Review of Economics and Statistics, 95(3), 946-968.

Pritchett, L., 2000, "Understanding Patterns of Economic Growth: Searching for Hills among Plateaus, Mountains, and Plains," World Bank Economic Review, 14(2), 221-250.

Rancière, R., and A. Tornell, 2015, "Financial Liberalization, Debt Mismatch, Allocative Efficiency, and Growth," American Economic Journal: Macroeconomics, Forthcoming.

Rancière, R., A. Tornell, and F. Westermann, 2008, "Financial Liberalization,” New Palgrave Dictionary of Economics.

Ranciere, R., A. Tornell, and F. Westermann, 2008, "Systemic Crises and Growth," Quarterly Journal of Economics, 123(1), 359-406.

Rodrik, D., 2000, "Saving Transitions," World Bank Economic Review, 14(3), 481-507. 
Rodrik, D., 2008, "The Real Exchange Rate and Economic Growth," Brookings Papers on Economic Activity, 2008(2), 365-412.

Sachs, J. D., and A. Warner, 1995, "Economic Reform and the Process of Global Integration," Brookings Papers on Economic Activity, 1995(1), 1-118.

Sáez, S., D. Taglioni, E. Van der Marel, C. H. Hollweg, and V. Zavacka, 2015, Valuing Services in Trade: A Toolkit for Competitiveness Diagnostics. World Bank Publications.

Schularick, M., and A. M. Taylor, 2012, "Credit Booms Gone Bust: Monetary Policy, Leverage Cycles, and Financial Crises, 1870-2008," American Economic Review, 102(2), 1029-61.

UNCTAD, 2015, "Global Value Chains and Development: Investment and Value Added Trade in the Global Economy," Advanced Unedited Version.

Van der Marel, E., 2012, "Trade in Services and TFP: The Role of Regulation," The World Economy, 35(11), 1530-1558.

Wacziarg, R., and K. H. Welch, 2008, "Trade Liberalization and Growth: New Evidence," World Bank Economic Review, 22(2), 187-231.

Winters, L. A., 2004, "Trade Liberalization and Economic Performance: An Overview," The Economic Journal, 114(493), F4-F21. 


\section{APPENDIX I.}

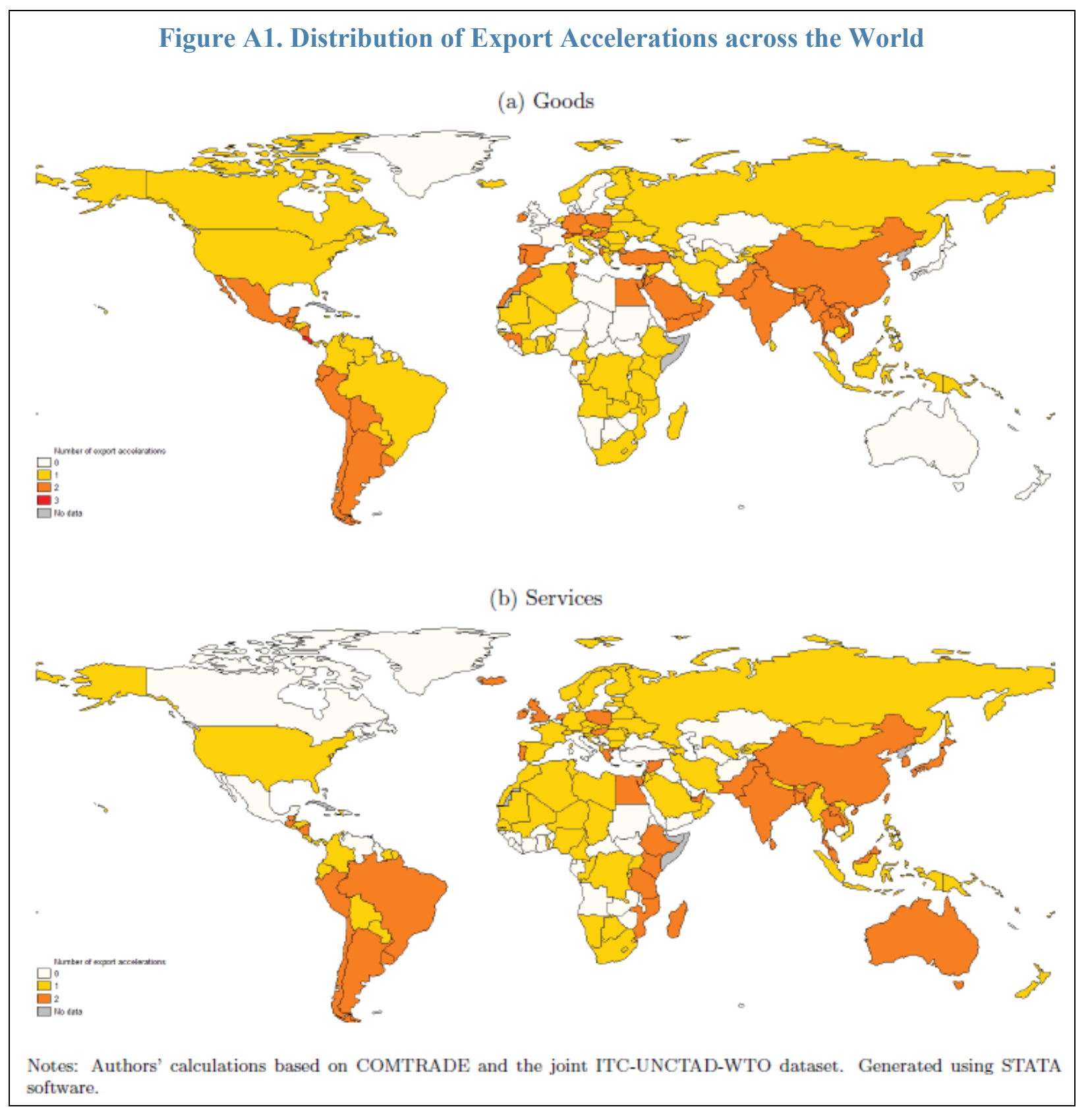


Table A1. Export Acceleration Dates in Advanced Economies

\begin{tabular}{|c|c|c|}
\hline \multirow{2}{*}{ Country } & \multicolumn{2}{|c|}{ Export Acceleration Dates } \\
\hline & Goods & Services \\
\hline Australia & $\ldots$ & $1988 ; 2003$ \\
\hline Austria & $1986 ; 2002$ & 2003 \\
\hline Belgium & $\ldots$ & $\ldots$ \\
\hline Canada & 1993 &..+ \\
\hline Cyprus & 2004 & 2002 \\
\hline Czech Republic & 2002 & 2004 \\
\hline Denmark & $\cdots$ & 1999 \\
\hline Estonia & $\ldots$ & 2003 \\
\hline Finland & 1994 & 2001 \\
\hline France & $\ldots$ & 2003 \\
\hline Germany & $1986 ; 1999$ & 2002 \\
\hline Greece & 2003 & $1990 ; 1999$ \\
\hline Hong Kong, China & 1986 & $1988 ; 2002$ \\
\hline Iceland & 1986 & $1995 ; 2003$ \\
\hline Ireland & $1986 ; 1994$ & $1988 ; 1996$ \\
\hline Israel & $1986 ; 1994$ & 1992 \\
\hline Italy & 1986 & ... \\
\hline Japan & $\ldots$ & $1988 ; 2004$ \\
\hline Korea, Rep. & $1986 ; 1999$ & $1988 ; 2003$ \\
\hline Latvia & 2003 & 2003 \\
\hline Lithuania & $\ldots$ & $\ldots$ \\
\hline Luxembourg & $\ldots$ & $\ldots$ \\
\hline Malta & $1987 ; 1998$ & 2003 \\
\hline Netherlands & 1998 & $1988 ; 2003$ \\
\hline New Zealand & $\ldots$ & 2002 \\
\hline Norway & 2004 & 1999 \\
\hline Portugal & $1986 ; 2002$ & $1988 ; 2002$ \\
\hline Singapore & $1986 ; 2004$ & $1990 ; 2003$ \\
\hline Slovak Republic & 2001 & 2003 \\
\hline Slovenia & 2002 & 2003 \\
\hline Spain & $1986 ; 2002$ & 1998 \\
\hline Sweden & $\ldots$ & 1998 \\
\hline Switzerland & $1986 ; 2003$ & 2003 \\
\hline Taiwan & 1999 & $1988 ; 2006$ \\
\hline United Kingdom & $\ldots$ & $1990 ; 2002$ \\
\hline United States & 1987 & 2004 \\
\hline
\end{tabular}

Notes: "..." means no acceleration date was identified. Advanced economies are identified based on the IMF World Economic Outlook database (October 2015). All countries are classified as high-income economies in the World Bank's 2013 income classification as their GNI per capita exceeds $\$ 12,745$. 


\begin{tabular}{|c|c|c|c|c|}
\hline \multirow[t]{2}{*}{ Country } & \multicolumn{2}{|c|}{ Export Acceleration Dates } & \multirow{2}{*}{$\begin{array}{l}\text { Main Source of } \\
\text { Export Earnings }\end{array}$} & \multirow{2}{*}{$\begin{array}{l}\text { Income } \\
\text { Group }\end{array}$} \\
\hline & Goods & Services & & \\
\hline \multicolumn{5}{|c|}{ Latin America and the Caribbean } \\
\hline \multicolumn{5}{|l|}{ North America } \\
\hline Mexico & $1986 ; 1994$ & $\ldots$ & Manufacturing & Upper-middle \\
\hline \multicolumn{5}{|l|}{ South America } \\
\hline Argentina & $1988 ; 2004$ & $1989 ; 2005$ & Primary products & Upper-middle \\
\hline Bolivia & $1989 ; 2003$ & 2003 & Fuel & Lower-middle \\
\hline Brazil & 2000 & $1989 ; 1998$ & Diversified & Upper-middle \\
\hline Chile & $1986 ; 2000$ & $1989 ; 2002$ & Primary products & High \\
\hline Colombia & 2004 & 2005 & Fuel & Upper-middle \\
\hline Ecuador & $1990 ; 2005$ & 2007 & Fuel & Upper-middle \\
\hline Guyana & $\ldots$ & $\ldots$ & Primary products & Lower-middle \\
\hline Paraguay & 2004 & 1988 & Primary products & Lower-middle \\
\hline Peru & $1988 ; 2004$ & $1994 ; 2005$ & Diversified & Upper-middle \\
\hline Suriname & 2006 & 2004 & Primary products & Upper-middle \\
\hline Uruguay & $1986 ; 2005$ & $1991 ; 2007$ & Primary products & High \\
\hline Venezuela & 1988 & $\ldots$ & Fuel & Upper-middle \\
\hline \multicolumn{5}{|l|}{ Central America } \\
\hline Belize & 1992 & 2001 & Diversified & Upper-middle \\
\hline Costa Rica & $1986 ; 1994 ; 2007$ & 1988 & Diversified & Upper-middle \\
\hline El Salvador & 1994 & 1997 & Diversified & Lower-middle \\
\hline Guatemala & $1991 ; 2005$ & $1989 ; 2001$ & Diversified & Lower-middle \\
\hline Honduras & 1992 & 1991 & Services & Lower-middle \\
\hline Nicaragua & $1994 ; 2004$ & $1991 ; 2003$ & Diversified & Lower-middle \\
\hline Panama & 2005 & 2002 & Services & Upper-middle \\
\hline \multicolumn{5}{|l|}{ The Caribbean } \\
\hline Antigua and Barbuda & 2000 & $\cdots$ & Services & High \\
\hline Bahamas, The & $1986 ; 2001$ & $\ldots$ & Services & High \\
\hline Barbados & $\ldots$ & $\ldots$ & Services & High \\
\hline Dominica & 1987 & $\ldots$ & Services & Upper-middle \\
\hline Dominican Republic & 1988 & $\ldots$ & Services & Upper-middle \\
\hline Grenada & $\cdots$ & $\ldots$ & Services & Upper-middle \\
\hline Haiti & $\ldots$ & 2007 & Services & Low \\
\hline Jamaica & 1986 & $\ldots$ & Services & Upper-middle \\
\hline St. Kitts and Nevis & $\ldots$ & $\ldots$ & Services & High \\
\hline St. Lucia & $\ldots$ & $\ldots$ & Services & Upper-middle \\
\hline St. Vincent \& the Grenadines & $\ldots$ & 1995 & Services & Upper-middle \\
\hline Trinidad and Tobago & $1988 ; 2000$ & $\ldots$ & Fuel & High \\
\hline
\end{tabular}


Table A2: Export Acceleration Dates in Emerging Market and Developing Countries (Continued)

\begin{tabular}{|c|c|c|c|c|}
\hline \multirow[t]{2}{*}{ Country } & \multicolumn{2}{|c|}{ Export Acceleration Dates } & \multirow{2}{*}{$\begin{array}{l}\text { Main Source of } \\
\text { Export Earnings }\end{array}$} & \multirow[t]{2}{*}{ Income Group } \\
\hline & Goods & Services & & \\
\hline \multicolumn{5}{|c|}{ Commonwealth of Independent States } \\
\hline Armenia & $\ldots$ & $\ldots$ & Services & Lower-middle \\
\hline Azerbaijan & 2005 & 2003 & Fuel & Upper-middle \\
\hline Belarus & 2003 & 2004 & Diversified & Upper-middle \\
\hline Georgia & 2004 & 2006 & Services & Lower-middle \\
\hline Kazakhstan & $\ldots$ & $\ldots$ & Fuel & Upper-middle \\
\hline Kyrgyz Republic & 2005 & 2003 & Services & Lower-middle \\
\hline Moldova & $\ldots$ & 2003 & Diversified & Lower-middle \\
\hline Russia & 2000 & 2004 & Fuel & High \\
\hline Tajikistan & 2004 & $\ldots$ & Services & Low \\
\hline Turkmenistan & 2004 & $\ldots$ & Fuel & Upper-middle \\
\hline Ukraine & 2004 & 2004 & Diversified & Lower-middle \\
\hline Uzbekistan & $\ldots$ & 2003 & Primary products & Lower-middle \\
\hline \multicolumn{5}{|c|}{ Emerging and Developing Asia } \\
\hline Bangladesh & $1987 ; 2003$ & $1988 ; 2003$ & Manufacturing & Low \\
\hline Bhutan & 1992 & 2004 & Diversified & Lower-middle \\
\hline Brunei & $\ldots$ & 2005 & Fuel & High \\
\hline Cambodia & 1987 & $\ldots$ & Manufacturing & Low \\
\hline China & $1986 ; 2000$ & $1990 ; 2001$ & Manufacturing & Upper-middle \\
\hline East Timor & $\ldots$ & $\ldots$ & Fuel & Lower-middle \\
\hline Fiji & 1989 & 1989 & Services & Upper-middle \\
\hline India & $1987 ; 2002$ & $1994 ; 2002$ & Diversified & Lower-middle \\
\hline Indonesia & 1987 & 2004 & Diversified & Lower-middle \\
\hline Kiribati & $\ldots$ & $\ldots$ & Services & Lower-middle \\
\hline Lao PDR & $1988 ; 2007$ & $1992 ; 2005$ & Diversified & Lower-middle \\
\hline Malaysia & 1987 & $1988 ; 2005$ & Manufacturing & Upper-middle \\
\hline Maldives & $\ldots$ & $1989 ; 2006$ & Services & Upper-middle \\
\hline Marshall Islands & $\ldots$ & $\ldots$ & Diversified & Upper-middle \\
\hline Micronesia, Fed. Sts. & $\ldots$ & $\ldots$ & Diversified & Lower-middle \\
\hline Mongolia & 1993 & 1998 & Primary products & Lower-middle \\
\hline Myanmar & $1990 ; 2007$ & 1992 & Diversified & Low \\
\hline Nepal & $\ldots$ & 1992 & Services & Low \\
\hline Palau & $\ldots$ & $\ldots$ & Services & Upper-middle \\
\hline Papua New Guinea & 2005 & 1989 & Primary products & Lower-middle \\
\hline Philippines & 1988 & 2007 & Services & Lower-middle \\
\hline Samoa & 1992 & 2003 & Services & Lower-middle \\
\hline Solomon Islands & 2006 & 1988 & Primary products & Lower-middle \\
\hline Sri Lanka & 1986 & $1990 ; 2007$ & Diversified & Lower-middle \\
\hline
\end{tabular}




\begin{tabular}{|c|c|c|c|c|}
\hline \multirow[t]{2}{*}{ Country } & \multicolumn{2}{|c|}{ Export Acceleration Dates } & \multirow{2}{*}{$\begin{array}{l}\text { Main Source of } \\
\text { Export Earnings }\end{array}$} & \multirow[t]{2}{*}{ Income Group } \\
\hline & Goods & Services & & \\
\hline Thailand & $1986 ; 2003$ & $1988 ; 2005$ & Manufacturing & Upper-middle \\
\hline Tonga & $\ldots$ & $\ldots$ & Services & Upper-middle \\
\hline Tuvalu & $\ldots$ & $\ldots$ & Primary products & Upper-middle \\
\hline Vanuatu & 2004 & $1990 ; 2007$ & Services & Lower-middle \\
\hline Vietnam & $1987 ; 2002$ & 2003 & Manufacturing & Lower-middle \\
\hline \multicolumn{5}{|c|}{ Emerging and Developing Europe } \\
\hline Albania & $1995 ; 2003$ & 1993 & Services & Upper-middle \\
\hline Bosnia and Herzegovina & $\ldots$ & $\ldots$ & Services & Upper-middle \\
\hline Bulgaria & 2001 & 1998 & Diversified & Upper-middle \\
\hline Croatia & 2003 & 2001 & Services & High \\
\hline Hungary & $1988 ; 1996$ & $1990 ; 2002$ & Manufacturing & Upper-middle \\
\hline Macedonia, FYR & 2004 & $\ldots$ & Services & Upper-middle \\
\hline Montenegro & $\ldots$ & $\ldots$ & Services & Upper-middle \\
\hline Poland & $1988 ; 1998$ & $1989 ; 2004$ & Manufacturing & High \\
\hline Romania & 1995 & 2000 & Manufacturing & Upper-middle \\
\hline Serbia & 2004 & $\ldots$ & Diversified & Upper-middle \\
\hline Turkey & $1986 ; 1998$ & $\ldots$ & Manufacturing & Upper-middle \\
\hline \multicolumn{5}{|c|}{ Middle East, North Africa, Afghanistan and Pakistan } \\
\hline Afghanistan & $\ldots$ & $\ldots$ & Primary products & Low \\
\hline Algeria & 2003 & 2002 & Fuel & Upper-middle \\
\hline Bahrain & 2000 & 2002 & Fuel & High \\
\hline Djibouti & $\ldots$ & $\ldots$ & Services & Lower-middle \\
\hline Egypt, Arab Rep. & $1989 ; 2000$ & $1988 ; 2003$ & Services & Lower-middle \\
\hline Sudan & $\ldots$ & $\ldots$ & Primary products & Lower-middle \\
\hline Iran, Islamic Rep. & 1999 & 2001 & Fuel & Upper-middle \\
\hline Iraq & $\ldots$ & $\ldots$ & Fuel & Upper-middle \\
\hline Jordan & $1984 ; 1998$ & 2005 & Services & Upper-middle \\
\hline Kuwait & 1998 & $\ldots$ & Fuel & High \\
\hline Lebanon & 1999 & $\ldots$ & Services & Upper-middle \\
\hline Libya & $\ldots$ & 2000 & Fuel & Upper-middle \\
\hline Mauritania & 2005 & 1999 & Primary products & Lower-middle \\
\hline Morocco & $1986 ; 2002$ & 1996 & Services & Lower-middle \\
\hline Oman & $1988 ; 2003$ & 2006 & Fuel & High \\
\hline Pakistan & $1986 ; 2002$ & $1989 ; 2002$ & Manufacturing & Lower-middle \\
\hline Qatar & 1997 & $\ldots$ & Fuel & High \\
\hline Saudi Arabia & $1986 ; 2000$ & 1997 & Fuel & High \\
\hline Syrian Arab Republic & 1990 & $1989 ; 2004$ & Diversified & Lower-middle \\
\hline Tunisia & $1987 ; 2002$ & 2004 & Manufacturing & Upper-middle \\
\hline
\end{tabular}




\begin{tabular}{|c|c|c|c|c|}
\hline \multirow[t]{2}{*}{ Country } & \multicolumn{2}{|c|}{ Export Acceleration Dates } & \multirow{2}{*}{$\begin{array}{l}\text { Main Source of } \\
\text { Export Earnings }\end{array}$} & \multirow[t]{2}{*}{ Income Group } \\
\hline & Goods & Services & & \\
\hline United Arab Emirates & 1989 & $1990 ; 2001$ & Fuel & High \\
\hline Yemen & $1987 ; 1998$ & $\ldots$ & Fuel & Lower-middle \\
\hline \multicolumn{5}{|c|}{ Sub-Saharan Africa } \\
\hline Angola & 2007 & $\ldots$ & Fuel & Upper-middle \\
\hline Benin & 1994 & $\ldots$ & Diversified & Low \\
\hline Botswana & $\ldots$ & 1999 & Manufacturing & Upper-middle \\
\hline Burkina Faso & $\ldots$ & $\ldots$ & Primary products & Low \\
\hline Burundi & $\ldots$ & 2004 & Primary products & Low \\
\hline Cabo Verde & 2006 & $1994 ; 2002$ & Services & Lower-middle \\
\hline Cameroon & 2005 & 2000 & Diversified & Lower-middle \\
\hline Central African Republic & $\ldots$ & $\ldots$ & Primary products & Low \\
\hline Chad & $\ldots$ & 2003 & Fuel & Low \\
\hline Comoros & 2001 & $\ldots$ & Services & Low \\
\hline Congo, Dem. Rep. & 1999 & 2003 & Primary products & Low \\
\hline Congo, Rep. & 2003 & 1999 & Fuel & Lower-middle \\
\hline Côte d'Ivoire & 2007 & $\ldots$ & Primary products & Lower-middle \\
\hline Equatorial Guinea & $1995 ; 2005$ & $\ldots$ & Fuel & High \\
\hline Eritrea & $\ldots$ & $\ldots$ & Primary products & Low \\
\hline Ethiopia & 2005 & $1996 ; 2004$ & Services & Low \\
\hline Gabon & $\ldots$ & $\ldots$ & Fuel & Upper-middle \\
\hline Gambia, The & 2008 & $\ldots$ & Services & Low \\
\hline Ghana & 2003 & $\ldots$ & Diversified & Lower-middle \\
\hline Guinea & $1987 ; 1995$ & $\ldots$ & Primary products & Low \\
\hline Guinea-Bissau & 1993 & $\ldots$ & Primary products & Low \\
\hline Kenya & 2004 & $1988 ; 2007$ & Diversified & Low \\
\hline Lesotho & $\ldots$ & $\ldots$ & Manufacturing & Lower-middle \\
\hline Liberia & $\ldots$ & $\ldots$ & Primary products & Low \\
\hline Madagascar & 1998 & $1988 ; 2004$ & Diversified & Low \\
\hline Malawi & 2007 & $\ldots$ & Primary products & Low \\
\hline Mali & 1989 & 2001 & Primary products & Low \\
\hline Mauritius & 1986 & 2003 & Services & Upper-middle \\
\hline Mozambique & 2004 & $1991 ; 2006$ & Diversified & Low \\
\hline Namibia & $\ldots$ & 2006 & Diversified & Upper-middle \\
\hline Niger & $\ldots$ & 2001 & Primary products & Low \\
\hline Nigeria & $\ldots$ & 2000 & Fuel & Lower-middle \\
\hline Rwanda & 2005 & 2003 & Services & Low \\
\hline São Tomé and Príncipe & 1997 & $\ldots$ & Services & Lower-middle \\
\hline Senegal & $\ldots$ & 2003 & Services & Lower-middle \\
\hline
\end{tabular}




\begin{tabular}{|c|c|c|c|c|}
\hline \multirow[t]{2}{*}{ Country } & \multicolumn{2}{|c|}{ Export Acceleration Dates } & \multirow{2}{*}{$\begin{array}{l}\text { Main Source of } \\
\text { Export Earnings }\end{array}$} & \multirow[t]{2}{*}{ Income Group } \\
\hline & Goods & Services & & \\
\hline Seychelles & 1999 & $\ldots$ & Diversified & Upper-middle \\
\hline Sierra Leone & $\ldots$ & $\ldots$ & Primary products & Low \\
\hline South Africa & 1992 & 2003 & Primary products & Upper-middle \\
\hline South Sudan & $\ldots$ & $\ldots$ & Fuel & Lower-middle \\
\hline Swaziland & $\ldots$ & 1988 & Manufacturing & Lower-middle \\
\hline Tanzania & 2003 & $1991 ; 2001$ & Diversified & Low \\
\hline Togo & 1997 & 2004 & Diversified & Low \\
\hline Uganda & 2005 & 2003 & Services & Low \\
\hline Zambia & 2004 & $\ldots$ & Primary products & Lower-middle \\
\hline Zimbabwe & $\ldots$ & $\ldots$ & Diversified & Low \\
\hline
\end{tabular}

Notes: "..." means no acceleration date was identified. The regional and analytical breakdowns of emerging market and developing countries are taken from the IMF World Economic Outlook database (October 2015). The analytical criterion Main Source of Export Earnings distinguishes between categories Fuel (SITC section 3) and Non-Fuel (SITC sections 0, 1, 2, 4 and division 68). Economies are categorized into one of these groups when their main source of export earnings exceeded $50 \%$ of total exports on average between 2010 and 2014. Services refer to Services, income, transfers. Income Group reflects the World Bank's 2013 income classification (FY 2015 ) based on per capita gross national income (GNI) with the following groups: (i) low-income: $\$ \$ 1,045$; (ii) lower-middle-income: $\$ 1,046$ to $\$ 4,125$; (iii) upper-middle-income: $\$ 4,126$ to $\$ 12,745$; (iv) high-income: $>\$ 12,745$. 


\begin{tabular}{|c|c|c|}
\hline \multicolumn{3}{|c|}{ Table A3: Description and Source of Variables } \\
\hline Variable & Description & Source \\
\hline Log goods exports & Log merchandise exports (constant $2010 \$$ ) & Calculated from COMTRADE (SITC rev.2) and WDI ${ }^{32}$ \\
\hline Goods export growth & Log difference of real merchandise exports & Calculated from COMTRADE (SITC rev.2) and WDI \\
\hline Log services exports & Log Services exports (constant $2010 \$$ ) & Calculated from WTO/UNCTAD/ITC and WDI ${ }^{93}$ \\
\hline Services export growth & Log difference of real services exports & Calculated from WTO/UNCTAD/ITC and WDI \\
\hline Log real GDP cap. & Log GDP per capita (constant 2010 US\$) & WDI \\
\hline Log population & Log total population & WDI \\
\hline Market access & $\begin{array}{l}\text { Sum of all the economic integration agreements (PTA, FTA, Customs Union, } \\
\text { Common Market, Economic Union) a country participates in, weighted by partners' } \\
\text { market size as measured by GDP. }\end{array}$ & Calculated from J. Bergstrand's database and WDI ${ }^{34}$ \\
\hline$\Delta$ Investment & Gross fixed capital formation ( $\%$ of GDP), 3-year change & WDI \\
\hline REER volatility & Standard deviation of the annual REER over the past 5 years & Calculated from IMF IFS \\
\hline$\Delta$ Infrastructure & $\begin{array}{l}\text { Quality of infrastructure index, } 3 \text {-year change. Simple mean of the number of main } \\
\text { telephone lines per } 1000 \text { workers, the length of the road network, the length of the } \\
\text { railway network (both in km per sq. km. of land area) and the share of paved roads } \\
\text { in total roads (quality in the service of transports) }\end{array}$ & Carrère, de Melo, and Wilson (2009) \\
\hline Quality of government & Mean value of Corruption, Law and Order and Bureaucracy Quality variables. & ICRG $^{36}$ \\
\hline Polity 2 & Degree of democracy $y^{37}$ & Polity IV database by Marshall and Jaggers (2002) \\
\hline Secondary education & Secondary school enrollment ( $\%$ gross) & WDI \\
\hline Log REER index & Real effective exchange rate index $(100=2010)^{38}$ & IMF IFS \\
\hline Log RER & Log RER at PPP 39 & Calculated based on Rodrik (2008) and \\
\hline RER misalignment & Difference between log RER at PPP and the Balassa-Samuelson adjusted RER. ${ }^{40}$ & from PWT8.0 (Feenstra, Inklaar, and Timmer, 2015) \\
\hline Theil index & Total theil index ${ }^{41}$ & IMF Diversification Toolkit \\
\hline Theil index, intensive margin & Theil, intensive margin & IMF Diversification Toolkit \\
\hline Theil index, extensive margin & Theil, extensive margin & IMF Diversification Toolkit \\
\hline$\Delta$ Trade openness & Trade in goods and services ( $\%$ of GDP), 3-year change & Calculated from WDI \\
\hline $\begin{array}{l}{ }^{32} \text { Exports exclude fuels (SII } \\
{ }^{33} \text { Deflated using US CPI inc } \\
{ }^{34} \text { The database records the } \\
\text { country pairing was assigned a } \\
{ }^{35} \text { Prior to computing the in } \\
{ }^{36} \text { Scales from } 0 \text { to } 1 \text {, higher } \\
{ }^{37}-10 \text { strongly autocratic; }+ \\
{ }^{38} \text { Higher values indicate an } \\
{ }^{39} \text { Higher values indicate an } \\
{ }^{40} \text { The Balassa-Samuelson ad } \\
{ }^{41} \text { Higher values indicate low }\end{array}$ & $\begin{array}{l}\text { ) and minerals (SITC divisions } 27,28 \text { and } 68 \text { ). Deflated using US CPI index ( } 100=2 \\
2000 \text { ). } \\
\text { ategration of bilateral country pairings for } 195 \text { countries annually from } 1950 \text { through } \\
\text { from } 0 \text { to } 6 \text {. We convert this code into a } 0 / 1 \text { dummy. } \\
\text { es are normalized so that they have the same mean of } 1 \text {. } \\
\text { cate better quality of government (lower political risk). } \\
\text { democratic } \\
\text { a. } \\
\text { is the log of the fitted (predicted) value from the regression of log RER at PPP on rea } \\
\text { ation. }\end{array}$ & Depending on the level of economic integration, a \\
\hline
\end{tabular}




\begin{tabular}{|c|c|c|}
\hline \multicolumn{3}{|c|}{ Table A3: Description and Source of Variables (Continued) } \\
\hline Variable & Description & Source \\
\hline Tariffs & Average applied tariff rates, all products (\%) & WDI \\
\hline Tariffs, manufactures & Average applied tariff rates, manufactured products (\%) & WDI \\
\hline Tariffs, primary products & Average applied tariff rates, primary products (\%) & WDI \\
\hline Agriculture index & $\begin{array}{l}\text { Degree of public intervention in the market of the main agricultural export } \\
\text { commodity, with } 4 \text { degrees: (i) maximum (public monopoly or monopsony in } \\
\text { production, transportation or marketing); (ii) high (administered prices); } \\
\text { (iii) moderate (public ownership in relevant producers, concession requirements; } \\
\text { (iv) no intervention }\end{array}$ & Prati, Onorato, and Papageorgiou (2013) ${ }^{42}$ \\
\hline Electricity \& Telecoms index & $\begin{array}{l}\text { Degree of competition and liberalization and quality of regulation in the } \\
\text { electricity and telecom markets. Elecricity: (i) degree of unbundling of generation, } \\
\text { transmission, and distribution; (ii) whether a regulator other than government } \\
\text { has been established; (iii) whether the wholesale market has been liberalized. } \\
\text { Telecom: (i) the degree of competition in local services; (ii) whether a regulator } \\
\text { other than government has been established; (iii) the degree of liberalization of } \\
\text { interconnection changes. }\end{array}$ & Prati, Onorato, and Papageorgiou (2013) \\
\hline Financial liberalization & Reforms in the banking sector and securities markets & Prati, Onorato, and Papageorgiou (2013) \\
\hline Banking sector reform & $\begin{array}{l}\text { (i) interest rate controls (floors or ceilings); (ii) credit controls (directed credit, } \\
\text { subsidized lending); (iii) competitition restrictions (limits on branches and entry } \\
\text { barriers in the banking sector (licensing requirements or limits on foreign banks); } \\
\text { (iv) degree of state ownership; (v) quality of banking supervision \& regulation } \\
\text { (power of independence of bank supervisors, adoption of a Basel I capital } \\
\text { adequacy ratio, framework for bank inspections }\end{array}$ & Prati, Onorato, and Papageorgiou (2013) \\
\hline Securities market reform & $\begin{array}{l}\text { Degree of liberalization of securities markets: policies to encourage the } \\
\text { development of bond and equity markets, to permit access of the domestic } \\
\text { stock market to foreigners. }\end{array}$ & Prati, Onorato, and Papageorgiou (2013) \\
\hline Capital account openness & Restrictions on capital account transactions & Prati, Onorato, and Papageorgiou (2013) \\
\hline Capital account openness, residents & Restrictions on financial credits \& personal capital transactions for residents & Prati, Onorato, and Papageorgiou (2013) \\
\hline Capital account openness, non-residents & Restrictions on financial credits for non-residents & Prati, Onorato, and Papageorgiou (2013) \\
\hline$\Delta$ FDI inflow (\% of GDP) & Foreign direct investment inflows ( $\%$ of GDP), 3-year change & UNCTAD \\
\hline Globalization index & KOF index of globalization ${ }^{43}$ & Dreher (2006), Dreher, Gaston, and Martens (2008) \\
\hline $\begin{array}{l}{ }^{42} \text { Indices range between } 0 \text { and } 1 \text {, high } \\
{ }^{43} \text { The three dimensions of the KOF in } \\
\text { trade, FDI, portfolio imvestment, and incor } \\
\text { trade and an index of capital controls. } \\
\text { international organizations to which the } \\
\text { (iii) Social globalization is expressed as th } \\
\text { tourism a country's population is exposed }\end{array}$ & $\begin{array}{l}\text { values indicate greater degree of liberalization. } \\
\text { ex are defined as follows: (i) Economic globalization refers to long distance flows of } \\
\text { payments to foreign nationals; and (b) Restrictions on trade and capital using hidd } \\
\text { Political globalization is characterized by a diffusion of government policies. It } \\
\text { untry is a member, (c) the number of UN peace missions it participated in, and ( } \\
\text { spread of ideas, information, images and people. It comprises (a) personal contacts } \\
\text { o, government and workers' transfers received and paid, the stock of foreign populat }\end{array}$ & $\begin{array}{l}\text { pital and services. It includes (a) Actual flows of } \\
\text { barriers, mean tariff rates, taxes on international } \\
\text { a) the number of embassies, (b) the number of } \\
\text { mber of international treaties it participates in. } \\
\text { ulude international telecom traffic, the degree of } \\
\text { umber of international letters sent and received; }\end{array}$ \\
\hline
\end{tabular}




\begin{tabular}{|c|c|c|}
\hline \multicolumn{3}{|c|}{ Table A3: Description and Source of Variables (Concluded) } \\
\hline Variable & Description & Source \\
\hline$\Delta$ FVA (\% exports) & Share of foreign value added exports in gross exports, 3-year change & Novta and Rodrigues Bastos (2016) \\
\hline$\Delta \mathrm{DVX}$ (\% exports) & $\begin{array}{l}\text { Share of domestic value added exports (excluding intermediary exports that } \\
\text { return home) in gross exports, 3-year change }\end{array}$ & Novta and Rodrigues Bastos (2016) \\
\hline$\Delta$ Term 3 (\% exports) & $\begin{array}{l}\text { Share of domestic value added embedded in intermediate goods exports } \\
\text { re-exported to third countries (in gross exports), 3-year change }\end{array}$ & Novta and Rodrigues Bastos (2016) \\
\hline$\Delta$ GVC participation & $\begin{array}{l}\text { Share of foreign value added exports and domestic value added exports } \\
\text { (excluding intermediary exports that return home) in gross exports, 3-year change }\end{array}$ & Calculated from Novta and Rodrigues Bastos (2016) \\
\hline Inflation & Inflation, consumer prices (annual \%) & WDI \\
\hline Human assets index & $\begin{array}{l}\text { Measure of human capital combining } 4 \text { indicators: (i) share of undernourished population; } \\
\text { ii) children mortality (aged } 5 \text { years or under); iii) gross secondary school enrollment rate; } \\
\text { iv) adult literacy rate }\end{array}$ & FERDI \\
\hline Population growth & Log difference of population & Calculated from WDI \\
\hline Government spending ( $\%$ of GDP) & General government final consumption expenditure (\% of GDP) & WDI \\
\hline Urban & Urban population (\% of total) & WDI \\
\hline Gender gap in labor force participation & Ratio of female to male labor force participation rate (\%) & WDI \\
\hline Unemployment & Unemployment (\% of total labor force) & WDI \\
\hline Gini index & Gini index of income inequality ${ }^{44}$ & WDI \\
\hline Crisis & $\begin{array}{l}\text { Dummy for the occurrence of a banking, currency and/or sovereign debt crisis, } \\
\text { including sovereign debt restructuring }\end{array}$ & Laeven and Valencia $(2008,2012)$ \\
\hline
\end{tabular}


Table A4: 5-year versus 9-year Horizon

\begin{tabular}{|c|c|c|c|c|c|c|c|c|}
\hline & \multicolumn{4}{|c|}{ 5-year horizon } & \multicolumn{4}{|c|}{ 9-year horizon } \\
\hline & \multicolumn{2}{|c|}{ Goods } & \multicolumn{2}{|c|}{ Services } & \multicolumn{2}{|c|}{ Goods } & \multicolumn{2}{|c|}{ Services } \\
\hline & World & LAC & World & $\mathrm{LAC}$ & World & $\mathrm{LAC}$ & World & LAC \\
\hline & \multicolumn{8}{|c|}{ Domestic Macroeconomic and Governance Indicators } \\
\hline$\Delta$ Investment & 0.003 & -0.029 & $0.048^{*}$ & $0.152^{* *}$ & -0.023 & 0.064 & -0.021 & $0.103^{*}$ \\
\hline REER volatility & $-0.001^{*}$ & $-0.001^{* *}$ & $-0.001^{*}$ & $-0.004^{* * *}$ & -0.000 & -0.000 & -0.001 & -0.001 \\
\hline$\Delta$ Infrastructure & 0.108 & -0.064 & -0.113 & $-0.353^{* *}$ & 0.076 & $0.235^{*}$ & 0.088 & -0.167 \\
\hline Quality of government & $0.033^{* *}$ & $0.139^{* * *}$ & $0.063^{* * *}$ & $0.120^{* * *}$ & -0.009 & $0.101^{* * *}$ & $0.053^{* * *}$ & $0.094^{* * *}$ \\
\hline Polity 2 & -0.001 & 0.007 & $0.007^{* * *}$ & 0.005 & 0.000 & $0.024^{* * *}$ & 0.002 & $0.015^{*}$ \\
\hline \multirow[t]{2}{*}{ Secondary education } & $0.002^{* * *}$ & $0.003^{*}$ & $0.001^{* *}$ & 0.000 & 0.000 & 0.000 & $0.002^{* * *}$ & 0.002 \\
\hline & \multicolumn{8}{|c|}{ Real Exchange Rate and Diversification } \\
\hline Log REER index & $-0.202^{* * *}$ & $-0.221^{* * *}$ & $-0.229^{* * *}$ & $-0.310^{* * *}$ & $-0.057^{* *}$ & $-0.139^{*}$ & -0.021 & -0.151 \\
\hline Log RER & $-0.113^{* * *}$ & $-0.241^{* * *}$ & $-0.059^{* *}$ & $-0.302^{* * *}$ & -0.006 & 0.021 & 0.002 & -0.041 \\
\hline RER misalignment & $-0.087^{* * *}$ & $-0.208^{* * *}$ & $-0.049^{*}$ & $-0.289^{* * *}$ & 0.006 & 0.030 & 0.002 & -0.041 \\
\hline Theil index & $-0.023^{* *}$ & $-0.111^{* * *}$ & $-0.061^{* * *}$ & $-0.174^{* * *}$ & 0.005 & -0.034 & -0.012 & -0.067 \\
\hline Theil, extensive margin & -0.004 & $-0.088^{*}$ & $-0.079^{* * *}$ & -0.016 & $0.041^{* * *}$ & -0.030 & $-0.042^{* *}$ & 0.009 \\
\hline \multirow{2}{*}{ Theil, intensive margin } & $-0.031^{* * *}$ & $-0.089^{* * *}$ & $-0.046^{* * *}$ & $-0.180^{* * *}$ & -0.013 & -0.025 & -0.002 & -0.087 \\
\hline & \multicolumn{8}{|c|}{ Trade Policy and Product Market Reforms } \\
\hline$\Delta$ Trade openness & 0.022 & $0.181^{* *}$ & 0.054 & $0.338^{* * *}$ & $0.063^{* * *}$ & -0.000 & 0.013 & 0.079 \\
\hline Tariffs & $-0.007^{* *}$ & 0.005 & $-0.010^{* * *}$ & $-0.044^{* * *}$ & -0.001 & -0.000 & -0.001 & -0.000 \\
\hline Tariffs, manufactures & $-0.007^{* *}$ & 0.008 & $-0.010^{* * *}$ & $-0.048^{* * *}$ & -0.001 & -0.000 & -0.001 & -0.000 \\
\hline Tariffs, primary products & -0.004 & -0.006 & $-0.006^{*}$ & 0.001 & -0.001 & -0.000 & -0.004 & -0.000 \\
\hline Agriculture index & 0.055 & 0.052 & $0.142^{* * *}$ & $0.270^{* * *}$ & $0.073^{* * *}$ & 0.028 & $0.050^{*}$ & $0.334^{* * *}$ \\
\hline \multirow[t]{2}{*}{ Electricity \& Telecoms } & $-0.256^{* * * *}$ & -0.248 & 0.107 & $0.353^{* *}$ & $-0.110^{* * *}$ & $-0.554^{* * * *}$ & $0.100^{* *}$ & $0.606^{* * *}$ \\
\hline & \multicolumn{8}{|c|}{ Financial Liberalization } \\
\hline Domestic financial liberalization & 0.114 & $-0.497^{*}$ & -0.067 & -0.247 & $-0.189^{* * *}$ & $-1.743^{* * *}$ & -0.133 & 0.349 \\
\hline Banking sector reform & 0.055 & $-0.461^{*}$ & -0.079 & -0.012 & $-0.166^{* *}$ & $-1.608^{* * *} *$ & -0.108 & 0.328 \\
\hline Securities market reform & $0.178^{* *}$ & -0.179 & 0.027 & $-0.602^{* * *} *$ & $-0.087^{*}$ & $-0.543^{* * *}$ & -0.077 & 0.062 \\
\hline Capital account openness & -0.082 & $0.535^{* * * *}$ & $0.138^{* *}$ & 0.228 & $-0.071^{*}$ & 0.048 & -0.038 & 0.234 \\
\hline Capital acc. openness, residents & -0.051 & $0.451^{* * *}$ & 0.076 & 0.144 & -0.031 & 0.182 & -0.035 & 0.065 \\
\hline \multirow[t]{2}{*}{ Cap. acc. openness, non-residents } & -0.083 & $0.439^{* * *}$ & $0.180^{* * * *}$ & $0.269^{*}$ & $-0.097^{* * *}$ & -0.139 & -0.004 & $0.411^{* *}$ \\
\hline & \multicolumn{8}{|c|}{ Globalization and GVC Participation } \\
\hline$\Delta$ FDI inflow (\% GDP) & $0.014^{* *}$ & 0.010 & -0.012 & $0.080^{* * * *}$ & $0.010^{* *}$ & 0.017 & -0.009 & 0.013 \\
\hline Globalization index & 0.001 & $0.012^{* * *}$ & $0.006^{* * *}$ & $0.008^{*}$ & $-0.002 * *$ & 0.004 & $0.003^{* * *}$ & $0.015^{* * *}$ \\
\hline$\Delta$ FVA (\% exports) & $0.192^{* * *}$ & $0.589^{* *}$ & $0.338^{* * * *}$ & $1.179^{* * *}$ & $0.056^{*}$ & $0.635^{* *}$ & 0.064 & 0.475 \\
\hline$\Delta$ DVX (\% exports) & -0.041 & 0.067 & $-0.240^{* *}$ & -0.472 & 0.106 & 0.439 & -0.106 & -0.149 \\
\hline$\Delta$ Term 3 (\% exports) & -0.064 & 0.108 & $-0.209^{* *}$ & -0.365 & 0.091 & $0.626^{*}$ & -0.095 & 0.100 \\
\hline$\Delta$ GVC participation & $0.436^{* * *}$ & 0.358 & $0.470^{* * *}$ & $1.359^{* * *}$ & $0.286^{* * *}$ & $1.351^{* * *}$ & $0.206^{*}$ & 0.795 \\
\hline
\end{tabular}


Table A5: One Tenth versus Twofold Increase in Export Growth

\begin{tabular}{|c|c|c|c|c|c|c|c|c|}
\hline & \multicolumn{4}{|c|}{ Criterion 2, 1.1} & \multicolumn{4}{|c|}{ Criterion $2,2.0$} \\
\hline & \multicolumn{2}{|c|}{ Goods } & \multicolumn{2}{|c|}{ Services } & \multicolumn{2}{|c|}{ Goods } & \multicolumn{2}{|c|}{ Services } \\
\hline & World & LAC & World & LAC & World & LAC & World & $\mathrm{LAC}$ \\
\hline & \multicolumn{8}{|c|}{ Domestic Macroeconomic and Governance Indicators } \\
\hline$\Delta$ Investment & 0.022 & 0.054 & 0.032 & $0.117^{\text {**** }}$ & 0.017 & 0.047 & 0.026 & $0.117^{* * *}$ \\
\hline REER volatility & $-0.001^{*}$ & $-0.001^{* *}$ & -0.000 & $-0.004^{* * *}$ & $-0.001^{*}$ & $-0.001^{* *}$ & -0.000 & $-0.004^{* * *}$ \\
\hline$\Delta$ Infrastructure & $0.139 * *$ & 0.121 & 0.038 & $-0.267^{* *}$ & 0.083 & 0.043 & 0.048 & $-0.267^{* *}$ \\
\hline Quality of government & $0.023^{* *}$ & $0.157^{* * *}$ & $0.042^{* * *}$ & $0.100^{* * *}$ & 0.007 & $0.152^{* * *}$ & $0.034^{* *}$ & $0.100^{* * *}$ \\
\hline Polity 2 & 0.000 & $0.024^{* * *}$ & 0.001 & -0.000 & -0.000 & $0.021^{* * *}$ & $0.002^{*}$ & -0.000 \\
\hline \multirow[t]{2}{*}{ Secondary education } & $0.002^{* * * *}$ & $0.007^{* * * *}$ & $0.001^{* * * *}$ & 0.001 & $0.001^{* * *}$ & $0.006^{* * *}$ & $0.001^{* * * *}$ & 0.001 \\
\hline & \multicolumn{8}{|c|}{ Real Exchange Rate and Diversification } \\
\hline Log REER index & $-0.138^{* * *}$ & $-0.317^{* * *}$ & $-0.121^{* * *}$ & $-0.211^{*}$ & $-0.122^{* * *}$ & $-0.327^{* * *}$ & $-0.134^{* * *}$ & $-0.211^{*}$ \\
\hline Log RER & $-0.073^{* * *}$ & $-0.166^{*}$ & $-0.061^{* *}$ & -0.089 & $-0.062^{* *}$ & $-0.176^{* *}$ & $-0.048^{*}$ & -0.089 \\
\hline RER misalignment & $-0.057^{* *}$ & $-0.128^{*}$ & $-0.052^{* *}$ & -0.077 & $-0.046^{* *}$ & $-0.136^{*}$ & -0.039 & -0.077 \\
\hline Theil index & $-0.025^{* * *}$ & -0.045 & $-0.025^{* *}$ & $-0.117^{* * *}$ & $-0.020 * *$ & -0.052 & $-0.024^{* *}$ & $-0.117^{* * *}$ \\
\hline Theil, extensive margin & -0.005 & $-0.140^{* *}$ & $-0.032^{*}$ & -0.057 & 0.002 & $-0.113^{*}$ & $-0.031^{*}$ & -0.057 \\
\hline \multirow[t]{2}{*}{ Theil, intensive margin } & $-0.032^{* * *}$ & 0.008 & $-0.020^{*}$ & $-0.113^{* * *}$ & $-0.028^{* * *}$ & -0.008 & -0.019 & $-0.113^{* * *}$ \\
\hline & \multicolumn{8}{|c|}{ Trade Policy and Product Market Reforms } \\
\hline$\Delta$ Trade openness & 0.045 & $0.252^{*}$ & 0.043 & $0.273^{* * *}$ & 0.035 & $0.277^{* *}$ & 0.041 & $0.273^{* * *}$ \\
\hline Tariffs & $-0.006^{* *}$ & -0.001 & -0.004 & $-0.020^{*}$ & -0.004 & 0.001 & -0.002 & $-0.020^{*}$ \\
\hline Tariffs, manufactures & $-0.006^{* *}$ & 0.003 & -0.004 & -0.018 & $-0.004^{*}$ & 0.003 & -0.001 & -0.018 \\
\hline Tariffs, primary products & -0.002 & -0.015 & -0.006 & $-0.023^{* * *}$ & -0.001 & -0.007 & -0.004 & $-0.023^{* * *}$ \\
\hline Agriculture index & $0.060^{* *}$ & $0.214^{* *}$ & $0.119^{* * *}$ & $0.296 * * *$ & $0.071^{* * *}$ & $0.177^{*}$ & $0.102 * * *$ & $0.296^{* * *}$ \\
\hline \multirow[t]{2}{*}{ Electricity \& Telecoms } & $-0.114^{* *}$ & 0.024 & $0.172^{* * * *}$ & $0.568^{* * *}$ & $-0.092^{* *}$ & 0.227 & $0.156^{* * * *}$ & $0.568^{* * * *}$ \\
\hline & \multicolumn{8}{|c|}{ Financial Liberalization } \\
\hline Domestic financial liberalization & -0.090 & -0.253 & -0.029 & $0.656^{* *}$ & -0.016 & 0.024 & -0.136 & $0.656^{* *}$ \\
\hline Banking sector reform & -0.105 & -0.257 & -0.019 & $0.720^{* * * *}$ & -0.009 & -0.041 & -0.102 & $0.720^{* * * *}$ \\
\hline Securities market reform & 0.030 & -0.011 & -0.031 & -0.021 & -0.020 & 0.121 & -0.103 & -0.021 \\
\hline Capital account openness & -0.063 & $0.454^{* * *}$ & 0.041 & 0.223 & -0.024 & $0.408^{* * *}$ & 0.017 & 0.223 \\
\hline Capital acc. openness, residents & -0.066 & $0.383^{* * *}$ & 0.024 & 0.107 & -0.030 & $0.351^{* * *}$ & -0.012 & 0.107 \\
\hline \multirow[t]{2}{*}{ Cap. acc. openness, non-residents } & -0.040 & $0.401^{* * *}$ & 0.064 & $0.322^{* *}$ & -0.006 & $0.350^{* *}$ & 0.058 & $0.322^{* *}$ \\
\hline & \multicolumn{8}{|c|}{ Globalization and GVC Participation } \\
\hline$\Delta$ FDI inflow (\% GDP) & $0.012^{* *}$ & 0.010 & -0.012 & $0.049^{*}$ & $0.014^{* * *}$ & 0.001 & -0.011 & $0.049^{*}$ \\
\hline Globalization index & 0.001 & $0.014^{* * *}$ & $0.003^{* *}$ & $0.009^{* * *}$ & -0.000 & $0.013^{* * *}$ & $0.003^{* * *}$ & $0.009^{* * *}$ \\
\hline$\Delta$ FVA (\% exports) & $0.083^{* *}$ & $0.513^{*}$ & $0.229^{* * *}$ & $1.048^{* * * *}$ & 0.046 & $0.695^{*}$ & $0.272^{* * *}$ & $1.048^{* * *}$ \\
\hline$\Delta$ DVX (\% exports) & 0.063 & $1.082^{* *}$ & $-0.230^{* *}$ & -0.040 & 0.099 & $1.106^{* *}$ & $-0.249^{* * *}$ & -0.040 \\
\hline$\Delta$ Term 3 (\% exports) & 0.044 & $1.155^{* * *}$ & $-0.213^{* *}$ & 0.037 & 0.096 & $1.209^{* * *}$ & $-0.226^{* * *}$ & 0.037 \\
\hline$\Delta$ GVC participation & $0.278^{* * *}$ & $1.821^{* * *}$ & $0.369^{* *}$ & $1.439^{* * *}$ & $0.179^{* *}$ & $1.990^{* * *}$ & $0.363^{* *}$ & $1.439^{* * *}$ \\
\hline \multicolumn{9}{|c|}{$\begin{array}{l}\text { Notes: Probit estimates. The dependent variable is a dummy for the timing of export accelerations which equals } 1 \text { over the } 3 \text {-year window } \\
\text { centered on the initiation date. Coefficients are marginal probabilities evaluated at the sample means. } * \text {, } * * \text { and } * * * \text { denote statistical } \\
\text { significance at the } 10 \%, 5 \% \text { and } 1 \% \text { level respectively. Variables are lagged by } 2 \text { years. All variables in the baseline model are included but } \\
\text { not reported. LAC stands for Latin America and the Caribbean. The description and source of variables are provided in Table A3. }\end{array}$} \\
\hline
\end{tabular}


Table A6: Export Acceleration Threshold of 2 percentage points versus 4 percentage points

\begin{tabular}{|c|c|c|c|c|c|c|c|c|}
\hline & \multicolumn{4}{|c|}{ Criterion 2,2 ppts } & \multicolumn{4}{|c|}{ Criterion 2, 4 ppts } \\
\hline & \multicolumn{2}{|c|}{ Goods } & \multicolumn{2}{|c|}{ Services } & \multicolumn{2}{|c|}{ Goods } & \multicolumn{2}{|c|}{ Services } \\
\hline & World & $\mathrm{LAC}$ & World & $\mathrm{LAC}$ & World & LAC & World & $\mathrm{LAC}$ \\
\hline & \multicolumn{8}{|c|}{ Domestic Macroeconomic and Governance Indicators } \\
\hline$\Delta$ Investment & 0.019 & 0.054 & 0.032 & $0.117^{* * * *}$ & 0.021 & 0.031 & 0.028 & $0.117^{* * * *}$ \\
\hline REER volatility & $-0.001^{*}$ & $-0.001^{* *}$ & -0.001 & $-0.004^{* * *}$ & $-0.001^{*}$ & $-0.001^{* *}$ & -0.000 & $-0.004^{* * *}$ \\
\hline$\Delta$ Infrastructure & $0.139^{* *}$ & 0.121 & 0.043 & $-0.206^{*}$ & $0.117^{*}$ & 0.038 & 0.056 & $-0.267^{* *}$ \\
\hline Quality of government & $0.025^{* *}$ & $0.157^{* * * *}$ & $0.049^{* * *}$ & $0.108^{* * *}$ & 0.017 & $0.177^{* * *}$ & $0.039^{* * *}$ & $0.100^{* * *}$ \\
\hline Polity 2 & 0.001 & $0.024^{* * *}$ & 0.002 & 0.001 & -0.001 & $0.022^{* * *}$ & 0.002 & -0.000 \\
\hline \multirow[t]{2}{*}{ Secondary education } & $0.002^{* * *}$ & $0.007^{* * * *}$ & $0.001^{* * * *}$ & 0.001 & $0.001^{* * *}$ & $0.007^{* * *}$ & $0.001^{* * *}$ & 0.001 \\
\hline & \multicolumn{8}{|c|}{ Real Exchange Rate and Diversification } \\
\hline Log REER index & $-0.149^{* * *}$ & $-0.317^{* * *}$ & $-0.125^{* * *}$ & $-0.236^{* *}$ & $-0.135^{* * *}$ & $-0.325^{* * *}$ & $-0.125^{* * *}$ & $-0.211^{*}$ \\
\hline Log RER & $-0.071^{* * *}$ & $-0.166^{*}$ & $-0.054^{* *}$ & -0.100 & $-0.073^{* * *}$ & -0.136 & $-0.055^{* *}$ & -0.089 \\
\hline RER misalignment & $-0.055^{* *}$ & $-0.128^{*}$ & $-0.046^{*}$ & -0.088 & $-0.057^{* *}$ & -0.101 & $-0.047^{*}$ & -0.077 \\
\hline Theil index & $-0.027^{* * *}$ & -0.045 & $-0.028 * * *$ & $-0.119^{* * *}$ & $-0.021^{* *}$ & -0.024 & $-0.027^{* * *}$ & $-0.117^{* * *}$ \\
\hline Theil, extensive margin & -0.007 & $-0.140^{* *}$ & $-0.035^{*}$ & $-0.081^{*}$ & -0.001 & -0.092 & $-0.041^{* *}$ & -0.057 \\
\hline \multirow[t]{2}{*}{ Theil, intensive margin } & $-0.033^{* * *}$ & 0.008 & $-0.022^{*}$ & $-0.103^{* *}$ & $-0.028^{* * *}$ & 0.012 & -0.018 & $-0.113^{* * *}$ \\
\hline & \multicolumn{8}{|c|}{ Trade Policy and Product Market Reforms } \\
\hline$\Delta$ Trade openness & 0.040 & $0.252^{*}$ & 0.043 & $0.285^{* * *}$ & 0.043 & $0.212^{*}$ & 0.044 & $0.273^{\text {*** }}$ \\
\hline Tariffs & $-0.007^{* *}$ & -0.001 & -0.005 & $-0.028^{* *}$ & -0.004 & 0.001 & -0.002 & $-0.020^{*}$ \\
\hline Tariffs, manufactures & $-0.007^{* *}$ & 0.003 & -0.004 & $-0.026^{* *}$ & -0.004 & 0.001 & -0.002 & -0.018 \\
\hline Tariffs, primary products & -0.004 & -0.015 & -0.006 & $-0.028^{* * *}$ & -0.001 & -0.001 & -0.004 & $-0.023^{* * *}$ \\
\hline Agriculture index & $0.069^{* * *}$ & $0.214^{* *}$ & $0.098^{* * *}$ & $0.328^{* * *}$ & $0.072^{* * *}$ & 0.135 & $0.098^{* * *}$ & $0.296^{* * *}$ \\
\hline \multirow[t]{2}{*}{ Electricity \& Telecoms } & $-0.157^{* * *}$ & 0.024 & $0.147^{* *}$ & $0.468^{* * * *}$ & $-0.129^{* * *} *$ & 0.157 & $0.153^{* * * *}$ & $0.568^{* * * *}$ \\
\hline & \multicolumn{8}{|c|}{ Financial Liberalization } \\
\hline Domestic financial liberalization & -0.100 & -0.253 & -0.153 & 0.440 & -0.086 & 0.019 & $-0.184^{*}$ & $0.656^{* *}$ \\
\hline Banking sector reform & -0.119 & -0.257 & -0.136 & $0.550^{* *}$ & -0.087 & -0.130 & $-0.160^{*}$ & $0.720^{* * *}$ \\
\hline Securities market reform & 0.038 & -0.011 & -0.061 & -0.144 & -0.008 & 0.279 & -0.080 & -0.021 \\
\hline Capital account openness & -0.070 & $0.454^{* * * *}$ & 0.044 & $0.283^{*}$ & $-0.101^{* *}$ & $0.286^{* *}$ & 0.000 & 0.223 \\
\hline Capital acc. openness, residents & $-0.068^{*}$ & $0.383^{* * *}$ & 0.009 & 0.152 & $-0.101^{* * *}$ & $0.255^{* *}$ & -0.026 & 0.107 \\
\hline \multirow[t]{2}{*}{ Cap. acc. openness, non-residents } & -0.056 & $0.401^{* * *}$ & 0.088 & $0.384^{* *}$ & $-0.073^{*}$ & $0.235^{*}$ & 0.048 & $0.322^{* *}$ \\
\hline & \multicolumn{8}{|c|}{ Globalization and GVC Participation } \\
\hline$\Delta$ FDI inflow (\% GDP) & $0.012^{* *}$ & 0.010 & $-0.013^{*}$ & $0.052^{*}$ & $0.014^{* * *}$ & 0.011 & -0.012 & $0.049^{*}$ \\
\hline Globalization index & -0.000 & $0.014 * * *$ & $0.003^{* * *}$ & $0.010^{* * *}$ & -0.000 & $0.011^{* * *}$ & $0.003^{* *}$ & $0.009^{* * *}$ \\
\hline$\Delta$ FVA (\% exports) & $0.081^{* *}$ & $0.513^{*}$ & $0.234^{* * *}$ & $1.089^{* * *}$ & $0.077^{* *}$ & 0.411 & $0.237^{* * *}$ & $1.048^{* * *}$ \\
\hline$\Delta$ DVX (\% exports) & 0.059 & $1.082^{* *}$ & $-0.232^{* *}$ & -0.085 & 0.042 & $0.779^{* *}$ & $-0.213^{* *}$ & -0.040 \\
\hline$\Delta$ Term 3 (\% exports) & 0.037 & $1.155^{* * *}$ & $-0.215^{* *}$ & -0.000 & 0.026 & $0.867^{* *}$ & $-0.201^{* *}$ & 0.037 \\
\hline$\Delta$ GVC participation & $0.277^{* * *}$ & $1.821^{* * *}$ & $0.383^{* *}$ & $1.412^{* *}$ & $0.254^{* * *}$ & $1.320^{* * *}$ & $0.374^{* *}$ & $1.439^{* * *}$ \\
\hline $\begin{array}{l}\text { Notes: Probit estimates. The depe } \\
\text { centered on the initiation date. } \\
\text { significance at the } 10 \%, 5 \% \text { and } 1 \text { \% } \\
\text { not reported. LAC stands for Lati }\end{array}$ & Ident varia & is a di & The & ng of exp & icceleration & which eql & 1 over the & vear windo \\
\hline
\end{tabular}




\begin{tabular}{|c|c|c|c|c|c|c|c|c|}
\hline \multicolumn{9}{|c|}{ Table A7: Standard Logit versus Rare Events Logit } \\
\hline & \multicolumn{4}{|c|}{$\begin{array}{ll}\text { Logit } \\
\end{array}$} & \multicolumn{4}{|c|}{ ReLogit } \\
\hline & \multicolumn{2}{|c|}{ Goods } & \multicolumn{2}{|c|}{ Services } & \multicolumn{2}{|c|}{ Goods } & \multicolumn{2}{|c|}{ Services } \\
\hline & World & LAC & World & LAC & World & LAC & World & LAC \\
\hline & \multicolumn{8}{|c|}{ Domestic Macroeconomic and Governance Indicators } \\
\hline$\Delta$ Investment & 0.024 & 0.042 & 0.033 & $0.109^{* * *}$ & -0.080 & -0.373 & 0.210 & $1.060^{* *}$ \\
\hline REER volatility & $-0.001^{*}$ & $-0.001^{*}$ & -0.000 & $-0.004^{* * *}$ & -0.006 & -0.002 & -0.007 & -0.057 \\
\hline D Infrastructure & $0.130^{* *}$ & 0.109 & 0.030 & $-0.218^{*}$ & $0.832^{*}$ & -0.162 & -0.361 & $-3.078^{* *}$ \\
\hline Quality of government & $0.024^{* *}$ & $0.160^{* * *}$ & $0.048^{* * *}$ & $0.092^{* * *}$ & 0.086 & $0.318^{*}$ & 0.059 & $0.442^{*}$ \\
\hline Polity 2 & 0.000 & $0.024^{* * *}$ & 0.001 & 0.000 & 0.002 & $0.081^{*}$ & $0.018^{*}$ & -0.021 \\
\hline Secondary education & $0.001^{* * *}$ & $0.006 * * *$ & $0.001^{* * *}$ & 0.001 & $0.009^{* * *}$ & $0.016^{* *}$ & $0.016^{* * * *}$ & $0.030^{* *}$ \\
\hline & \multicolumn{8}{|c|}{ Real Exchange Rate and Diversification } \\
\hline Log REER index & $-0.117^{* * *}$ & $-0.285^{* * *}$ & $-0.117^{* * *}$ & $-0.191^{*}$ & $-0.354^{*}$ & -0.398 & $-0.651^{* *}$ & -1.695 \\
\hline Log RER & $-0.077^{* * *}$ & -0.177 & $-0.056^{* *}$ & -0.072 & $-1.270^{* * *}$ & $-2.254^{* *}$ & $-0.537^{* * *}$ & -0.689 \\
\hline RER misalignment & $-0.064^{* * *}$ & -0.136 & $-0.049^{* *}$ & -0.063 & $-0.631^{* * *}$ & -1.138 & $-0.381^{* *}$ & -0.536 \\
\hline Theil index & $-0.025^{* * *}$ & -0.048 & $-0.025^{* * *}$ & $-0.109^{* * *}$ & $-0.195^{* * *}$ & -0.048 & $-0.164^{* *}$ & $-0.972^{*}$ \\
\hline Theil, extensive margin & -0.007 & $-0.132^{* *}$ & $-0.031^{*}$ & -0.046 & 0.029 & -0.169 & $-0.320^{* *}$ & -0.453 \\
\hline Theil, intensive margin & $-0.031^{* * *}$ & 0.000 & $-0.022^{*}$ & $-0.112^{* * *}$ & $-0.269^{* * *}$ & 0.021 & -0.085 & -0.782 \\
\hline \multicolumn{9}{|c|}{ Trade Policy and Product Market Reforms } \\
\hline$\Delta$ Trade openness & 0.036 & $0.231^{*}$ & 0.035 & $0.241^{\text {**** }}$ & 0.200 & $1.176^{*}$ & 0.234 & $1.472^{* *}$ \\
\hline Tariffs & $-0.005 * *$ & 0.000 & -0.006 & -0.017 & -0.015 & -0.058 & -0.030 & -0.158 \\
\hline Tariffs, manufactures & $-0.006^{* *}$ & 0.004 & -0.005 & -0.015 & -0.019 & -0.018 & -0.027 & -0.143 \\
\hline Tariffs, primary products & -0.002 & -0.013 & -0.007 & $-0.021^{* * *}$ & 0.009 & $-0.147^{* *}$ & $-0.036^{*}$ & $-0.276^{* *}$ \\
\hline Agriculture index & $0.050^{* *}$ & $0.210^{* *}$ & $0.085^{* * *}$ & $0.250^{* * *}$ & 0.223 & 0.056 & $0.757^{* * * *}$ & $2.216^{* * *}$ \\
\hline Electricity \& Telecoms & $-0.102^{\text {*** }}$ & 0.030 & $0.149^{* * * *}$ & $0.510^{* * * *}$ & -0.437 & $-1.801^{* * * *}$ & $2.250^{* * * *}$ & $1.732^{* * *}$ \\
\hline & \multicolumn{8}{|c|}{ Financial Liberalization } \\
\hline Domestic financial liberalization & -0.071 & -0.267 & -0.038 & $0.600^{* *}$ & $-1.104^{* * *}$ & $-3.116^{* * *}$ & $1.342^{* * *}$ & -0.357 \\
\hline Banking sector reform & -0.091 & -0.288 & -0.025 & $0.659^{* *}$ & $-1.108^{* * * *}$ & $-3.052^{* * * *}$ & $1.251^{* * * *}$ & -0.187 \\
\hline Securities market reform & 0.042 & 0.016 & -0.040 & -0.019 & -0.430 & $-1.716^{* * *}$ & 0.561 & -0.711 \\
\hline Capital account openness & -0.043 & $0.470^{\text {**** }}$ & 0.030 & 0.228 & $-0.618^{* * *}$ & -0.480 & $0.603^{*}$ & 0.500 \\
\hline Capital acc. openness, residents & -0.051 & $0.392^{* * *}$ & 0.001 & 0.101 & $-0.597^{* *}$ & -0.260 & 0.210 & 0.003 \\
\hline Cap. acc. openness, non-residents & -0.024 & $0.420^{* * *}$ & 0.073 & $0.332^{* *}$ & $-0.524^{*}$ & -0.637 & $0.886^{* * *}$ & 1.161 \\
\hline & \multicolumn{8}{|c|}{ Globalization and GVC Participation } \\
\hline$\Delta$ FDI inflow (\% GDP) & $0.012^{* *}$ & 0.006 & -0.012 & $0.045^{*}$ & -0.001 & -0.158 & $-0.125^{* * *}$ & 0.189 \\
\hline Globalization index & 0.001 & $0.013^{* * *}$ & $0.003^{* * *}$ & $0.008^{* *}$ & -0.001 & 0.002 & $0.046^{* * *}$ & $0.063^{* *}$ \\
\hline$\Delta$ FVA (\% exports) & $0.065^{* *}$ & $0.489^{*}$ & $0.235^{* * *}$ & $0.989^{* * * *}$ & 0.316 & $4.010^{* *}$ & $0.689^{* *}$ & $4.826^{* * *}$ \\
\hline$\Delta$ DVX (\% exports) & 0.070 & $1.030^{* *}$ & $-0.229^{* *}$ & -0.040 & -0.105 & $3.357^{*}$ & $-1.239^{* * *}$ & -0.434 \\
\hline$\Delta$ Term 3 (\% exports) & 0.048 & $1.062^{* * *}$ & $-0.212^{* *}$ & 0.038 & -0.196 & 2.369 & $-1.123^{* *}$ & -0.220 \\
\hline$\Delta$ GVC participation & $0.232^{* *}$ & $1.649^{* * *}$ & $0.367^{* *}$ & $1.299^{* *}$ & 0.765 & $10.685^{* * *}$ & 0.566 & $5.660^{* *}$ \\
\hline \multicolumn{9}{|c|}{$\begin{array}{l}\text { Notes: "Logit" refers to the standard logit regression; coefficients are marginal probabilities evaluated at the sample means. "ReLogit" is } \\
\text { the King and Zeng (2001)'s logit estimator corrected for rare occurrence bias; simple regression coefficients are reported instead of marginal } \\
\text { effects. The dependent variable is a dummy for the timing of export accelerations which equals } 1 \text { over the 3-year window centered on the } \\
\text { initiation date. *, ** and *** denote statistical significance at the } 10 \%, 5 \% \text { and } 1 \% \text { level respectively. Variables are lagged by } 2 \text { years. } \\
\text { All variables in the baseline model are included but not reported. LAC stands for Latin America and the Caribbean. The description and } \\
\text { source of variables are provided in Table A3. }\end{array}$} \\
\hline
\end{tabular}


Table A8: Disaggregating Exports versus Splitting the Sample Period

\begin{tabular}{|c|c|c|c|c|c|c|c|c|}
\hline & \multicolumn{4}{|c|}{ Disaggregated exports } & \multicolumn{4}{|c|}{ Period } \\
\hline & \multicolumn{8}{|c|}{ Domestic Macroeconomic and Governance Indicators } \\
\hline$\Delta$ Investment & 0.005 & $0.034^{* *}$ & 0.029 & $0.052^{* * *}$ & -0.010 & 0.051 & -0.022 & $0.097^{* * *}$ \\
\hline$\Delta$ Infrastructure & $0.200^{* * *}$ & $0.144^{* *}$ & -0.119 & -0.085 & 0.119 & 0.088 & -0.049 & 0.113 \\
\hline Quality of government & 0.016 & -0.008 & $0.045^{* * *}$ & $0.052^{* * * *}$ & -0.009 & $0.095^{* * * *}$ & $0.023^{*}$ & $0.114^{* * *}$ \\
\hline Polity 2 & -0.002 & 0.000 & $0.003^{* *}$ & $0.004^{* * *}$ & $0.003^{*}$ & $-0.005^{* *}$ & $0.003^{* *}$ & 0.000 \\
\hline Log REER index & $-0.075^{* *}$ & $-0.126^{* * *}$ & $-0.096^{* *}$ & $-0.059^{*}$ & $-0.108^{* * *}$ & -0.130 & $-0.080^{* * *}$ & -0.127 \\
\hline Log RER & $-0.064^{* * *}$ & -0.008 & -0.023 & $-0.046^{* *}$ & -0.019 & $-0.155^{* * *}$ & -0.030 & -0.086 \\
\hline RER misalignment & $-0.053^{* *}$ & -0.001 & -0.015 & $-0.048^{* *}$ & -0.010 & $-0.133^{* * *}$ & -0.022 & -0.078 \\
\hline Theil index & -0.002 & $-0.030^{* * *}$ & -0.010 & $-0.041 * * *$ & -0.014 & $-0.037^{* *}$ & -0.016 & $-0.049^{* *}$ \\
\hline Theil, extensive margin & $0.027^{*}$ & -0.014 & -0.004 & -0.017 & $0.038^{* *}$ & $-0.085^{* *}$ & $-0.031^{*}$ & -0.039 \\
\hline \multirow[t]{2}{*}{ Theil, intensive margin } & -0.017 & $-0.034^{* * *}$ & -0.012 & $-0.047^{* * *}$ & $-0.040^{* * *}$ & -0.020 & -0.006 & $-0.049^{* *}$ \\
\hline & \multicolumn{8}{|c|}{ Trade Policy and Product Market Reforms } \\
\hline \multirow[t]{2}{*}{ Electricity \& Telecoms } & $-0.157^{* * *}$ & 0.041 & 0.021 & 0.043 & -0.060 & $-0.124^{*}$ & $0.145^{*}$ & $0.193^{*}$ \\
\hline & \multicolumn{8}{|c|}{ Financial Liberalization } \\
\hline Domestic financial liberalization & -0.056 & -0.050 & 0.097 & 0.012 & 0.008 & $-0.365^{*}$ & -0.107 & $0.397^{*}$ \\
\hline Banking sector reform & -0.033 & -0.060 & 0.093 & 0.007 & -0.018 & $-0.331^{*}$ & -0.094 & 0.330 \\
\hline Securities market reform & -0.070 & 0.019 & 0.015 & 0.014 & 0.074 & -0.016 & -0.056 & 0.118 \\
\hline Capital account openness & -0.016 & 0.044 & 0.024 & -0.026 & $-0.096^{*}$ & 0.011 & -0.034 & $0.188^{*}$ \\
\hline Capital acc. openness, residents & -0.040 & 0.024 & 0.010 & -0.035 & $-0.084^{*}$ & -0.023 & -0.075 & $0.178 *$ \\
\hline \multirow[t]{2}{*}{ Cap. acc. openness, non-residents } & 0.005 & 0.031 & 0.038 & -0.025 & -0.080 & 0.058 & 0.030 & $0.187^{*}$ \\
\hline & \multicolumn{8}{|c|}{ Globalization and GVC Participation } \\
\hline$\Delta$ FDI inflow (\% GDP) & $0.018^{* * *}$ & $0.010^{* *}$ & $-0.017^{* *}$ & -0.004 & $0.015^{* *}$ & 0.008 & -0.008 & -0.019 \\
\hline Globalization index & 0.001 & $0.003^{* * *}$ & $0.004^{* * *}$ & $0.006^{* * *}$ & -0.001 & $0.004^{* * *}$ & -0.001 & $0.009^{* * *}$ \\
\hline$\Delta \mathrm{FVA}$ (\% exports) & $0.067 *$ & 0.050 & -0.053 & $0.141^{* * *}$ & 0.044 & $0.178^{* *}$ & $0.088^{* *}$ & $0.308^{* *}$ \\
\hline$\Delta$ DVX (\% exports) & 0.075 & 0.133 & 0.144 & -0.124 & -0.048 & 0.207 & $-0.096^{*}$ & $-0.278^{*}$ \\
\hline
\end{tabular}




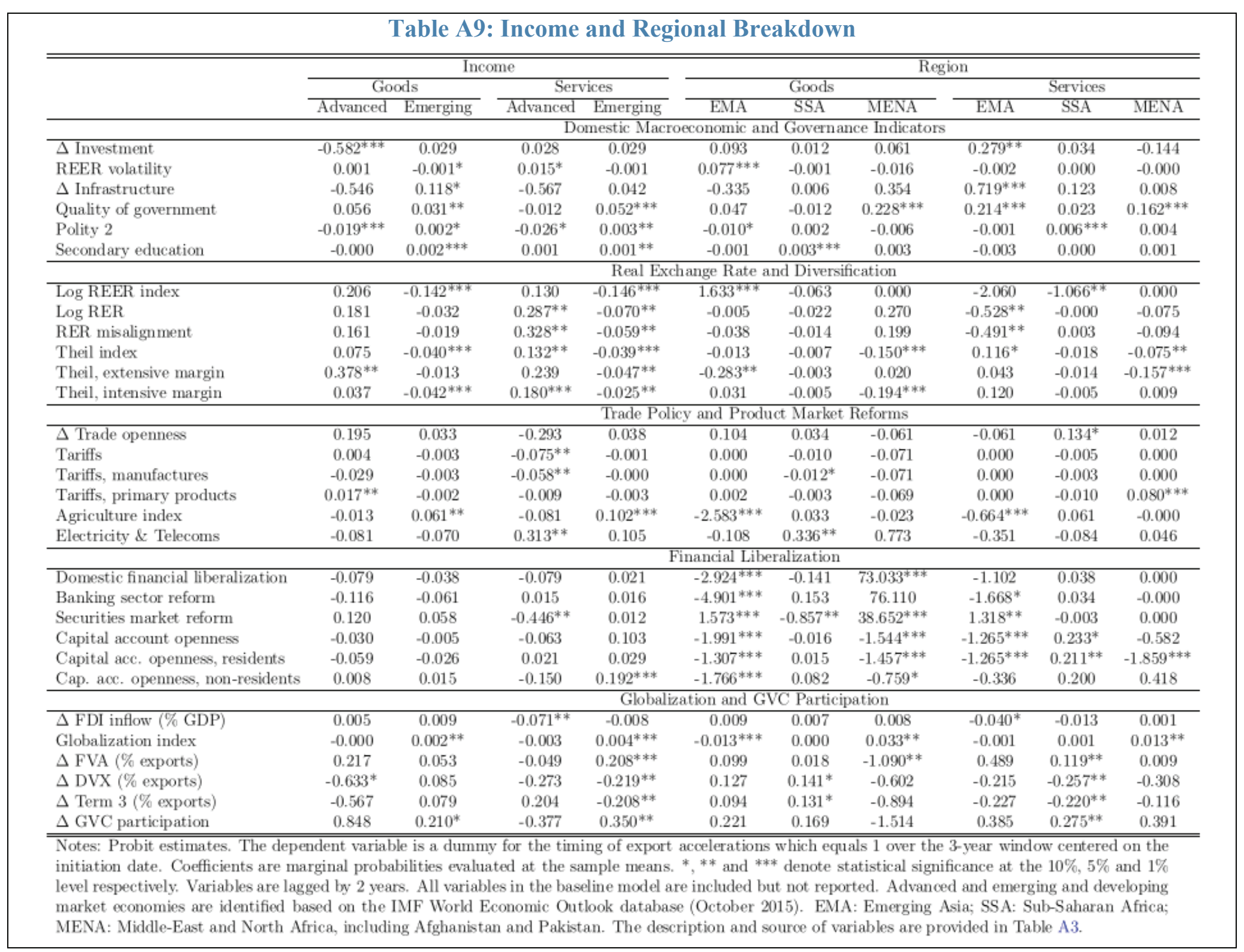


Table A10: Oil Dependence and Export Decelerations

\begin{tabular}{|c|c|c|c|c|c|c|c|}
\hline & \multicolumn{4}{|c|}{ Oil-dependence } & \multicolumn{3}{|c|}{ Export decelerations } \\
\hline & \multicolumn{2}{|c|}{ Goods } & \multicolumn{2}{|c|}{ Services } & \multicolumn{2}{|c|}{ Goods } & \multirow{2}{*}{$\begin{array}{c}\text { Services } \\
\text { World }\end{array}$} \\
\hline & Fuel & Non-fuel & Fuel & Non-fuel & World & LAC & \\
\hline & \multicolumn{7}{|c|}{ Domestic Macroeconomic and Governance Indicators } \\
\hline$\Delta$ Investment & 0.031 & 0.035 & 0.048 & 0.025 & $-0.682^{* *}$ & $-2.226^{* *}$ & $-1.008^{* * *}$ \\
\hline REER volatility & $-0.003^{*}$ & $-0.001^{* *}$ & $-0.004^{*}$ & -0.000 & $0.004^{* * *}$ & $0.041^{* * *}$ & $-0.175^{* * *}$ \\
\hline$\Delta$ Infrastructure & $0.722^{* * *}$ & 0.076 & $0.479^{* *}$ & 0.021 & -1.498 & $-3.598^{* * *}$ & 0.344 \\
\hline Quality of government & $0.194^{* * *}$ & 0.001 & $0.068^{* *}$ & $0.047^{* * *}$ & -0.344 & -1.100 & $-0.745^{* *}$ \\
\hline Polity 2 & $0.009^{* *}$ & 0.003 & -0.000 & 0.003 & $-0.062^{* *}$ & $-0.162^{* * *}$ & $-0.057^{*}$ \\
\hline \multirow[t]{2}{*}{ Secondary education } & $0.004^{* *}$ & $0.001^{* * *}$ & $0.003^{*}$ & 0.001 & 0.014 & 0.026 & $0.022^{*}$ \\
\hline & \multicolumn{7}{|c|}{ Real Exchange Rate and Diversification } \\
\hline Log REER index & $-0.717^{* * *}$ & $-0.169^{* * *}$ & $0.843^{* *}$ & $-0.319^{* * *}$ & $1.409^{* * *}$ & 1.486 & -0.457 \\
\hline Log RER & 0.103 & $-0.065^{* *}$ & 0.229 & $-0.123^{* * *}$ & -0.494 & 1.526 & $1.475^{* *}$ \\
\hline RER misalignment & 0.089 & -0.045 & 0.199 & $-0.099^{* * *}$ & -0.513 & $2.298^{* * *}$ & $1.273^{* *}$ \\
\hline Theil index & 0.001 & $-0.031^{* *}$ & $-0.138^{* * *}$ & $-0.032^{* *}$ & $0.769^{* * *}$ & $1.204^{*}$ & $-0.652^{* *}$ \\
\hline Theil, extensive margin & $0.101^{* *}$ & -0.027 & $-0.079^{*}$ & -0.027 & $0.765^{* *}$ & $1.640^{* * *}$ & 0.320 \\
\hline \multirow[t]{2}{*}{ Theil, intensive margin } & $-0.090^{* *}$ & -0.017 & -0.030 & -0.018 & 0.406 & 0.229 & $-0.846^{* * *}$ \\
\hline & \multicolumn{7}{|c|}{ Trade Policy and Product Market Reforms } \\
\hline$\Delta$ Trade openness & -0.063 & $0.113^{* * * *}$ & 0.033 & $0.115^{* *}$ & $-3.150^{* * *}$ & $-4.863^{* * *}$ & $-2.745^{* * *}$ \\
\hline Tariffs & $-0.030^{*}$ & -0.003 & $0.113^{* *}$ & -0.002 & $0.058^{* * *}$ & 0.076 & 0.035 \\
\hline Tariffs, manufactures & $-0.032 *$ & -0.003 & $0.117^{* *}$ & -0.001 & $0.056 * * *$ & 0.081 & $0.113^{* * *}$ \\
\hline Tariffs, primary products & $-0.023^{*}$ & -0.001 & $0.087^{* * *}$ & -0.003 & 0.035 & 0.009 & -0.039 \\
\hline Agriculture index & -0.076 & 0.052 & -0.008 & $0.110^{* * *}$ & 0.826 & -1.416 & 0.252 \\
\hline \multirow[t]{2}{*}{ Electricity \& Telecoms } & 0.256 & -0.093 & $-0.794^{*}$ & $0.174^{* *}$ & 1.599 & -0.872 & -0.892 \\
\hline & \multicolumn{7}{|c|}{$\begin{array}{l}\text { Financial Liberalization } \\
\end{array}$} \\
\hline Domestic financial liberalization & -0.389 & -0.078 & -0.000 & -0.023 & -0.712 & -0.378 & no obs. \\
\hline Banking sector reform & -0.587 & -0.076 & -0.000 & -0.007 & -0.891 & -1.059 & no obs. \\
\hline Securities market reform & 0.319 & 0.004 & $-0.054^{*}$ & -0.044 & 1.093 & $-5.218^{* *}$ & no obs. \\
\hline Capital account openness & 0.357 & -0.089 & -0.387 & 0.127 & $-5.099^{* * *}$ & -4.377 & no obs. \\
\hline Capital acc. openness, residents & 0.248 & -0.086 & -0.337 & 0.029 & $-3.517^{* * *}$ & $-2.632^{* *}$ & no obs. \\
\hline \multirow[t]{2}{*}{ Cap. acc. openness, non-residents } & 0.408 & -0.065 & -0.343 & $0.246^{* * *}$ & $-4.191^{* * *}$ & -5.798 & no obs. \\
\hline & \multicolumn{7}{|c|}{ Globalization and GVC Participation } \\
\hline$\Delta$ FDI inflow (\% GDP) & 0.004 & $0.016^{* * *}$ & $-0.085^{* *}$ & -0.010 & $-0.465^{* * *}$ & $-0.615^{* *}$ & $-0.240^{*}$ \\
\hline Globalization index & 0.006 & 0.000 & 0.005 & $0.004^{* *}$ & $-0.075^{* * *}$ & $-0.106^{* * *}$ & -0.015 \\
\hline$\Delta$ FVA (\% exports) & $-0.424^{* *}$ & $0.078^{*}$ & $0.923 * * *$ & $0.192^{* * *}$ & $-2.865 * * *$ & -4.310 & -3.455 \\
\hline$\Delta$ DVX (\% exports) & -0.685 & 0.117 & $-1.377^{*}$ & $-0.233^{* *}$ & $3.059^{* * *}$ & -4.301 & -0.023 \\
\hline$\Delta$ Term 3 (\% exports) & $-1.028^{*}$ & 0.113 & $-2.043^{* * *}$ & $-0.217^{* *}$ & $3.039^{* * *}$ & -2.873 & $3.543^{*}$ \\
\hline$\Delta$ GVC participation & $-1.812^{* * *}$ & $0.265^{* *}$ & 0.240 & $0.356^{* *}$ & 3.967 & -5.018 & -6.665 \\
\hline \multicolumn{8}{|c|}{$\begin{array}{l}\text { Notes: Probit estimates for "Oil-dependence", coefficients are marginal probabilities evaluated at the sample means. ReLogit } \\
\text { estimates for "Export decelerations", simple regression coefficients are reported instead of marginal effects. The dependent } \\
\text { variable is a dummy for the timing of export accelerations which equals } 1 \text { over the } 3 \text {-year window centered on the initiation } \\
\text { date. * }{ }^{* *} \text { and }{ }^{* * *} \text { denote statistical significance at the } 10 \%, 5 \% \text { and } 1 \% \text { level respectively. Variables are lagged by } 2 \text { years. All } \\
\text { variables in the baseline model are included but not reported. LAC stands for Latin America and the Caribbean. "Fuel" refers } \\
\text { to countries whose main source of export earnings (more than } 50 \% \text { of total exports on average between } 2010 \text { and 2014)) is fuel } \\
\text { (SITC section 3); "Non-fuel" pertains to SITC sections } 0,1,2,4 \text { and division } 68 \text { ), based on the IMF World Economic Outlook } \\
\text { database. "no obs.": no observations. The description and source of variables are provided in Table A3. }\end{array}$} \\
\hline
\end{tabular}

